\title{
Event-driven MAC Protocol For Dual-Radio Cooperation
}

\section{Arash Khatibi}





\title{
Event-driven MAC Protocol For Dual-Radio Cooperation
}

\author{
Master of Science Thesis
}

For the degree of Master of Science in Wireless and Mobile Communications Group (WMC)

at Department of Telecommunications at Delft University of Technology

Arash Khatibi

June 20, 2011

Faculty of Electrical Engineering, Mathematics and Computer Science Delft University of Technology Delft, The Netherlands

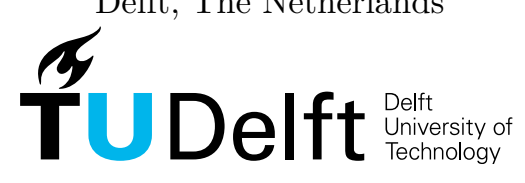


The work in this thesis was supported by the TRANS CCNP project. Their cooperation is hereby gratefully acknowledged.

\section{TंUDelft}

All rights reserved.

Copyright (c) Telecommunications Department

Faculty of Electrical Engineering, Mathematics and Computer Science

Delft University of Technology

Delft, The Netherlands 


\section{Delft University of TeChNology \\ DEPARTMENT OF \\ TELECOMMUNICATIONS}

The undersigned hereby certify that they have read and recommend to the Faculty of Electrical Engineering, Mathematics and Computer Science for acceptance a thesis entitled

\section{Event-driven MAC Protocol For Dual-Radio Cooperation}

by

ARASH KHATIBI

in partial fulfillment of the requirements for the degree of

MASTER OF SCIENCE.

Dated: June 20, 2011

Supervisor:

dr.E.Onur

Readers:

Prof.dr.ir.I.Niemegeers

dr.M.Simeoni

Y.Durmus 



\section{Abstract}

Energy efficiency is a critical issue in wireless sensor networks since nodes are battery-enabled devices. This issue seems more important in applications in which exchanging batteries costs a lot of energy, time, and money. A significant source of energy waste in wireless networks is due to the time that a node monitors the free channel. The time that a node listens to the channel while there is no packet transmission and the channel is free is called idle listening time. Using a low-power wake up radio is a great idea to decrease the energy consumption of wireless nodes in idle listening.

Dual-radio node model makes use of a wake up radio for channel monitoring and event detection, and a main radio for data exchange. The wake up radio's power consumption is much less than the main radio's. The wake up radio is always in monitoring state, listening to the channel. Since it consumes much less power in listening mode than the main radio, the high energy consumption of the wireless node during idle listening is decreased significantly. The main radio is in sleep state by default, and is woken up only in case the wake up radio detects an event (there is data to send or receive by the main radio). This method results in high energy efficiency, especially in applications with low event rates where the channel is idle most of the time.

In this project, we investigate the performance of the dual-radio node in wireless sensor networks. We design a new Medium Access Control (MAC) protocol for the dual-radio model to maximize the energy efficiency of the wireless network. We propose an analytical model to compute the energy consumption of a wireless node. Then, we implement the node model in OPtimized Network Evaluation Tool (OPNET) to test our model and validate our calculations. We model a wireless network equipped with dual-radio nodes and compare it with a Zigbee network in terms of energy consumption and delay.

When an event is detected by the wake up radio, it should wake the main radio up. This action takes time and consumes energy. For large event rates, this degrades the dual-radio performance. We define the Critical Event Rate as the rate above which 
the single-radio node model performs better than the dual-radio. The critical event rate depends on many parameters such as the characteristics of radio model and MAC protocol. We compute the critical event rate analytically and by simulation for a few radio models and clarify the method to be used for different models and MAC protocols.

We compare the energy consumption and delay of the dual-radio model with the single-radio Zigbee node for three radio types. We find the critical event rate and show how much energy is saved by using a wake up radio for different event rates. Finally, we propose the optimized MAC protocol based on the achieved results. The Rate-Adaptive MAC Protocol uses the wake up radio for event rates less than the critical event rate, and switches to the single-radio working method when the event rate passes the critical threshold. The contribution of our work is the new node model and MAC protocol and the rate-adaptive MAC proposal which works based on the critical event rate concept. 


\section{Table of Contents}

$\begin{array}{ll}\text { Acknowledgements } & \text { ix }\end{array}$

1 Introduction $\quad 1$

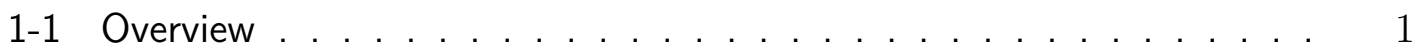

$1-2$ Rendezvous. . . . . . . . . . . . . . . . . . . . . . . . . . . . . . . 2

1-3 Wake-up Radio . . . . . . . . . . . . . . . . . . . . . . . . . . . . . 4 4

1-3-1 Advantages.................... 5

1-3-2 Challenges . . . . . . . . . . . . . . . . 6

1-3-3 Trade-offs . . . . . . . . . . . . . . . . . . . 6

1-4 Problem Definition . . . . . . . . . . . . . . . . . . . . . . . . . 7

1-5 Novelty and Contribution . . . . . . . . . . . . . . . 8

$1-6$ Structure of the Report . . . . . . . . . . . . . . . . . . . . . . . . . 8

2 Literature Review 9

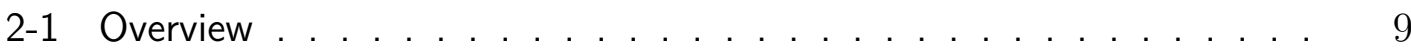

2-2 MAC Protocols . . . . . . . . . . . . . . . . . . . . . . . 9

2-2-1 Traditional MAC Families . . . . . . . . . . . . . . . 10

2-2-2 Scheduled Protocols . . . . . . . . . . . . . . . . . . . . . . . 12

2-2-3 Protocols With Common Active Periods . . . . . . . . . . . . 14

2-2-4 Preamble Sampling Protocols . . . . . . . . . . . . . . . . . . 15

2-2-5 Hybrid Protocols . . . . . . . . . . . . . . . . . . . . . . . . 15

2-2-6 Wake-up Radio MAC . . . . . . . . . . . . . . . . 16

2-3 Applications of Wireless Sensor Networks . . . . . . . . . . . . . . . . 17

2-4 Applications Description . . . . . . . . . . . . . . . . . . . . . 18

2-4-1 Intelligent Street Lightening . . . . . . . . . . . . . . . . . . . 18

2-4-2 Forest Fire Detection . . . . . . . . . . . . . . . . . . . . . . 19

2-5 MAC and Physical Layer Specifications . . . . . . . . . . . . . . . . . 21 
3 Analytical Model $\quad 25$

3-1 Overview . . . . . . . . . . . . . . . . . . . . 25

3-2 Analytical Model For Node Energy Consumption . . . . . . . . . . . . 25

3-2-1 SMAC Node Energy Consumption . . . . . . . . . . . . . . . . 26

3-2-2 Zigbee Node Energy Consumption . . . . . . . . . . . . . . . . 28

3-2-3 Range-based Wake-up Radio . . . . . . . . . . . . . . . . . . 29

3-2-4 Identity-based Wake-up Radio . . . . . . . . . . . . . . . . . . 31

3-3 Demonstration . . . . . . . . . . . . . . . . . . . . . . . . . . 32

4 Node Model Description and Results 37

4-1 Overview . . . . . . . . . . . . . . . . . . . . . . . . 37

4-2 Node Model . . . . . . . . . . . . . . . . . . . . . . . . 37

4-2-1 Network Allocation Vector . . . . . . . . . . . . . . . . . . . . 43

4-2-2 Busy Mode . . . . . . . . . . . . . . . . . . . . . . . . 43

4-2-3 Timers . . . . . . . . . . . . . . . . . . . 43

4-3 Results . . . . . . . . . . . . . . . . . . . . . . . . . . . 44

4-3-1 Energy Consumption . . . . . . . . . . . . . . . . . . . . . . 44

4-3-2 Delay . . . . . . . . . . . . . . . . . . . 56

4-3-3 Zigbee Beacon-enabled Network . . . . . . . . . . . . 57

4-3-4 Discussion . . . . . . . . . . . . . . . . . . . . . . . . 62

4-3-5 Advantages and Disadvantages . . . . . . . . . . . . . 64

4-3-6 Wake-up Relay . . . . . . . . . . . . . . . . . . . . . . . . . 65

4-4 Dual-radio Cooperation . . . . . . . . . . . . . . . . . . . . 66

5 Conclusion and Future Work $\quad 69$

5-1 Conclusion . . . . . . . . . . . . . . . . . . . . . . . . . . . . . . . 69

$5-2$ Future Work . . . . . . . . . . . . . . . . . . . . . . 70

$\begin{array}{ll}\text { Bibliography } & 73\end{array}$ 


\section{List of Figures}

1-1 Transmitter Initiated CyclEd Receivers (TICER) scheme . . . . . . . . 3

1-2 Receiver Initiated CyclEd Receivers (RICER) scheme . . . . . . . . . . 4

1-3 Dual-radio system . . . . . . . . . . . . . . . . . . . . . . . . . 5 5

$2-1$ CSMA/CA algorithm . . . . . . . . . . . . . . . . . . 11

3-1 SMAC protocol . . . . . . . . . . . . . . . . . 26

3-2 Zigbee non-beacon-enabled block diagram . . . . . . . . . . . . . . . 28

3-3 E2RMAC protocol . . . . . . . . . . . . . . . . . . . . . . . 30

3-4 Identity-based wake up MAC . . . . . . . . . . . . . . . . . . . 32

3-5 Analytical result for node energy consumption (Chipcon cc1000) . . . . 34

3-6 Analytical result for node energy consumption (LP Radio) . . . . . . . 35

3-7 Analytical result for node energy consumption (IMEC) . . . . . . . . . 35

4-1 Network model . . . . . . . . . . . . . . . . . . . . . . . . . 38

4-2 Zigbee node model . . . . . . . . . . . . . . . . . . . . . . . . . . . 39

4-3 Dual-radio node model . . . . . . . . . . . . . . . . . . . . . . . . . . 39

4-4 Dual-radio MAC block diagram . . . . . . . . . . . . . . . . . . . . . 40

4-5 CSMA/CA process model . . . . . . . . . . . . . . . . . . . . . 41

4-6 Zigbee MAC process model . . . . . . . . . . . . . . . . . . . . . . . 42

4-7 Zigbee node energy consumption (Chipcon cc1000) . . . . . . . . . . 45

4-8 Dual-radio node energy consumption (Chipcon cc1000) . . . . . . . . 45

4-9 Zigbee node energy consumption (LP Radio) . . . . . . . . . . . . . . 46

4-10 Dual-radio node energy consumption (LP Radio) . . . . . . . . . . . . 47

4-11 Zigbee node energy consumption (IMEC) . . . . . . . . . . . . . . . . 47 
4-12 Dual-radio node energy consumption (IMEC) . . . . . . . . . . . . . . 48

4-13 Dual-radio node energy consumption for $250 \mu \mathrm{J}$ switching energy (Chipcon cc1000)

4-14 Dual-radio node energy consumption for $550 \mu \mathrm{J}$ switching energy (Chipcon cc1000) . . . . . . . . . . . . . . . .

4-15 Dual-radio node energy consumption for $250 \mu \mathrm{J}$ switching energy (LP Radio) . . . . . . . . . . . . . . . . . . . .

4-16 Dual-radio node energy consumption for $550 \mu J$ switching energy (LP Radio)

4-17 Dual-radio node energy consumption for $150 \mu J$ switching energy (IMEC) 51

4-18 Dual-radio node energy consumption for $250 \mu \mathrm{J}$ switching energy (IMEC) 52

4-19 Dual-radio node energy consumption for $350 \mu J$ switching energy (IMEC) 52

4-20 Dual-radio node energy consumption for $450 \mu \mathrm{J}$ switching energy (IMEC) 53

4-21 Dual-radio node energy consumption for $550 \mu \mathrm{J}$ switching energy (IMEC) 53

4-22 Node energy consumption for different Transition Energies using IMEC (simulation) . . . . . . . . . . . . . . . . 54

4-23 Node energy consumption for different Transition Energies using IMEC (analytical) . . . . . . . . . . . . . . . . 55

4-24 Critical event rate . . . . . . . . . . . . . . . . . . . . . 55

4-25 Medium access delay . . . . . . . . . . . . . . . . . . . . . 56

4-26 End-to-end delay . . . . . . . . . . . . . . . . . . . . 57

4-27 Zigbee beacon-enabled node model . . . . . . . . . . . . . . . . . . 58

4-28 Superframe structure . . . . . . . . . . . . . . . . . . . . . . . . . . . 59

4-29 Zigbee beacon-enabled MAC process model . . . . . . . . . . . . . . . 59

4-30 Node energy consumption for different Transition Energies . . . . . . . 60

4-31 Node energy consumption for different duty cycles (Chipcon cc1000) . 61

4-32 Node energy consumption for different duty cycles (LP Radio) . . . . . 61

4-33 Node energy consumption for different duty cycles (IMEC) . . . . . . . 62 


\section{List of Tables}

3-1 Chipcon cc1000 . . . . . . . . . . . . . . . . . . . . . . . . 33

3-2 LP Radio . . . . . . . . . . . . . . . . . . . . . . . . . . . . . . . 33

3-3 IMEC . . . . . . . . . . . . . . . . . . . . . . . . . . 33

3-4 Demonstration parameters . . . . . . . . . . . . . . . . . . . . 34 


\section{Acknowledgements}

I would like to thank my mother and father for their supports, and Dr. Onur and

Yunus Durmus for their assistance during the writing of this thesis... 


\section{Chapter 1}

\section{Introduction}

\section{1-1 Overview}

Nodes in a wireless sensor network are battery-enabled devices and are not permanently connected to a power source. Since replacing sensors or batteries in many applications is not a cost-efficient task, minimizing energy consumption of wireless sensor networks is of significant importance. The factors that impact the energy consumption of sensors can be categorized by the OSI layers [1]. Physical layer parameters which affect the energy consumption of sensors are the technology employed such as narrowband or spread spectrum, method of increasing robustness to interference, and the design decisions about the trade-off between bandwidth efficiency and power consumption. Factors related to data link layer include overhearing, idle listening, and retransmissions due to collisions. Point-to-point reliability solutions like multipath routing, messages sent for route establishment, path augmentation (not using the shortest path to sink due to the use of energy-aware routing methods or localized protocols), and idle listening caused by unnecessary wake-ups are the network layer factors that have influence on energy efficiency. Messaging done to provide end-to-end reliability, flow control, and congestion avoidance are the transport layer factors. Finally, considering application layer, packet redundancy such as correlated event detections, messaging caused by application requirements such as software updates, and messaging for monitoring activities like health checking of sensors are categorized in this group.

In order to increase the power efficiency of a wireless sensor network, we should study the energy waste sources and find out methods to minimize them. We focus on the data link layer factors that impact the energy efficiency of the wireless sensors in this project. Overhearing, idle listening, and retransmissions due to collisions are the main sources of energy waste which are categorized as the data link layer factors. We try to propose the best method to minimize the energy wasted due to these causes. 
In this chapter, we talk about basic concepts and definitions about medium access control and describe some issues related to wake up radio. Then, we define the problem and introduce our solutions and the novelty of our work. Lastly, the structure of the next chapters of the report is explained.

The next section explains the schemes of how to make a node aware of when data is sent to it, which is referred to as Rendezvous.

\section{1-2 Rendezvous}

When two nodes in a wireless network intend to communicate with each other, the receiver should be awake when the sender starts to transmit data. This is referred to as rendezvous [1]. There are three types of rendezvous schemes:

\section{Pure synchronous:}

In this type, nodes are pre-synchronized so that their wake up time is known. This means that each node knows the active time of the other nodes to transmit data to them. One drawback of this scheme is that time synchronization results in considerable energy consumption. One other disadvantage of this category is that nodes wake up even when there is no data to transmit or receive which increases the idle listening time.

2. Pseudo-asynchronous:

Node which intends to transmit data, emits a preamble signal that indicates the destination node. The duration of preamble is long enough to coincide with the wake up time of the destination. High energy consumption for preamble sending, and obligation to follow a duty cycle are the disadvantages of this group of rendezvous schemes.

3. Pure asynchronous:

Nodes wake up on demand by the use of very low-power wake up radios. When a node has data to send, it first wakes up the main radio of the destination by sending a wake up request message by the wake up radio, and then sends data by the main radio. This group is the focus of this project.

Since a large proportion of the total energy consumption of wireless sensor nodes is due to idle listening, one of the best methods to decrease the energy consumption is to reduce this period. It is vital that the receiver of a message be awake when the transmitter wants to send data and this should be feasible without extending the active time of the node too much. Therefore, choosing the best rendezvous scheme is one of the most important keys of designing an energy-efficient wireless network.

There are different rendezvous methods for wireless sensor networks. In sleep scheduling scheme, nodes are powered on and off periodically and a beaconing approach is used to express the desire to communicate [2]. Depending on who initiates rendezvous, cycled schemes can be classified into two groups: Transmitter Initiated CyclEd Receivers (TICER) and Receiver Initiated CyclEd Receivers (RICER). 


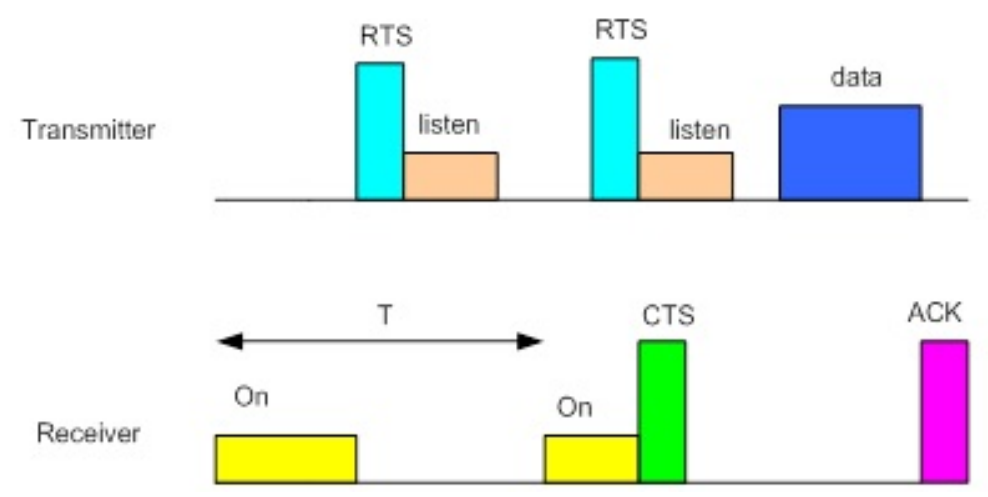

Figure 1-1: Transmitter Initiated CyclEd Receivers (TICER) scheme: In TICER, the transmitter initiates rendezvous. A node that has data to send, sends Request To Send after monitoring the channel for $O n$ duration, and waits for Clear To Send during listen period. After transmission of the data packet, the session ends with an Acknowledgement.

TICER scheme is shown in Figure 1-1. In TICER, when a node has no data to transmit, it wakes up to monitor the channel with a specific period and goes back to sleep after a wake up duration $T_{o n}$. As soon as a sensor node has data packets for transmission, it wakes up and monitors the channel for $T_{o n}$. If it does not hear anything, it starts transmitting Request To Send (RTS) and monitors the channel for a time $T_{l}$ for response. In Figure 1-1, $T_{\text {on }}$ and $T_{l}$ are shown as $O n$ and listen and $T$ is the length of one period. After reception of Clear To Send (CTS), the source node transmits the data packet and the session ends with an Acknowledgement (ACK).

Figure 1-2 shows the RICER scheme. In RICER, a node without data to transmit wakes up periodically to sense the channel. It sends a wake up beacon to announce that it is awake. If there is no response, it goes back to sleep. A source node monitors the channel for wake up beacons from the destination. Transmission starts upon the reception of the wake up beacon. Again the session ends with an ACK. Substantial power is saved if the wake up duration is set to it's optimal value. It can be shown that if there is no possibility to adjust the wake up period adaptively, it would be better to assume a heavy traffic load [2]. It can also be shown that network protocols that enable routing through multiple paths are very desirable to achieve higher energy efficiency [2].

Topology management schemes coordinate which nodes turn their radio off and when, such that the traffic forwarding remains satisfactory while minimizing the network energy consumption [3]. Sparse Topology and Energy Management (STEM) allows to efficiently trade one design constraint (energy) for the other (latency). It reduces the energy consumption in the monitoring state while ensuring satisfactory latency for transitioning to the transfer state [4].

In the default mode, the node's sensors and some preprocessing circuitry are the only active components of it. The main processor is only awakened when an event is detected. To forward the data to the final destination, nodes on the path should be awakened. They may not be able to sense the event. Thus, there may be in their inactive mode. One idea to solve this problem is that each node turns it's 


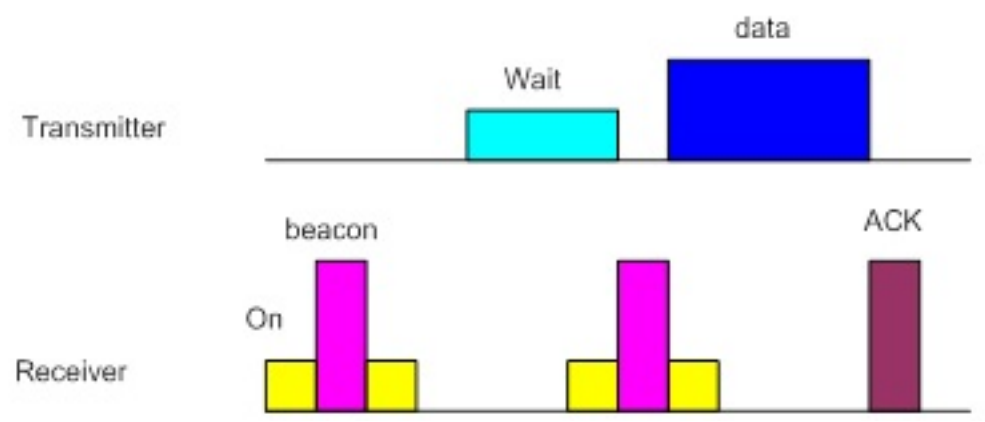

Figure 1-2: Receiver Initiated CyclEd Receivers (RICER) scheme: In RICER, the receiver is the initiator of rendezvous. A node with data for a sepecific target should wait for the wake up beacon of the destination. When it receives the beacon, the transmission session starts.

main radio on periodically to see if there are any packets destined for it. The node that intends to send data for a target, polls the target node in order to start data transmission. Once the link between nodes is activated, data is transmitted using a MAC protocol.

STEM-B (beacon) and STEM-T (tone) are the two categories of STEM schemes. STEM-B sends a beacon that includes the identity of the sender and destination while STEM-T sends a busy tone that wakes every node that hears it.

There is a trade-off between energy consumption and delay in wireless networks. If the active period of a node is decreased, it's energy consumption will decrease, but the delay in data exchange is increased since nodes should wait for a long time for the active period of their destinations. Rendezvous schemes try to find the optimum point which achieves proper energy efficiency and delay. The idea of using a lowpower wake up radio has the same goal. It intends to minimize the energy waste due to idle listening while achieving proper delay. The next section talks about the wake up radio.

\section{1-3 Wake-up Radio}

One method to decrease the energy consumption of nodes, while conveying data to destination with small delay, is to implement two radios on each node: one radio as the main radio for data transmission and reception, and a low-power wake up radio for monitoring the channel and event detection. The control channel is implemented using a low-power radio which can either operate on a frequency band other than the one used by the data channel or use the same channel [5]. The wake up radio does not need a complex MAC or sophisticated front end. It just monitors the channel and when receives a wake up tone from other wake up radios, or detects an event to report, switches the main radio to active mode for data reception or transmission. When a node has data to send, it sends a wake up tone by it's wake up radio and then transmits the data packet through the main channel. Although this scheme has higher implementation costs, due to significant reduction in energy consumption, it results in reduction in overall costs, and achieves a good balance between energy 


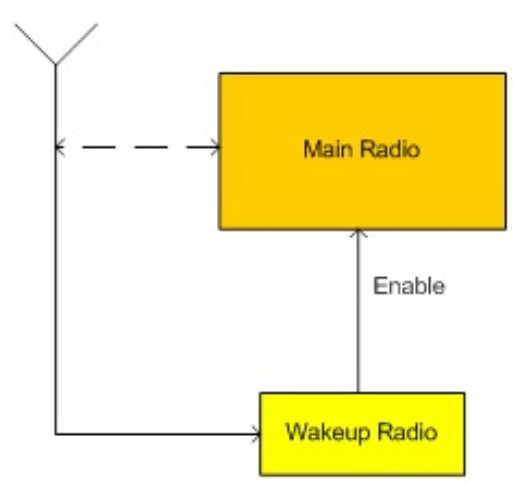

Figure 1-3: Dual-radio system: The wake up radio monitors the channel and enables the main radio when an event is detected. Data transmission is done by the main radio.

cosumption and delay. Figure 1-3 depicts a simple model of dual-radio system [6].

Wake up receivers can be classified from different points of view:

- Energy source: Based on energy source, wake up receivers may be passive or active. In passive wake up receivers, an external energy source triggers the wake up circuitry, while in active receivers the internal battery is used.

- Wake up signal: Based on wake up signal type, they may be radio-based or acoustic. In radio-based, radio signals are used as wake up signals. In contrast, the acoustic wake up receivers work with acoustic signals (external sounds).

- Wake up channel: According to wake up channel, they are categorized to shared or separate. The first type, uses the same channel for main radio and wake up radio, while the second group uses separate channels for the two radios.

- Destination specification: Regarding destination specification, they are classified to identity-based and range-based [1]. In identity-based wake up radios, the destination address is mentioned in the wake up tone. Hence, only the target node switches it's main radio to active state. The wake up tone of the range-based wake up radios does not contain the target address and all the nodes that are within the range of the wake up radio, switch their main radio to active mode once they receive the tone.

The advantages, challenges, and trade-offs of using a wake up radio are discussed below.

\section{1-3-1 Advantages}

Using wake up radio in wireless sensor networks results in energy efficiency, less overhead and overhearing, dynamic behavior, and different forms of use [1], [7].

- Energy conservation: It is a result of reduced idle listening of the main radio which is an important source of enegy consumption. 
- Less overhead: The wake up receiver presents a purely asynchronous rendezvous scheme so that the overhead caused by time synchronization which is a source of energy waste is decreased. The communication overhead for routing is also decreased because each node wakes up it's neighbors.

- Less overhearing: With the help of identity-based wake up receivers, only the destination node is woken up so that overhearing is decreased.

- Dynamic behavior: Since the duty cycle is dynamic, several inefficient parameters like preamble length should be dynamically set and become unnecessary.

- Different forms of use: Finally, the wake up receiver technology enables various new methods like the on-demand target monitoring [8].

\section{1-3-2 Challenges}

There are some challenges in using wake up receivers which should be studied in order to take advantage of the mentioned benefits [1]:

- Cost and hardware complexity: Utilizing separate channels results in more complex hardware and increased cost of nodes.

- Delay: Wake up delay may be high, especially end-to-end delay may become significant when number of hops is large.

- Topology change: Since the range of wake up radio is less than the main radio, topology for wake up receivers may be different from the actual network topology.

- Energy hole problem: Coverage hole is defined as the area not covered by any sensors due to anomalies such as node failures or jamming. If the wake up range is smaller than the communication range of the main radio, the topology of the network formed by the wake up receivers may contain more or larger energy holes.

- Increase of overhearing for shared channels and range-based wake up receivers: For range-based wake up receivers, in which all nodes that hear the wake up tone switch their main radio to active state, overhearing is increased. The same problem happens for the case of shared channel wake up receivers.

\section{1-3-3 Trade-offs}

Now we discuss some trade-offs in using wake up radios:

- Wake up range versus energy consumption: The range of a wake up radio can be adjusted with the energy consumption trade-off. Distant sensors can be awakened by consuming more energy. Determining an efficient wake up distance depends on design decisions such as the applied routing strategy. 
- Wake up range versus delay: End-to-end delay is a critical metric in wireless sensor networks since multihop communication is common. Increasing wake up range decreases the overall hop count and the end-to-end delay.

- In-band wake up radio versus out-of-band wake up radio: In in-band wake up radio the same channel is used for wake up and data transmission while in outof-band wake up radio separate channels are used for these purposes. Using the same channel for wake up and data transmission reduces the implementation costs, but has lower resistance to jamming and higher probability of collision and larger delay.

\section{1-4 Problem Definition}

There are different solutions to extend the lifetime of wireless sensor networks [9]. Power-efficient MAC protocol design is a solution to increase the power efficiency of the network. The trade-off in designing a MAC protocol is between power efficiency and latency. Another solution is low-power circuit design. This kind of lifetime extension solution has limited improvement due to expected functionality.

In this thesis, we investigate a third solution which is using a wake up radio. Monitoring the idle channel wastes a lot of energy in most of the applications of sensor networks [10]. Nodes in monitoring state consume approximately the same amount of power as they use when receiving packets. So, minimizing the monitoring time of the main radio by using a low-power wake up radio will increase power efficiency of wireless networks.

The wake up radio monitors the environment all the time and wakes up the main radio in case of an event. The main radio, which consumes more power for it's operation, is in sleep mode by default. It is just woken up by the wake up radio when there is data to transmit or receive. It sends or receives data and goes back to sleep. Using this scheme, the time that the main radio is in monitoring state is minimized.

Since the wake up radio monitors the channel continuously, the latency is low. The improvement in energy efficiency is because of the fact that the main radio is woken up only when necessary. Typical applications of the dual-radio system are low traffic activity, emergency/on-demand communication, and ultra low power consumption applications. The use of dual-radio architecture results in low access latency, relaxed power budget for the main radio, and relaxed requirements for synchronization.

An important point to consider is that waking up and going to sleep (turning on and off the main radio) consumes energy. Hence, whether it is efficient to use this scheme depends on the event rate. When the event rate is too high, it may be not efficient for the main radio to go back to sleep after each transmission or reception. The best idea to optimize the energy efficiency of the wireless node is to adjust it's operation with the event rate. Dual-radio cooperation makes this goal possible.

Although using a specific MAC protocol for the wake up radio decreases it's energy consumption, we consider the simple case in which the wake up radio is always on. 
This results in less delay since a node does not need to wait for the target to wake up.

We try to calculate the event rate threshold below which the use of wake up radio is efficient and evaluate the improvement in energy efficiency based on mathematical analysis and simulations. We use this result to design the dual-radio cooperation by proposing a rate-adaptive MAC protocol. Dual-radio cooperation makes use of the wake up radio for low event rates and works as a single-radio node model for high event rates.

\section{1-5 Novelty and Contribution}

In this thesis we propose a dual-radio node model which tries to maximize the energy efficiency of the wireless node while achieving a satisfying delay in data transmission. Our focus is the energy waste sources of the MAC sublayer.

We design a new protocol for the dual-radio node to achieve a proper balance between energy consumption and delay. We propose analytical models to compute the wireless node energy consumption and test the results by implementing the designed node model in OPNET. We describe the critical event rate concept for the performance of the dual-radio system. Critical event rate is defined as the rate above which single-radio node model consumes less energy than the dual-radio model. This parameter depends on the radio model characteristics, MAC protocol, and packet size. Based on the critical event rate, we propose a new MAC protocol which adjusts with the event rate. The proposed node model, MAC protocol, and methods of computing the critical event rate are the contributions of this thesis.

\section{1-6 Structure of the Report}

The next chapter reviews the literature about the MAC protocols and topics related to the wake up radio. We describe how these protocols intend to minimize the energy consumption while keeping the delay low. We talk about the applications of wireless sensor networks briefly and focus on a few applications in which using the dual-radio node model results in tremendous energy saving. In the last part of the second chapter, we talk about the MAC and physical layer specifications.

Chapter three introduces the analytical model used to compute the energy consumption of the wireless nodes. The results of this analytical model is demonstrated in the last section of the third chapter.

The fourth chapter presents the designed node model and describes the results of simulations while comparing them with analytical results. We explain the results, conclude, and make final discussion to optimize the model. In the last section, the rate-adaptive MAC protocol is explained.

The last chapter summarizes the conclusions and proposes the future work. 


\section{Chapter 2}

\section{Literature Review}

\section{2-1 Overview}

Nodes in a wireless sensor network are battery-powered. In most of the applications the number of nodes in a network is large so that replacing batteries is not a simple task. Therefore, energy efficiency is a critical issue in wireless sensor networks. Besides, sensor network applications usually generate low traffic load so that the communication channel is expected to be idle most of the time [11]. Thus, idle listening is the most significant source of energy dissipation. Medium Access Control (MAC) is the glue to all solutions of the mentioned problem [12]. MAC is part of the link layer in OSI layer model and is central to the proper functioning of any communication system. The main role of the MAC is to coordinate the access to a medium common to several nodes. Proper MAC rules minimze collisions and interference in a wireless sensor network.

In the following section, we talk about some of the MAC protocols proposed to optimize the communication in a wireless medium and categorize them. Then, we briefly discuss some applications of wireless sensor networks, and focus on two of them to show how much improvement results when using wake up radio. In the last section, MAC and physical layer specifications of the node model are described.

\section{2-2 MAC Protocols}

When a number of wireless nodes use the same communication medium, there should be a protocol that they follow to optimize the access to the shared channel. MAC protocols are designed for adjusting the medium access between the nodes. Each MAC protocol intends to find a proper method to increase the energy efficiency and decrease the packet delivery delay. Decreasing the energy consumption is a result of smaller number of collisions, less idle listening time, and less overhearing and overhead. 
In energy-efficient MAC protocols for sensor networks, to reduce idle listening, nodes sleep for long periods of time instead of being active permanently. Other energy waste sources are overhearing, collisions, overhead, and traffic relay. General principles are to introduce sleep and wake times, use of scheduling (TDMA) or contention or a combination of both, and use of in-channel or out-of-band signaling to reduce overhearing. Usually, there is a trade-off between energy efficiency and fairness, throughput or latency. We first talk about traditional MAC families and disscuss the inefficiencies associated with them [12].

\section{2-2-1 Traditional MAC Families}

Reservation-based and contention-based MAC are the two main approaches for regulating access to a shared wireless medium in traditional protocols.

\section{Reservation-based MAC Protocols}

In reservation-based MAC Protocols, channel access is given to a specific node based on a reserved schedule. This approach requires knowledge of the network topology to establish a schedule that regulates the access of nodes to the channel. Time Division Multiple Access (TDMA) is a good example of this category. In TDMA, channelization of users in the same band is achieved through separation in time. Each user is allowed to transmit in predetermined time slots. There is no need for control packets after group arrangement. There is no interference between adjacent wireless links. So, the energy waste coming from packet collisions is eliminated.

The synchronization requirement and low bandwidth utilization are the problems of TDMA. Inter-group communication is not good, and group membership changes are handled badly. Both knowledge of topology and strict synchronization require large overheads and expensive hardware.

\section{Contention-based MAC Protocols}

In contention-based MAC protocols, nodes contend for medium access. These protocols are mainly based on Carrier Sense Multiple Access (CSMA) [13]. CSMA is a MAC protocol which verifies the absence of any traffic before transmitting on a shared channel. When a node has data packets to send, it uses feedback from a receiver that detects a carrier wave before sending. If the node senses a carrier, it waits until the end of the ongoing transmission before initiating it's own transmission.

ALOHA is a reperesentive scheme of this group of protocols. When nodes want to transmit data they should compete for wireless channel. Colliding nodes will back off for a random time before attempting to access the channel for the next time. In contention-based MAC, data length is signaled to the nodes so that they know how long to defer. CSMA does not rely on a central node and is robust to node mobility. 


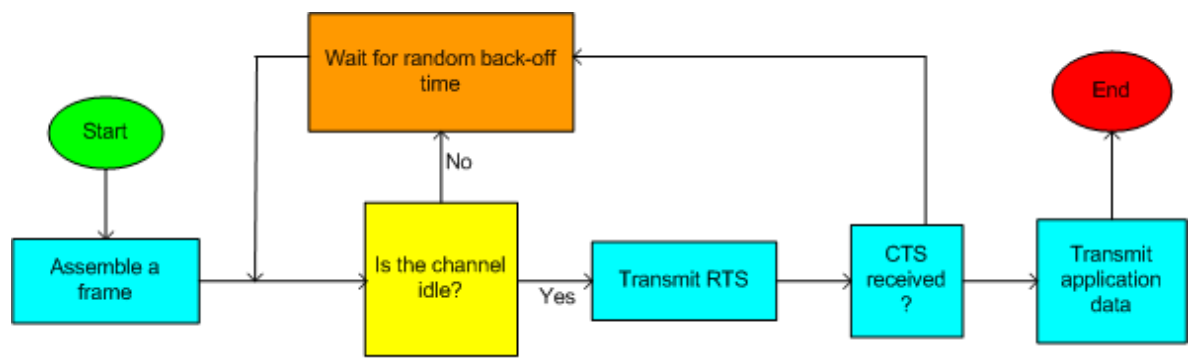

Figure 2-1: CSMA/CA algorithm: A node intending to send data, checks whether the channel is free or not. If the channel is free, the node sends Request To Send, and transmission of the data packet is done after the reception of Clear To Send.

High cost of control overhead and high power consumption in idle mode are the disadvantages of this group. Degraded performance in terms of throughput when the traffic load increases is another problem of these protocols [14].

\section{MAC Protocols for Wireless Sensor Networks}

In order to propose a MAC protocol proper for a wireless sensor network, we should first detect the main causes of energy waste. As discussed briefly in the introduction, Collisions, overhearing, overhead, and idle listening are the most important causes of energy consumption that are related to data link layer. Collisions occur when two or more nodes, which are in the transmission range of each other, transmit simultaneously. Overhearing happens when a node drains energy receiving irrelevant packets. Protocol overhead results in energy waste when transmitting and receiving control packets. For instance, RTS (Request To Send) and CTS (Clear To Send) control packets used in some protocols do not carry useful information and are used to reserve the medium. Idle listening is the situation in which the node does not know when it is the receiver of a packet. Hence, it consumes a lot of energy to monitor the idle channel.

Based on this information, some methods have been proposed to decrease the energy consumption of nodes in a wireless sensor network. These protocols focus on the causes of energy waste, and try to minimze them.

In order to reduce collisions, Carrier Sense Multiple Access with Collision Avoidance (CSMA/CA) is proposed which makes use of RTS/CTS messages. In CSMA/CA a node intending to send data, listens to the channel to see if it is free. If the channel is sensed to be idle, the node sends RTS for the destination. Data packet is sent after CTS reception. Figure 2-1 shows the CSMA/CA algorithm by using a simple block diagram.

The probability of collision of RTS and CTS packets is low since they are small in size [15]. The role of the CTS is to reserve the channel around the receiver node. Although the RTS/CTS procedure decreases collisions in wireless networks, it has some drawbacks. First, data packet sizes are usually small in sensor networks so that their collision probability is in the same order as for RTS/CTS packets. Second, 
it increases energy consumption of the protocol. Third, RTS/CTS packets can only be used in unicast transmission.

Multiple Access Collision Avoidance (MACA) improves CSMA/CA by adding a random back-off time before transmitting RTS in order to avoid collisions which are results of forwarding data simultaneously by multiple neighbors [16]. Like CS$\mathrm{MA} / \mathrm{CA}$, it is proposed to reduce collisions.

CSMA/ARC (Adaptive Rate Control) is a MAC protocol designed to reduce overhead [17]. In contrast to CSMA/CA, CSMA/ARC does not make use of RTS/CTS control messages. It applies a back-off which is shifted according to application periodicity. It also avoids explicit ACK, and considers the data packet to be successfully received when the receiver forwards it to the next hop.

Power-Aware Multi-Access with Signaling (PAMAS) is a MAC protocol proposed to decrease overhearing [18]. It is based on MACA, but uses a separate channel for RTS/CTS exchange and informs about the duration of the transmission. Therefore, a node which is not involved in the ongoing transmission switches its radio off and avoids overhearing. PAMAS is designed for adhoc networks with high traffic load in which tranmissions are frequent. However, the situation is different in sensor networks due to their fairly low traffic load.

Reducing idle listening time is the main target of some protocols. IEEE 802.11 Power Save Mode (PSM) targets energy saving in Wireless Local Area Networks (WLAN) [12]. If there is an access point, a node spens most of the time sleeping, and wakes up periodically to receive beacons from the access point. When there is data destined to a specific node, the access point buffers the data if the node is in the sleep mode. If there are no access points, nodes transmit periodic beacons to keep synchronization in a distributed way.

From the above discussion about the MAC protocols, it can be inferred that wireless sensor networks need specific protocols based on their energy waste sources and applications. In the next section, we discuss some MAC protocols which propose energy efficient solutions for wireless sensor networks. First, we explain scheduled protocols. Then, protocols with common active periods are discussed. Finally, preamble sampling protocols are introduced. These protocols are proper for periodic high-load traffic, medium-load traffic, and rare reporting events respectively. Hybrid protocols are introduced in the last part of this section.

\section{2-2-2 Scheduled Protocols}

Scheduled protocols are based on reservation-based protocols and are variants of TDMA combined with Frequency Division Multiple Access (FDMA). In TDMA, when the schedule is set up, there will be no collisions, no overhearing, and minimized idle listening. However, there are some shortages with scheduled protocols when used in wireless sensor networks. Scalability of collision-free slot assignment is a serious problem. Broadcast communication is another problem which is solved if the protocol is sender-scheduled. Reduced flexibility and large requirement of memory (since knowledge of two-hop neighborhood topology is needed) are further 
difficulties in scheduled protocols. The lack of a central access point results in complexity and high cost for maintaining synchronization and distributing slots.

In TDMA, the scheduler can schedule communication links, senders, or receivers. Time Synchronized Mesh Protocol (TSMP) is a TDMA-based protocol which employs FDMA and frequency hopping in addition, in order to achieve robustness against interference [19]. Access is controlled by frame, which is a tunabel amount of time slots, and nodes can participate in multiple frames.

Some scheduled MAC protocols use centralized scheduling at the sink. In Power Efficient and Delay Aware Medium Access Control protocol for Sensor networks (PEDAMACS), the sink gathers information about traffic and topology during the setup phase [20]. Then, it calculates a global scheduling and sends it to the entire network. BitMAC and Gateway MAC (G-MAC) are other examples of this type $[21],[22]$.

An example of distributed scheduling is Self-organizing Medium Access Control for Sensor networks (SMACS) which allows nodes to establish a communication infrastructure between neighboring nodes by defining transmission and reception slots [23]. Thus, it does not need a master node, and is considered a localized and distributed protocol. Neighbor discovery and channel assignment are the two phases of SMACS. When a link is formed between two nodes, they establish transmission and reception slots.

Traffic Adaptive Medium Access protocol (TRAMA) determines a collision-free scheduling and performs link assignment based on the expected traffic [24]. It consists of two phases: localized topology formation and scheduled channel access. Idle listening and overhearing is decreased by the scheduled channel access which allows each node to be active only when it has to send or receive data. Flow-Aware Meduim Access protocol (FLAMA) and micro MAC use similar ideas [25]. Energy Efficient MAC (EMACs) defines three types of nodes which have different tasks, and divides each slot into three parts [26]. It makes use of distributed scheduling for slot assignment. Position-enabled MAC (PMAC) is similar to EMACs, but adds a new priod in the slot structure for position estimate request from dynamic nodes [27].

In some protocols, the role of nodes change during time. Power-Aware Clustered TDMA (PACT) and Bit-Map Assisted (BMA) are protocols in which nodes alternate in acting as access point, controlling TDMA slots of their neighbors [28], [29]. However, complexity of the algorithm and the overhead of the control phase are the drawbacks of this group.

Mobility adaptive MAC (MMAC) builds a collision-free schedule based on estimation of traffic flow, mobility and dynamicity patterns [30]. This protocol is designed to handle node mobility. The main disadvantage is the need for the knowledge of the position, which results in large energy consumption. Flexible MAC (FlexiMAC) is also able to cope with some node mobility [31]. A contention period is defined to build a data gathering tree rooted at the sink. Slot assignment is done according to the tree. Each node uses three lists for slot assignment and maintenance: Receive Slot List, Transmit Slot List, and Conflict Slot List. Although parent-child 
and child-parent communication is optimal, the tree structure lacks robustness and optimality.

There are protocols adapting to traffic changes. Pattern MAC (PMAC) adapts to traffic changes by going to sleep mode when there is no traffic, but it does not decrease idle listening during active periods. In PMAC schedules are determined based on a node's own traffic and it's neighbors' traffic load [32]. Hence, PMAC increases energy saving by adaptively determining wake up-sleep times.

Off MAC (O-MAC) is a TDMA protocol in which each node independently selects its reception slot [33]. The key idea is to find non-overlapping slots to avoid waking up other nodes uselessly.

Lastly, YMAC is a multi-channel MAC protocol which achieves effective transmission of bursty messages under high traffic conditions, while maintaining low energy consumption [34]. It is primarily designed to decrease latency.

\section{2-2-3 Protocols With Common Active Periods}

In this type of protocols, nodes with common active and sleep priods are used. Therefore, a certain level of synchronization is necessary. During the active time, nodes contend for the channel using contention-based techniques. These protocols are not suitable for irregular traffic applications since the probability of collision is high when contending for the channel in heavy traffic load.

Sensor MAC (SMAC) is the important example of this kind. SMAC is specially designed for wireless sensor networks [35]. It reduces energy waste from all major sources: idle listening, collision, overhearing, and control overhead. It consists of periodic listen and sleep (TDMA), collision and overhearing avoidance (contention), and message passing. At the start time, each node listens for a schedule. If it finds one, it will join the schedule. If after a specific duration it does not find any schedules, it sets a schedule for itself and announces it. If after inventing one's schedule a new one is received, the node takes the new schedule in case no neighbors were found before. Otherwise, if both schedules are useful, it may follow both. When a node has data to send, it waits until the listen time of the target. Then access is achieved by using RTS/CTS messages. During the tranmission, they do not follow their sleep schedule.

SMAC is more efficient than CSMA/CA with reservation since each message is fragmented into many packets and RTS/CTS are used only for the first packet. Each received data packet is acknowledged and a duration field is put in each RTS/CTS/ACK. SMAC is relatively complex and has increased latency. However, good energy performance at light load and adaptive listening which adjusts to traffic conditions, and the ability to achieve the same performance as no-sleep in heavy load, are it's advantages.

In order to increase flexibility and solve the problem of idle listening, the Timeout MAC (TMAC) protocol is designed [36]. TMAC is an improvement to SMAC to reduce energy consumption in idle listening. TMAC dynamically adapts a listen/sleep 
duty cycle through fine-grained timeouts with minimum complexity. All messages are transmitted in variable length bursts and the length of bursts is dynamically determined.

UMAC is another protocol which is based on SMAC, but provides three improvements on this protocol [37]. It provides various duty cycles, utilization-based tuning of duty cycle, and selective sleeping after transmission. In fact, each node uses a utilization function to tune its duty cycle. UMAC improves energy efficiency as well as end-to-end delay.

\section{2-2-4 Preamble Sampling Protocols}

In preamble sampling protocols each node spends most of the time in the sleep mode and wakes up only for a short period to check if there is a transmission on channel. Each data frame is preceded by a preamble that is long enough so that all receivers detect it. STEM-B and STEM-T, which were introduces in the first chapter, are two examples of this type of protocols.

\section{2-2-5 Hybrid Protocols}

Hybrid MAC protocols divide the access channel into two parts. Control packets are sent in the random access channel, and data packets are transmitted by the scheduled channel. Indeed, hybrid protocols combine the previous categories in order to take advantage of their characteristics. Contention-based protocols are more useful for small netwokrs while scheduled protocols are better choices for large networks.

In ZMAC, which is an integration of TDMA and CSMA, a node has a guaranteed access to it's own slot, and contention-based access to other slots [38]. So, collisions are reduced and better energy performance is achieved. ZMAC defines a hybrid method that works based on CSMA in low traffic and switches to TDMA in high traffic conditions.

AMAC is a hybrid of CSMA and TDMA protocols, and is designed mainly for long surveillance and monitoring applications. The main feature of AMAC is that nodes are notified in advance when they will be receivers of packets [39].

As discussed above, numerous MAC protocols have been designed and proposed to minimize the energy consumption and delay. Each of them may perform better than the others in a specific application. Although wake up radio does not need a sophisticated MAC protocol, this information is necessary to clarify the medium access issue and the effect of data link layer functionality on energy efficiency and delay.

The next section talks about MAC protocols for dual-radio node model. 


\section{2-2-6 Wake-up Radio MAC}

There are not so many MAC protocols proposed for the wake up radio. The wake up radio's power consumption is small and it does not need a comlex MAC protocol. However, using the best MAC protocol results in maximum energy efficiency.

In [40], E2RMAC is suggested for the operation of the dual-radio node. In this protocol, the main radios of all nodes are initially in sleep mode. When a node wants to transmit data, it sends a wake up tone on the wake up radio after a random back-off period. Neighboring nodes receive this tone on their wake up radio, and wake their main radio up. The sender also wakes up its main radio and transmits a packets containing the Receiver-ID which identifies the intended receiver of data. The intended receiver keeps its main radio on and all other nodes go to sleep again. After data transmission, sender turns its main radio to sleep mode. If the receiver takes the packet successfully, it sends an ACK and switches back to sleep mode. Otherwise, it discards the packet and goes to sleep.

E2RMAC has some optimizations: an intermediate node which receives the packet, immediately sends the wake up tone for the next hop and skips the back-off time. Receiving this wake up tone by the previous node can be considered as an implicit ACK. If the previous sender does not receive the wake up tone, it waits for a period and repeats the procedure from the beginning. To avoid extra RTS/CTS messages, which results in large overhead, a sender node receiving ACK does not transmit until the transmission back off which is equal to twice the communication delay. Once receiving an implicit ACK, a sender node neglects wake up tones for one Receiver Timer which is equal to one communication duration. This is due to the involvement of the next hop node in transmission that makes it impossible for the current node to communicate without collision. Another optimization is that the neighboring nodes who wake up by the wake up tone, but go to sleep when receiving ReceiverID, refrain from transmitting and receiving for two communication durations.

There are some drawbacks in E2RMAC. Waking up all neighbors of the sender is one major problem of E2RMAC. In fact, this protocol is proper for the wake up radios which are not capable of mentioning the receiver address in their wake up tones. In other words, E2RMAC is considered a range-based dual-radio MAC protocol in which all the nodes within the communication range of the wake up radio, switch to active state once receiving the wake up tone. Using a low-power identity-based wake up radio seems a better idea in terms of energy efficiency. Furthermore, some of the optimizations proposed are based on assumptions which are not always true. For example, the previous sender may want to transmit data to other neighbors which does not collide with ongoing data transmission of the new sender.

We try to design a protocol based on the assumption that the wake up radio mentions the destination address in the wake up message. So, only the target node turns it's main radio on, when receiving a wake up message.

We have talked about the medium access issue, and described the available methods protocols for single-radio and dual-radio node models briefly. We will use this information in the next chapters to design our node model and MAC protocol. 
The next section mentions some applications of wireless sensor networks to illustrate how important they are in the world of today. In most of the applications of wireless sensor networks, using a low-power wake up radio results in less energy consumption. The improvement is much more in applications with low traffic load where the communication channel is free most of the time.

\section{2-3 Applications of Wireless Sensor Networks}

Wireless sensor networks' application areas can be classified into two groups: eventdriven applications and periodic or query-based applications [1].

In event-driven applications, sensor nodes take the necessary actions only when a specific event happens. Forest fire detection system, target tracking or perimeter protection in border monitoring, surveillance of museums, security of offices and homes and shops, and gas detection systems are examples of the event-driven applications of sensor networks. Intelligent streetlight system is a novel application of event-driven sensor networks. By the use of wake up radio, only the lights which are close to the moving cars turn on. In other times, when no cars are moving in the vicinity of the lights, they will be off so that large power saving is achieved.

Periodic applications are used to measure a quantity based on a predetermined schedule. Monitoring temperature or humidity levels of a field and depletion of minerals in soil are typical applications of periodic or query-based sensor networks. In this category, the benefit of using wake up radio is in the fact that there will be no need for synchronization between nodes. To sum up, some major application areas of wireless sensor networks are described below:

- Industrial monitoring: Landfill ground well level monitoring which makes use of sensor networks to monitor the water levels within all ground wells, flare stack monitoring to accurately monitor methane gas production, and water tower level monitoring are examples of this type.

- Transport and logistics: Sensor technology contributes to better tracking of goods and vehicles. An intelligent transportation system uses advanced and emerging technologies to save lives, time, money, energy, and environment [41]. This intelligent system can be categorized to intelligent infrastructure and intelligent vehicles. In the field of intelligent infrastructure, sensors are used to monitor the road traffic. They also monitor the state of physical infrastructures such as bridges. Sensor networks in intelligent vehicles monitor the weather conditions, analyze the traffic, and enable vehicle-to-vehicle communication.

- Agricultural monitoring: Landslide detection to recognize slight movements of the soil, greenhouse monitoring, and measuring the temperature of windrows for efficient composting are typical applications of sensor networks in agriculture.

- Military applications: Monitoring friendly forces and equipment and ammunition, battlefield surveillance, targeting, battle damage assessment, recognition 
of opposing forces, and biological and chemical attack detection are applications of sensors in military affairs [42].

- Health applications: Telemonitoring of human physiciological data and tracking and monitoring doctors and patients inside a hospital are two important uses of wireless sensor networks in health section [43].

- Smart buildings: Heating, ventilation, and air conditioning systems, lightening, shading, air quality and window control, systems switching off devices, metering, standard household applications like television and washing machines, and security and safety (access control) are major applications of sensor networks in this part.

Environmental monitoring, entertainment, security and surveillance, and smart grids and energy control systems are other applications.

In event-driven applications of wireless sensor networks the improvement of energy efficiency when using wake up radio depends on the event rate and the application itself. If events happen frequently, some or all of the sensors should wake up and go to sleep a lot of times. Hence, in these scenarios using a periodic sleep scheduling may be a better choice.

In periodic or query-based applications, wake up radio may be used to eliminate the need for time synchronization.

In the next section, we are going to focus on a few applications of the dual-radio system and make a simple calculation to show how much energy saving results when using a wake up radio compared to the single-radio model.

\section{2-4 Applications Description}

In this section, we consider two applications of wireless sensor networks in detail. We show how much energy is saved by using the dual-radio node with some simple calculations.

\section{2-4-1 Intelligent Street Lightening}

Intelligent Street Lightening is one of the new applications of wireless sensor networks. In cities with small population and in the hours of night when there are not so many cars in the streets, it is energy wasting to keep the street lights on. Using intelligent street lights which are able to recognize the cars movements, and can communicate with each other to inform the other lights in the street to turn on in the car's path, results in much less energy consumption.

There are 4.4 million street lights in the US ten largest metropolitan statistical areas, which use 3 billion $k W h$ of electricity annually, and produce 2.3 million metric tons of CO2 [44]. Reduction of high costs and greenhouse gas emission are the two main 
motivations to put some effort in innovating new power management methods for street lightening.

The idea is to use intelligent lights which can communicate with each other and wake up other lights in case they sense a car passing the street. In other situations, the lights go to power save mode and decrease their energy consumption. So, all nodes need a low-power wake up radio to send wake up tones when an event occurs. If we want to design an intelligent street lightening system, which is capable of transmitting some data such as the amount of traffic and estimating how much nodes should be in the high power mode, another main radio is needed for each node which should be woken up by the wake up radio when needed.

We can do an approximate calculation based on some simplifying assumptions to estimate how much energy this method saves. Suppose that in a length of $100 \mathrm{~km}$ of the streets of a city, intelligent street lights are inserted with a distance of 10 meters from each other. This means that the city has 10000 lights. Assume that 250 watt lamps are used and each of them work 10 hours a day with full power. This results in $365 \times 10 \times 250 \times 10000=9125 M W h$ energy consumption in a year. Next, we assume that each light is equipped with a sensor node which contains one wake up radio monitoring the street. The lightening system works as the previous system 5 hours a day during crowded hours, and stays in power save mode in the other 5 hours. During this mode, lights use half of their power. When a car passes the wake up radio sends a wake up tone to the neighboring nodes and makes them aware that they should switch to the full-power mode. If the arrival of the cars is a Poisson process with mean one per minute, and the average power consumption of wake up radio is $50 \mu W$ [45], then the total energy consumption of one year is calculated as $6844 M W h$ approximately. This is approximately 25 per cent energy saving.

If we assume that the lights are using a dual-radio model with one main radio beside the wake up radio, and the main radio exchanges more information than just waking up the other lights, the amount of energy saving will be approximately the same.

Improved reliability, efficiency, and performance will result in higher community satisfaction. Although substituting the current lightening system with a new intelligent one that needs complex nodes require investment at first, it has a fast payback because of tremendous amount of energy saving. Using intelligent street lights saves a lot of energy especially during the night and in cities with low population.

\section{2-4-2 Forest Fire Detection}

Forest fires, also known as wild fires, are uncontrolled fires occurring in wild areas and cause significant damage to natural and human resources [46]. The cost of damage for one forest fire may be millions of dollars. So, a proper way to confront this problem is necessary.

Using a wireless sensor network to detect the fire soon and transmit the data of the event to a base station is a method to cease fire expansion. Sensor nodes can collect measurements like relative humidity, temperature, smoke and wind speed to 
determine the forest fire danger rate [47]. The sensor network can be organized to clusters so that each node has a cluster head. Each node is aware of it's location by the use of equipment like GPS. The measurements are sent to the cluster head. The cluster head computes the weather index and transmits it to the manager node via sink. Three types of messages can be generated by the nodes: query response, regular report, and emergence report. Therefore, both periodic data collection and emergency event detection are possible which are dependent on the protocols used by nodes.

In this application, implementing a wake up radio in addition to the main radio for each node is advantageous. Since the rate of the events is low, it is not power efficient that the main radio be in monitoring state for long durations. Hence, a low-power wake up radio is used to sense the environment. Even this wake up radio can follow a sleep schedule and it is not necessary for it to monitor permanently. As mentioned before, the use of wake up radio is especially beneficial in applications with low event rate like forest fire detection. If the event rate is high, it depends on the radio type and delay constraints whether it is better to use the wake up radio or single-radio model.

In [48] three MAC protocols for forest fire detection sensor networks are proposed. In contrast to the sensor network of intelligent street lightening system which has a simple topology (since all nodes are in one line and have equal distance from each other), the network used for forest fire detection has a more complex topology. This makes calculations of power consumption of a typical network more difficult. The method proposed in [48] gives us a value for the energy consumption of such a network. It assumed that $N=100$ sensor nodes are uniformly and randomly distributed over a circular area. All nodes transmit their data to the cluster head in case an event occurs. The current dissipation values during transmission, reception, and sleep modes are $46 \mathrm{~mA}, 16 \mathrm{~mA}$, and $6.5 \mu \mathrm{A}$ respectively. The data rate is 1.2 kbps and each message is 8 bytes long. Each node generates information about it's measurements every 30 minutes.

The duration of a message, $T_{m s g}$, is computed as below:

$$
T_{m s g}=\frac{8}{1.2}(\text { bytes } / \text { kbps })=53.33 m s
$$

We can compute the energy required for sending a message, $E_{s}$, and energy consumed in receiving a message, $E_{r}$ :

$$
\begin{aligned}
& E_{s}=T_{m s g} \times 46 m A \times 3 v \\
& E_{r}=T_{m s g} \times 16 m A \times 3 v
\end{aligned}
$$

For the simple circular topology, it is possible to determine the number of nodes that are 1 hop away from the sink and the same for more hops. Assume that $N_{1}, N_{2}, N_{3}$, and $N_{4}$ are the number of nodes which are 1, 2, 3, and 4 hops away from the sink respectively. If we define:

$$
N_{t r}=4 \times N_{4}+3 \times N_{3}+2 \times N_{2}+1 \times N_{1}
$$


Then, we can compute a typical value for the total consumed energy, $E$, during 30 minutes assuming that each node generates information every 30 minutes:

$$
E=\left(E_{s}+E_{r}\right) \times N_{t r}+\left((60 \times 30) \times N-2 \times N_{t r} \times T_{m s g}\right) \times 16 m A \times 3 v
$$

Collisions are not taken into account in this model. We have assumed that the nodes are always in the active mode and there is no duty cycle. If we assume that $N_{1}, N_{2}, N_{3}$, and $N_{4}$ are $5,15,30,50$ repectively, we see that the average power consumption of a node, $P$, is:

$$
P=\frac{E}{N \times 30 \times 60}=48 \mathrm{~mW}
$$

Considering the implementation costs beside these values, yet it seems efficient to set up such networks to prevent forest fires which cause a lot of damages.

If we use the same model for a sensor network with wake up radio with average current consumption of $9 \mu \mathrm{A}$, the values for total energy and average power consumption will be calculated as:

$$
\begin{gathered}
E_{\text {wakeup }}=\left(E_{s}+E_{r}+E_{\text {turn-on }}+E_{\text {turn-off }}\right) \times N_{\text {tr }}+((60 \times 30) \times N \\
\left.-2 \times N_{\text {tr }} \times T_{m s g}\right) \times(6.5 \mu A+9 \mu A) \times 3 v \\
P_{\text {wakeup }}=\frac{E_{\text {wakeup }}}{N \times 30 \times 60}=65 \mu \mathrm{W}
\end{gathered}
$$

In which $E_{\text {turn-on }}$ and $E_{\text {turn-off }}$ are the energy values required for switching the main radio on and off, and are assumed to be $0.5 \mathrm{~mJ}$. This value is high for switching energy, but here we have considered the worst case. We have assumed that nodes only turn their main radio to active mode when there is data to send or receive, and all other time the main radio is inactive and the wake up radio listens to the channel. Again we will see huge energy saving which is more than 99 per cent.

In the next section, we talk about the Characteristics of the wake up radio.

\section{2-5 MAC and Physical Layer Specifications}

Typical application examples of dual-radio system are emergent/on-demand communication, low traffic activity, and ultra low power consumption. Due to the requirements of these applications, a practical wake up radio should satisfy some conditions [49]: it should have standard components so that the development becomes faster. It should have low cost, low power consumption, and low delay to help the sensor network achieve it's cost, power, and delay specifications. It should also have low interference and ten meters range. Based on these requirements, a practical wake up radio is proposed in [49].

The wake up circuitry uses On-Off Keying (OOK) with a symbol rate of $862 \mathrm{~Hz}$. OOK is a digital modulation technique and is the simplest form of Amplitude-Shift 
Keying (ASK) modulation that represents digital data as the presence or absence of a carrier wave.

The wake up radio operates in the European license-free $868 \mathrm{MHz}$ band. The main source of interference is GSM equipment transmitting in $900 \mathrm{M} \mathrm{Hz}$ band. GSM also uses OOK, but at a lower frequency of $200 \mathrm{~Hz}$. The wake up radio can switch at a maximum frequency of $900 \mathrm{~Hz}$ which makes the discrimination possible. The total price and power consumption of the proposed wake up radio amount to 5.39 Euro and $171 \mu W$ respectively.

In [9], IMEC's dual-radio proposal is described. The main radio and the wake up radio both work in the ISM band, 2.4-2.485 $\mathrm{GHz}$, with possible 2.36-2.4 $\mathrm{GHz}$ MBAN extension. The hardware of the two radios can be shared. Scalable data rate is ranged from $16 \mathrm{kbps}$ to $16 \mathrm{Mbps}$. Receiver sensitivity should be better than -75 dBm. OOK/GFSK transmission supports Direct Sequence Spreading Spectrum (DSSS). Root raised cosine or Gaussian is used as the reference pulse shaper. Optional error correction is used to achieve flexibility and reliability. Cyclic Redundancy Check is used to verify the packet integrity.

IMEC's narrowband MAC proposal includes two parts. A beacon-enabled mode which is a priority-guaranteed MAC protocol, and a non-beacon/emergency mode as the wake up radio enhancement. High quality of service (QoS), high scalability, support of different topologies, and broadcast and multicast capabilities are the advantages of this model. The targeted applications for the proposed dual-radio system are medical applications and $\mathrm{CE}$ applications. Combination of wake up receiver and priority-guaranteed MAC protocol provides high energy efficiency and prompt downlink and uplink access for medical applications. Application-specific control channels in priority-guaranteed MAC enable QoS differentiation. Collision-free data channel improves energy efficiency for high speed CE applications. Furthermore, an adaptive frame structure is proposed which provides high flexibility and scalability. Dedicated control channels facilitate complex signaling exchange for multi-hop extension. The ultra low power wake up receiver is a bit-rate scalable (10 kbps- 1 Mbps) OOK wake up receiver which is used to identify wake up calls. It aims at two QoS requirements: low access latency and low energy consumption.

For our simulations, we should define the OSI layers specifications. We should select a MAC protocol for the dual-radio node. E2RMAC, which was described in the previous sections, is a MAC protocol for a range-based dual-radio node. The wake up radio is always monitoring the channel. When it receives a wake up tone, it wakes up the main radio for data reception. The source node first sends a packet containing the address of the receiver by it's main radio. All other nodes go to sleep again, and the destination gets data from the sender. It may send an ACK to the sender or send a wake up tone for the next hop transmission which is considered as an implicit ACK for the previous sender.

Another possible MAC which requires identity-based wake up radio is a MAC protocol in which the address of the receiver is included in the wake up signal. In this case, only the destination wakes it's main radio up so that a lot of energy for switching the main radio of the neighbors is not wasted. Again we can either use ACK or consider the wake up message of the next hop as an implicit ACK. 
For the main radio model, we consider three radio types with various power consumptions to compare the performance of the dual-radio node with them for different cases. The wake up radio model that we focus on, is the IMEC proposal described above, which consumes $50 \mu W$ power constantly.

The next chapter is allocated to describe the analytical model for calculating the energy consumption of the wireless node. 


\section{Analytical Model}

\section{3-1 Overview}

In this chapter, we propose methods to calculate the energy consumption of singleradio and dual-radio wireless nodes. For the dual-radio model, we describe the mathematical model for both identity-based and range-based wake up receivers. We will compare the results to see the energy efficiency of the dual-radio model.

First, we talk about a method to calculate the energy consumption of a node. Then, the analytical model is demonstrated by plotting the results for a typical wireless node.

\section{3-2 Analytical Model For Node Energy Consumption}

In [50], it is proposed that the total energy consumption of a node can be expressed as:

$$
E(t)=N_{T}(t) E_{T}+N_{R}(t) E_{R}+T_{S}(t) P_{S}+T_{I}(t) P_{I}
$$

In which $N_{T}(t)$ and $N_{R}(t)$ are the number of times that a node transmits and receives a packet during time $\mathrm{t}, T_{S}(t)$ and $T_{I}(t)$ are the total time that a node spends in sleep and idle states within period $t, P_{S}$ and $P_{I}$ are the power consumption during sleep and idle modes. $E_{T}$ and $E_{R}$ are the energy required to transmit and receive one packet.

Using this basic equation, we can compute the energy consumption of a node for various MAC protocols. $N_{T}(t)$ and $N_{R}(t)$ depend on the event rate and the period for which we are computing the energy consumption. $P_{S}$ and $P_{I}$ depend on the radio model that is chosen. $E_{T}, E_{R}, T_{S}(t)$, and $T_{I}(t)$ are the parameters that depend on the MAC protocol. We use this basic formula to compute the energy consumption of a single-radio node which works based on SMAC and Zigbee, and a dual-radio node with range-based and identity-based wake up radio. 


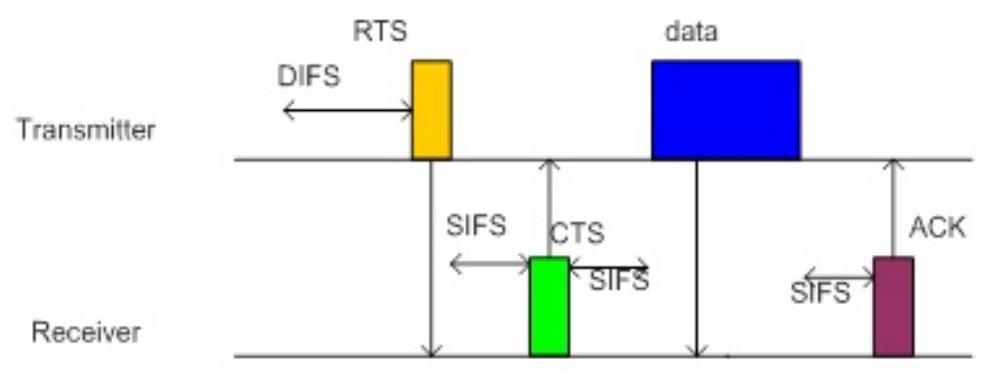

Figure 3-1: SMAC protocol: SMAC consists of periodic listen and sleep, collision and overhearing avoidance, and message passing. Nodes follow a sleep schedule and make use of Request To Send and Clear To Send messages for data exchange.

\section{3-2-1 SMAC Node Energy Consumption}

First, we focus on SMAC protocol. As deiscussed in the second chapter, SMAC is made up of periodic listen and sleep (TDMA), collision and overhearing avoidance (contention), and message passing. After taking or inventing a schedule, the wireless node can take part in data exchange. When a node has data to send, it waits until the active time of the target. Then, access is achieved by using RTS/CTS messages. During the tranmission, participants do not follow their sleep schedule.

Figure 3-1 depicts how SMAC protocol works. Short InterFrame Space (SIFS) is the small time interval between RTS and CTS, data packet and CTS, and data packet and acknowledgements. DCF InterFrame Space (DIFS) is the time that a node, which has data to send, waits before sending RTS.

We calculate the energy consumption of SMAC by computing the parameters used in the mentioned equation for total node energy consumption. $E_{T}$ and $E_{R}$ for SMAC are computed as [50]:

$$
\begin{array}{r}
E_{T}=P_{T x}\left(t_{R T S}+t_{\text {data }}\right)+P_{R x}\left(t_{C S}+t_{B O}+t_{S L}+t_{C T S}+t_{A C K}\right. \\
\left.+3 t_{S I F S}+t_{D I F S}\right) \\
E_{R}=P_{T x}\left(t_{C T S}+t_{A C K}\right)+P_{R x}\left(t_{R T S}+t_{d a t a}+3 t_{S I F S}+t_{D I F S}\right)
\end{array}
$$

$t_{R T S}, t_{d a t a}, t_{C S}, t_{B O}, t_{S L}, t_{C T S}, t_{A C K}, t_{S I F S}, t_{D I F S}$ are the times spent in sending RTS, sending data, carrier sense delay, back-off delay, sleep delay, receiving CTS, receiving ACK, SIFS, and DIFS, respectively. Assuming that each node has Poisson arrival rate of transmitting packets with mean $\lambda_{T}$, and Poisson arrival rate of receiving packets with mean $\lambda_{R}$, the number of packet transmissions and receptions can be computed as:

$$
\begin{aligned}
& N_{T}(t)=\lambda_{T} t \\
& N_{R}(t)=\lambda_{R} t
\end{aligned}
$$

The number of packet receptions depends on the position of a node in the network and is not equal for all of the nodes. For instance, in a network in which all nodes should send their data packets to a central sink, a node close to the sink has 
higher arrival rates, because it should relay messages of other nodes to the sink. Considering this point we can define the packet transmission and reception rate of node $i$ as $\lambda_{T i}$ and $\lambda_{R i}$.

The sleep time and the idle listening time during period $t$ for SMAC are calculated as $[50]$ :

$$
\begin{array}{r}
T_{S}=p_{\text {succ }}\left[\left(\left(\sum_{i=1}^{n} \lambda_{T i}-\lambda_{R}\right) t\right) t_{\text {data }}+\left(\left(\sum_{i=1}^{n} \lambda_{R i}-\sum_{i=1, j=1, i \neq\{j\}}^{n} \lambda_{R i j}\right.\right.\right. \\
\left.\left.\left.-\lambda_{T}\right) t\right) t_{\text {data }}\right]+\left[t-\left(\left(\left(\sum_{i=1}^{n} \lambda_{T i}-\lambda_{R}\right) t\right) t_{\text {data }}+\left(\left(\sum_{i=1}^{n} \lambda_{R i}\right.\right.\right.\right. \\
\left.\left.-\sum_{i=1, j=1, i \neq\{j\}}^{n} \lambda_{R i j}-\lambda_{T}\right) t\right) t_{\text {data }}+N_{T}\left(t_{R T S}+t_{\text {data }}\right. \\
\left.+t_{C S}+t_{B O}+t_{S L}+t_{C T S}+t_{A C K}+3 t_{S I F S}+t_{D I F S}\right) \\
\left.\left.+N_{R}\left(t_{C T S}+t_{A C K}+t_{R T S}+t_{\text {data }}+3 t_{\text {SIFS }}+t_{D I F S}\right)\right)-\varepsilon\right] \frac{T_{\text {sleep }}}{T_{\text {frame }}} \\
T_{I}=\left[t-p_{\text {succ }}\left(\left(\left(\left(\sum_{i=1}^{n} \lambda_{T i}-\lambda_{R}\right) t\right) t_{\text {data }}+\left(\left(\sum_{i=1}^{n} \lambda_{R i}-\sum_{i=1, j=1, i \neq\{j\}}^{n} \lambda_{R i j}\right.\right.\right.\right.\right. \\
\left.\left.\left.-\lambda_{T}\right) t\right) t_{\text {data }}\right)+N_{T}\left(t_{R T S}+t_{\text {data }}+t_{C S}+t_{B O}+t_{S L}+t_{C T S}+t_{A C K}\right. \\
\left.+3 t_{S I F S}+t_{D I F S}\right)+N_{R}\left(t_{C T S}+t_{A C K}+t_{R T S}+t_{\text {data }}+3 t_{S I F S}\right. \\
\left.\left.\left.+t_{D I F S}\right)\right)-\varepsilon\right] \frac{T_{\text {listen }}}{T_{\text {frame }}}+\varepsilon
\end{array}
$$

$n$ is the number of neighboring nodes. The packet transmitting rate of neighbor $i$ is $\lambda_{T i}$ and the packet receiving rate of neighbor $i$ is $\lambda_{R i}$. $\lambda_{R i j}$ is the packet receiving rate of node $j$ from neighbor $i$. $p_{\text {succ }}$ is dependent on the probability that a transmission is successful and the node is listening or not. $\varepsilon$ represents the total extra idle period when a node changes it's mode back to periodic listen and sleep schedule. $\varepsilon$ and $p_{\text {succ }}$ are derived as:

$$
\begin{aligned}
\varepsilon & =N_{I} \frac{T_{\text {frame }}}{2} \\
p_{\text {succ }} & =\frac{n \tau(1-\tau)^{n-1}}{1-(1-\tau)^{n}}
\end{aligned}
$$

$N_{I}$ is the number of times that the extra idle period occurs, and $\tau$ is the transmission probability when $n$ nodes contend on the same channel.

Another important point about the mentioned calculations is that the number of neighbors for all nodes is not equal in many cases. Therefore, for computing the total power consumption of a network, we should use the above equations for each node separately and compute the sum of the results. 


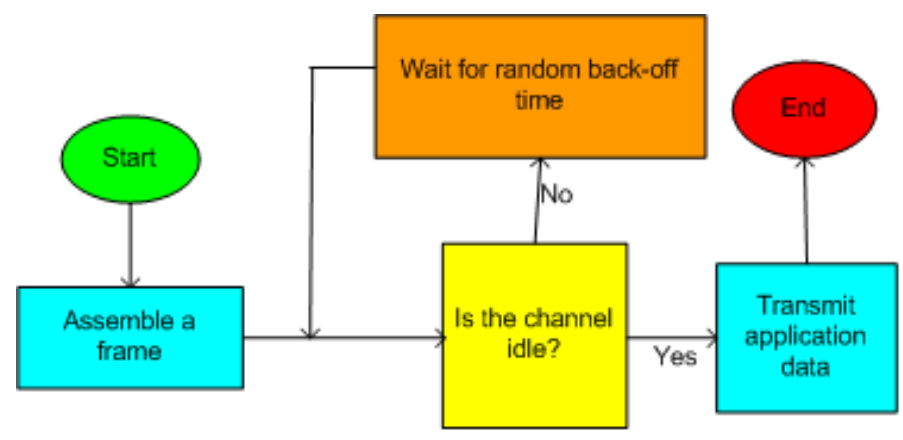

Figure 3-2: Zigbee non-beacon-enabled block diagram: The CSMA/CA process without Request To Send/Clear To Send control messages is used as the MAC protocol of the Zigbee node. A node, intending to send data, listens to the channel, and if it finds the channel free, it sends the data packet. Otherwise, it waits for a random time and starts the process again.

\section{3-2-2 Zigbee Node Energy Consumption}

Zigbee is a specification for a suite of high level communication protocols using small, low-power digital radios based on the IEEE 802.15.4 standard. The current profiles derived from the Zigbee protocols support beacon and non-beacon-enabled networks.

In beacon-enabled networks, the special network nodes called Zigbee routers transmit periodic beacons to confirm their presence to other network nodes. Nodes may sleep between beacons to lower their duty cycle and increase their battery life. In beacon-enabled networks, nodes only need to be active while a beacon is being transmitted.

In non-beacon-enabled networks, an unslotted CSMA/CA channel access mechanism is used. In this type, the power consumption is asymmetrical: some devices are always active while others spend most of their time sleeping.

We use the same idea as SMAC node energy consumption analytical model to propose a model to calculate the energy consumption of a non-beacon-enabled Zigbee node which works based on CSMA/CA and is always active. The energy required to transmit a packet is:

$$
E_{T}=P_{T x}\left(t_{\text {data }}\right)+P_{R x}\left(t_{A C K}+t_{\text {listen }}+t_{p l}\right)
$$

Each node that has data to send, invokes the CSMA/CA process. It senses the channel, and if it is free the node sends data. Otherwise, it waits for a random time and senses the channel again at the end of this time. Figure 3-2 shows the Zigbee non-beacon-enabled node's MAC, by using a simple block diagram.

We define $t_{\text {listen }}$ as the time spent before sending a packet when a node has to report an event. This duration depends on the topology of the network and the number of the neighbors. If we assume that $t_{\text {sense }}$ is the time that each node senses the channel to see if the channel is busy or not, we have:

$$
t_{\text {listen }}=\sum_{i=0}^{Z-1}\left(1-p_{\text {succ }}\right)^{i}\left(p_{\text {succ }}\right)\left(t_{\text {sense }}+t_{\text {backoff }}(i)\right)
$$


Here $p_{\text {succ }}$ is the probability of successful transmission, and $Z$ is the maximum number of back offs that is allowed. $t_{\text {backoff }}(0)$ is zero and for other values of $i$ we can write:

$$
t_{\text {backoff }}(i)=N B(i) \times U N
$$

$N B(i)$ is the number of back-off periods in the ith attempt, and $U N$ is the unit period. The method implemented in OPNET to compute the number of back off periods is that at each time a number is chosen randomly from the values between zero and $2^{x}-1$, in which $x$ is the backoff exponent, and each time the channel is busy this back off exponent is increased by 1 .

The energy consumed in receiving a packet is:

$$
E_{R}=P_{T x}\left(t_{A C K}\right)+P_{R x}\left(t_{d a t a}+t_{p l}\right)
$$

Nodes are always on. So, the sleep time $T_{S}$ is zero. The total time spent in idle state during period $t$ is:

$$
T_{I}=t-\left(N_{T}\left(t_{\text {data }}+t_{A C K}+t_{\text {listen }}+t_{p l}\right)+N_{R}\left(t_{\text {data }}+t_{A C K}+t_{p l}\right)\right)
$$

Inserting these parameters in the basic formula, $E(t)=N_{T}(t) E_{T}+N_{R}(t) E_{R}+$ $T_{S}(t) P_{S}+T_{I}(t) P_{I}$, gives us the Zigbee node energy consumption during time $t$.

\section{3-2-3 Range-based Wake-up Radio}

In this section, we use the same idea to achieve a formula for the power consumption of a range-based wake up radio. Assume that the wake up radio works based on E2RMAC. Figure 3-3 shows how this protocol works. When a node intends to transmit data, it sends a wake up tone on the wake up radio after a random backoff period. All neighboring nodes within the wake up range of the sender, go to active mode. The sender also wakes up its main radio and transmits a packets containing the Receiver-ID which identifies the intended receiver of the data packet by the main radio. The intended receiver keeps it's main radio on for data exchange and all other nodes go to sleep again.

In this case, it is assumed that $P_{I}$ is the power consumption of the wake up radio during idle listening mode, because we have assumed that the main radio only wakes up when it has data to transmit or receive. In other words, the total idle time is the total time that the wake up radio is monitoring the channel. Since the wake up radio is always active, the total sleep time is the total time that the main radio is in sleep mode. We also assume that $p_{\text {succ }}$ is equal to 1 for simplicity.

The required energy for transmission of one packet can be calculated as:

$$
\begin{array}{r}
E_{T}=P_{T x}\left(t_{\text {data }}+t_{\text {address }}\right)+P_{R x}\left(t_{p l}\right)+P_{T x W}\left(t_{\text {tone }}\right)+P_{R x W}\left(t_{B O}\right) \\
+E_{\text {turn-on }}+E_{\text {turn-off }}
\end{array}
$$

$t_{p l}$ is the processing delay. $E_{\text {turn-on }}$ and $E_{\text {turn-off }}$ are the energy required for switching the main radio on and off; $P_{T x W}$ and $P_{R x W}$ are the power consumption of wake 


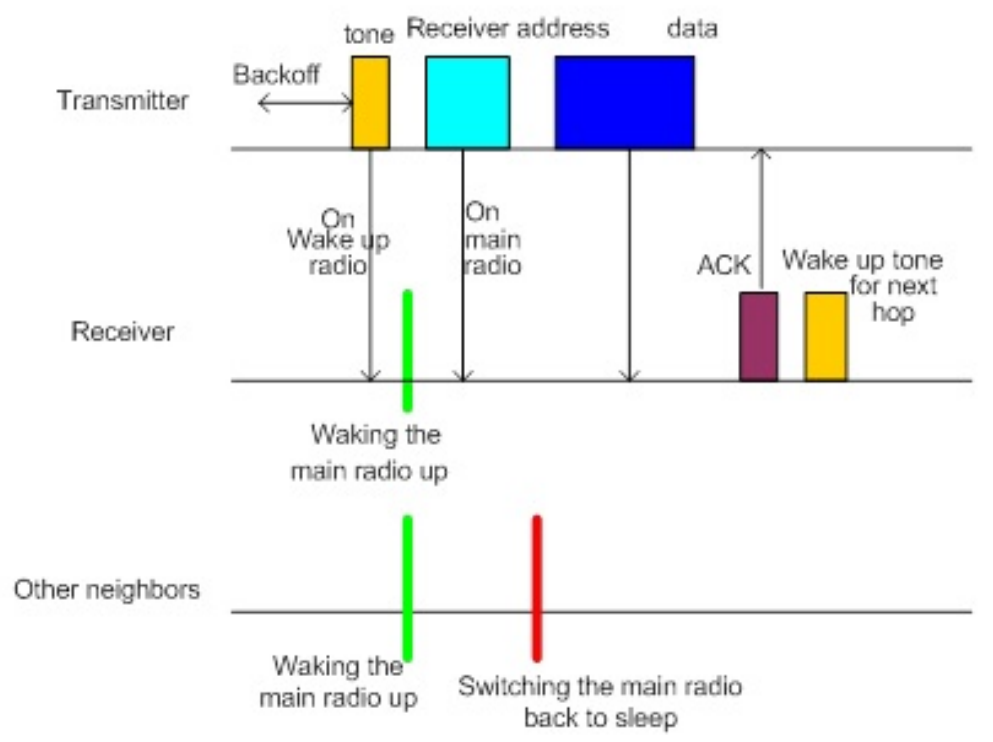

Figure 3-3: E2RMAC protocol: E2RMAC is a MAC protocol for range-based wake up receivers. The wake up tone wakes up all the nodes receiving it, and the first packet sent by the main radio determines the intended receiver.

up radio when transmitting and receiving wake up tones. For the energy consumption of receiving a packet we have:

$$
\begin{array}{r}
E_{R}=P_{R x}\left(t_{p l}+t_{\text {data }}+(L+1) t_{\text {address }}\right)+(L+1) P_{R x W}\left(t_{\text {tone }}\right) \\
+(L+1)\left(E_{\text {turn-on }}+E_{\text {turn-off }}\right)
\end{array}
$$

It is considered that for each message transmission $L$ neighbors of the sender switch their main radio on when they receive the wake up tone, and all of them switch their main radio off when they receive the first message containing the address. We have assumed that the total number of neighbors is $n$, but this parameter depends on the position of the node. So, as before we should compute this for each node in the network separately. The important point to note is that in SMAC when neighbors of a specific node, which are in transmission range of it, receive data packets from other nodes out of range, the node becomes aware. The reason is the transmission of CTS. However, in E2RMAC there is a difference between the number of neighbors of a node whose transmission wakes it up, and the number of neighboring nodes whose reception wakes it up. We assume that the number of neighbors whose reception wakes up the node is $m$ while the number of neighbors whose transmission wakes the node up is $n$. Since the main radio only wakes up when it has to transmit or receive data and when one of the neighbors transmits or receives, we can compute the sleep time during period $t$ as:

$$
\begin{array}{r}
T_{S}=t-\left(\left(\left(\left(\sum_{i=1}^{n} \lambda_{T i}-\lambda_{R}\right) t\right) t_{\text {address }}+\left(\left(\sum_{i=1}^{m} \lambda_{R i}-\sum_{i=1, j=1, i \neq\{j\}}^{m} \lambda_{R i j}\right.\right.\right.\right. \\
\left.\left.\left.-\lambda_{T}\right) t\right) t_{\text {address }}\right)+N_{T}\left(t_{\text {address }}+t_{\text {data }}+t_{B O}+t_{\text {tone }}+t_{\text {pl }}\right) \\
\left.+N_{R}\left(t_{\text {address }}+t_{\text {tone }}+t_{\text {data }}+t_{\text {pl }}\right)\right)
\end{array}
$$


The idle listening time is the time that the wake up radio monitors the channel:

$$
T_{I}=t-\left(N_{R}+N_{T}\right) t_{\text {tone }}
$$

For dual-radio system we consider the wake up radio power consumption as the idle power consumption, and we assume that the wake up radio always consumes the same power no matter it is transmitting, receiving, or monitoring the channel. The sleep power consumption is the power consumed by the main radio during sleep period. By considering these issues we can simplify the above calculations:

$$
\begin{gathered}
E_{T}=P_{T x}\left(t_{\text {data }}+t_{\text {address }}\right)+P_{R x}\left(t_{p l}\right)+E_{\text {turn-on }}+E_{\text {turn-off }} \\
E_{R}=P_{R x}\left(t_{p l}+t_{\text {data }}+(L+1) t_{\text {address }}\right)+(L+1)\left(E_{\text {turn-on }}+E_{\text {turn-off } f}\right) \\
T_{I}=t
\end{gathered}
$$

Combining these results and inserting them into the basic node energy consumption formula, gives the total energy consumption of a range-based wake up radio node model.

\section{3-2-4 Identity-based Wake-up Radio}

In this part, we assume that the address of the destination is in the wake up message. So, all the neighbors of the sender do not switch their main radio on, and the energy waste caused by overhearing and extra switching is eliminated. When a node has data to send, it first sends a wake up tone containing the address of the destination on its wake up radio. Upon receiving this Wake up Request message (which is the same as RTS, but is named Wake up Request to show it's task more clear), the target node sends a CTS message on its wake up radio, and turn its main radio on. After receiving data, the receiver sends an ACK to sender. Figure 3-4 shows the simple MAC protocol used for identity-based wake up radio.

The required energy for transmission and reception of one packet can be calculated as:

$$
\begin{array}{r}
E_{T}=P_{T x}\left(t_{\text {data }}\right)+P_{T x W}\left(t_{\text {tone }}\right)+P_{R x}\left(t_{p l}+t_{\text {ACK }}\right)+P_{R x W}\left(t_{C T S}\right. \\
\left.+t_{\text {listen }}+t_{\text {pl }}\right)+E_{\text {turn-on }}+E_{\text {turn-off }} \\
E_{R}=P_{T x}\left(t_{A C K}\right)+P_{T x W}\left(t_{C T S}\right)+P_{R x}\left(t_{p l}+t_{\text {data }}\right)+P_{R x W}\left(t_{\text {tone }}\right) \\
+E_{\text {turn-on }}+E_{\text {turn-off }}
\end{array}
$$

We have assumed that the node invokes the CSMA/CA process to send RTS. The sleep time and idle listening time are:

$$
T_{S}=t-\left(t_{\text {data }}+t_{A C K}+t_{p l}+t_{\text {listen }}\right)\left(N_{T}+N_{R}\right)
$$




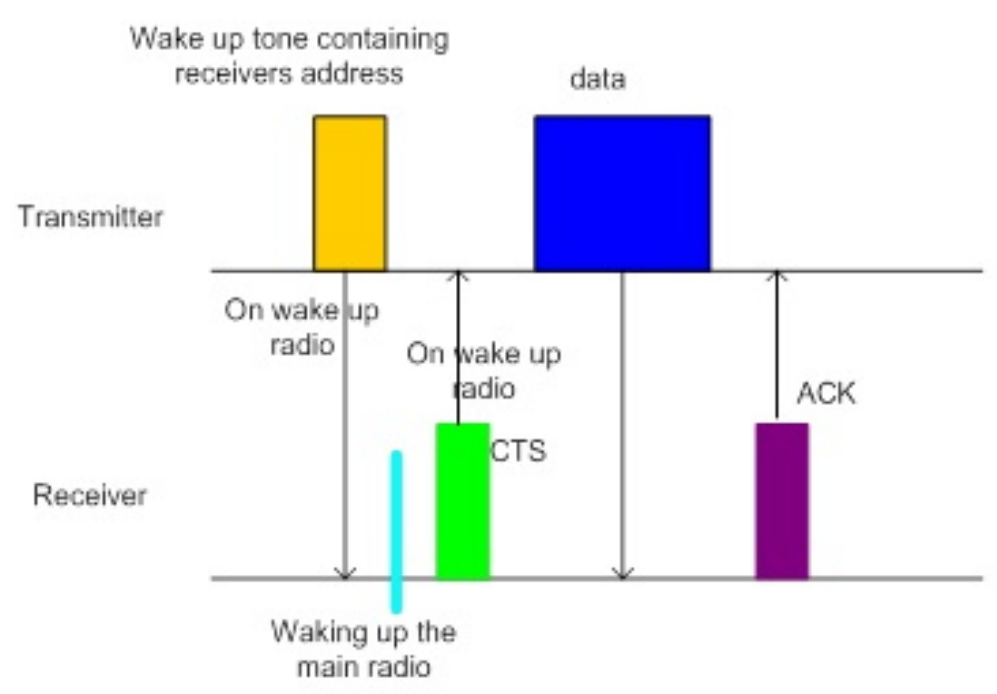

Figure 3-4: Identity-based wake up MAC: Wake up message includes the address of the receiver. So, only the intended receiver turns it's main radio to active mode.

$$
T_{I}=t-\left(N_{R}+N_{T}\right) t_{\text {tone }}
$$

With the same simplifying assumption, that the wake up radio always consumes the same power no matter it is transmitting, receiving, or monitoring the channel, we have:

$$
\begin{gathered}
E_{T}=P_{T x}\left(t_{d a t a}\right)+P_{R x}\left(t_{p l}+t_{A C K}\right)+E_{\text {turn-on }}+E_{\text {turn-off }} \\
E_{R}=P_{T x}\left(t_{A C K}\right)+P_{R x}\left(t_{p l}+t_{\text {data }}\right)+E_{\text {turn-on }}+E_{\text {turn-off }} \\
T_{S}=t-\left(t_{\text {data }}+t_{A C K}+t_{p l}\right)\left(N_{T}+N_{R}\right) \\
T_{I}=t
\end{gathered}
$$

The next section demonstrates the analytical model by plotting it for a typical wireless node.

\section{3-3 Demonstration}

To demonstrate the proposed method, we plot the energy consumption of an end node in a network based on the described analytical model. We plot the node energy consumption for SMAC, Zigbee, and identity-based wake up radio. Since the energy performance of identity-based wake up receivers is much better than the range-based, we choose identity-based wake up radio to investigate the energy efficiency of the dual-radio cooperation. As mentioned before, overhearing and extra switching energy caused by unnecessary wake-ups are the main sources of energy waste in range-based wake up receivers which make us choose identity-based 
receivers. We assume that the wake up radio consumes the constant power of 50 $\mu \mathrm{W}$ [9]. For the main radio we consider three different products. Table 3-1 shows the assumed parameters for the first radio, Chipcon cc1000 [51]. The second and third radios are introduced in the Tables 3-2 and 3-3 [52], [53]. We name these two radios LP (Low Power) radio and IMEC.

Table 3-1: Chipcon cc1000

\begin{tabular}{|l|l|}
\hline transmission power & $36 \mathrm{~mW}$ \\
reception power & $24 \mathrm{~mW}$ \\
idle power & $24 \mathrm{~mW}$ \\
sleep power & $3 \mu \mathrm{W}$ \\
data rate & $75 \mathrm{kbps}$ \\
\hline
\end{tabular}

Table 3-2: LP Radio

\begin{tabular}{|l|l|}
\hline transmission power & $2 \mathrm{~mW}$ \\
reception power & $2 \mathrm{~mW}$ \\
idle power & $2 \mathrm{~mW}$ \\
sleep power & $3 \mu \mathrm{W}$ \\
data rate & $100 \mathrm{kbps}$ \\
\hline
\end{tabular}

The size of the data packet is assumed to be 1024 bits and the size of the control messages are assumed to be 128 bits. The timing values are computed from the size of the packets and the data rate of the radios. The energy required for a transition in states is assumed to be $77.5 \mu \mathrm{J}$ since we have assumed a transition time of $2.5 \mathrm{~ms}$ with power consumption of $31 \mathrm{~mW}$. This value has a prominent effect on the difference in energy consumption between SMAC and protocols which use an additional wake up radio. $P_{\text {succ }}$ is assumed 1 . The packet inter-arrival time has exponential distrobution. This means that the event rate has Poisson distribution. Table 3-4 shows the assumed values for the parameters. Note that we assume that data transmission is the last step of a transmission session, and we do not take acknowledgement into our calculations.

The achieved improvement in using a wake up radio is dependent on the application in which the sensor network is deployed, the typical values of event rate for that specific application, the data and control messages' packet size, the wake up radio model, the power consumption of the main radio during different states and it's data rate, and the switching energy which makes the main radio on and off. In the proposed analytical model, the energy consumption of each node should be computed individually since the data reception and transmission of nodes depend on their position in the network and are not equal to each other.

We estimate the energy consumption of an end device in a simple topology. This node is not the relay for any messages and just reports the event it detects by sending messages to a neighboring node.

In Figure 3-5, the energy consumption of a node which deploys Chipcon cc1000 is depicted for the dual-radio node and a node which uses Zigbee non-beacon-enabled

Table 3-3: IMEC

\begin{tabular}{|l|l|}
\hline transmission power & $200 \mu \mathrm{W}$ \\
reception power & $200 \mu \mathrm{W}$ \\
idle power & $200 \mu \mathrm{W}$ \\
sleep power & $3 \mu \mathrm{W}$ \\
data rate & $200 \mathrm{kbps}$ \\
\hline
\end{tabular}


Table 3-4: Demonstration parameters

\begin{tabular}{|l|l|}
\hline wake up radio power consumption & $50 \mu W$ \\
wake up radio data rate & $1 \mathrm{kbps}$ \\
Wake up Request size & $128 \mathrm{bits}$ \\
CTS size & $128 \mathrm{bits}$ \\
data packet size & $1 \mathrm{kbits}$ \\
\hline
\end{tabular}

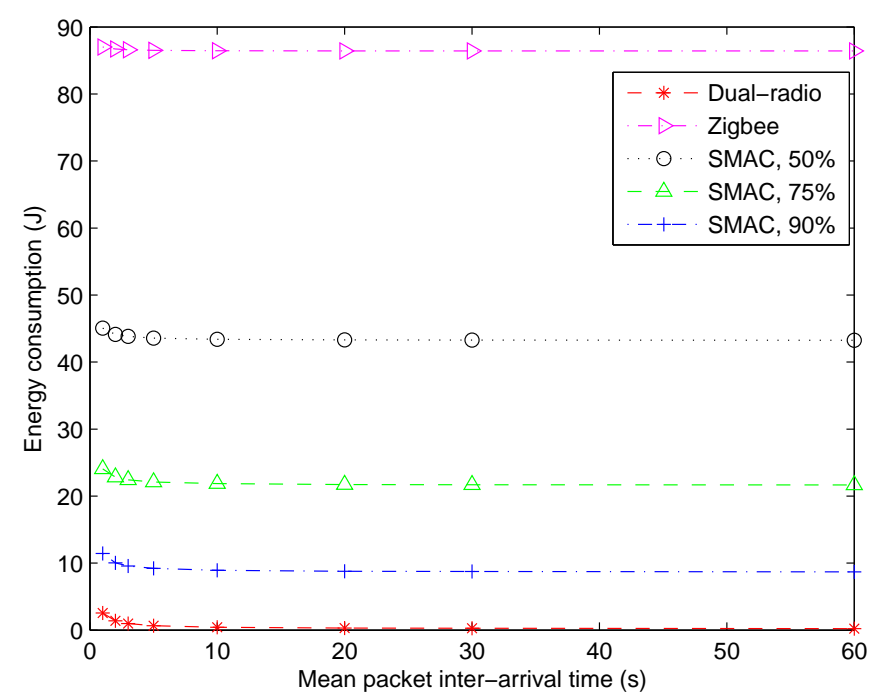

Figure 3-5: Analytical result for node energy consumption (Chipcon cc1000): Using dual-radio node model results in energy saving in comparison to all other considered protocols for Chipcon cc1000. (The values in per cent show the proportion of the total time that the SMAC node is in sleep mode.)

protocol as it's MAC. In Zigbee non-beacon-enabled protocol, no duty cycle is implemented and nodes are always monitoring the channel. This figure also shows the energy consumption of the node working based on SMAC for different sleep periods of 50, 75, and 90 percent. As we see the wake up radio results in significant energy saving in this case for all event rates. The reason of such improvement is that the radio model used for this case (Chipcon cc1000) consumes much more power than the wake up radio in idle, reception, and transmission modes. In other words, the energy consumed in idle listening is much more for the Zigbee and SMAC nodes compared to dual-radio node since Chipcon cc1000 consumes much more power in the idle state than the wake up radio.

Figure 3-6 depicts the results for LP Radio. In this case the difference in the power consumption of the wake up radio and the main radio model is lower. There is an intersection between the energy consumption values of the dual-radio node and the 90 percent sleeping SMAC protocol. This shows that for very high event rates (around 1 event per second and more) the SMAC with 90 percent sleep periods performs better than the dual-radio node in terms of energy efficiency. We have assumed that the SMAC 90 per cent sleeping node handles all these packet transmissions in it's active period. Note that if the switching time of the main radio or the power consumed during the switching increases, the energy required for this 


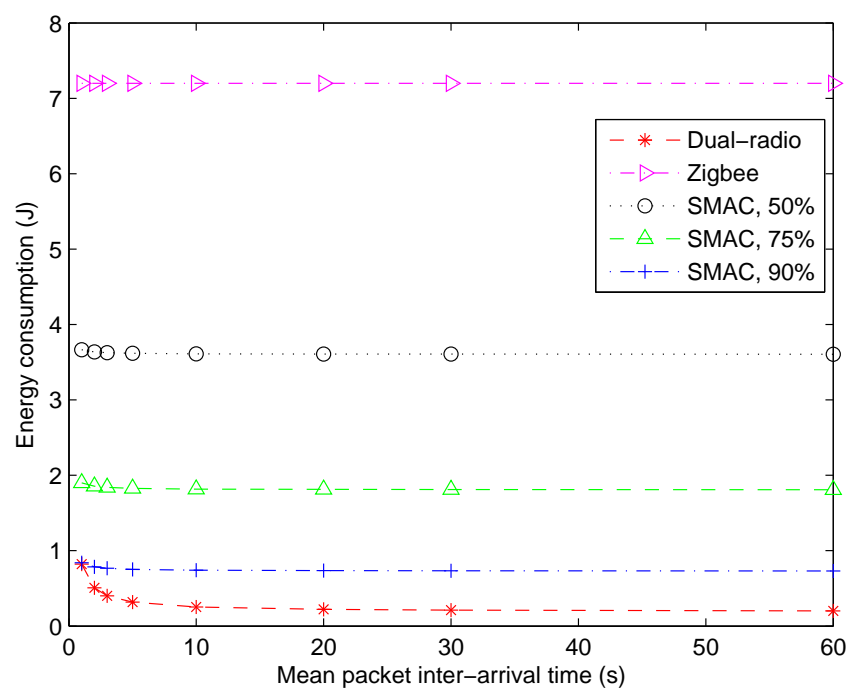

Figure 3-6: Analytical result for node energy consumption (LP Radio): For very highe event rates SMAC with 90 per cent sleep period performs better than dual-radio. In all other cases, dual-radio model consumes less energy. (The values in per cent show the proportion of the total time that the SMAC node is in sleep mode.)

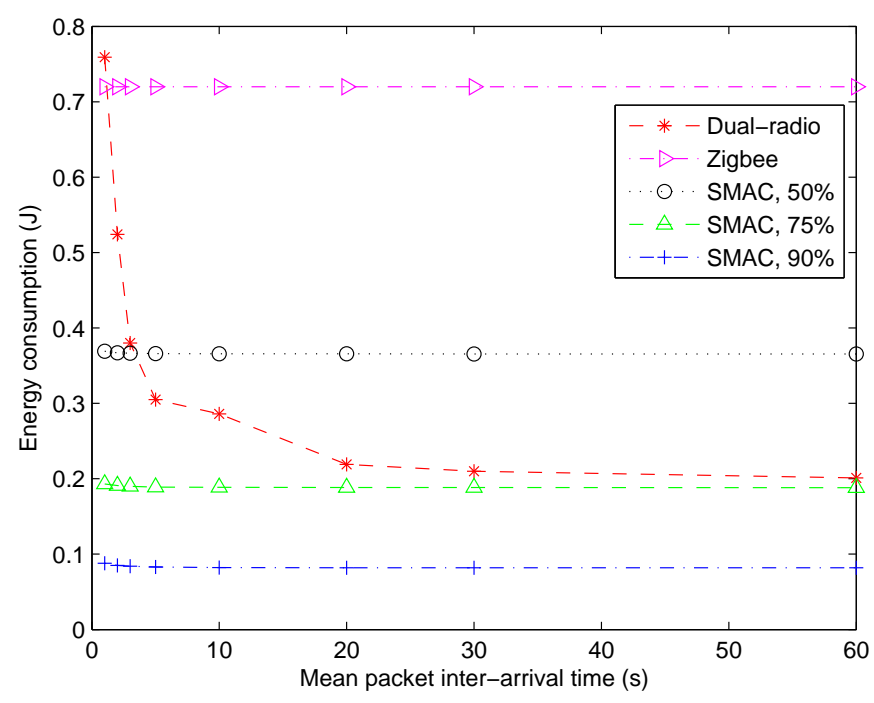

Figure 3-7: Analytical result for node energy consumption (IMEC): Due to low power consumption of IMEC, the dual-radio energy performance degrades. Dual-radio node performs better than the Zigbee node. It also has higher energy efficiency than SMAC with 50 per cent duty cycle for low event rates. SMAC with 75 and 90 per cent sleep periods consume less energy than the dual-radio. (The values in per cent show the proportion of the total time that the SMAC node is in sleep mode.) 
action is increased and the energy performance of the dual-radio node degrades. So, the intersection point occurs in lower event rates when energy consumption of switching increases.

As we see in Figure 3-7 due to the low power consumption of IMEC Radio, the dualradio node consumes more energy than the SMAC with 90 and 75 percent sleeping period. It also consumes more energy than the Zigbee and SMAC with 50 percent sleeping in high event rates. The critical event rate is the rate above which the dual-radio node consumes more energy than the single-radio node. Critical event rate is 51 events per minute for Zigbee and 18 events per minute for SMAC with 50 percent sleeping for the assumed parameters.

As mentioned above, this method for calculating the energy consumption of the wireless node depends on the position of a node in the network and the number of neighbors it has. So, knowledge of the network topology around the node is needed.

The next chapter describes the proposed dual-radio model and explains the results of it's implementation in OPNET. We will compare the simulation results and analytical results, and investigate the dual-radio node performance. 


\section{Chapter 4}

\section{Node Model Description and Results}

\section{4-1 Overview}

In this chapter, we introduce the node model that we designed in OPNET environment and make use of it to investigate the characteristics of the dual-radio system. Our goal is to compare the performance of a network equipped with dual-radio nodes with a Zigbee network in terms of energy efficiency and delay.

In the first section, we describe the node model. In the second section, we discuss the results. The chapter will finish with a section, explaining the dual-radio cooperation.

\section{4-2 Node Model}

We describe the dual-radio model that we designed and used in our simulations and analytical calculations. First, we briefly talk about OPNET and how we implemented the wake up radio model in this software. Note that the dual-radio model has an identity-based wake up receiver which includes the address of the target node in the wake up tone.

OPNET (OPtimized Network Evaluation Tool) is a software designed for performance analysis of networks [54]. Users can analyze simulated networks to compare the impact of different technology designs on end-to-end behavior. It is the fastest discrete event simulation engine. Availability of hundreds of protocols and vendor device models with source code, and realistic application modeling and analysis are other features of this software.

OPNET has a hierarchical modeling environment. Designing the network model, node and link models, and process models are the steps of this hierarchical modeling. The first step is designing a network. The network environment, distance between nodes, node models and link models, packet inter-arrival process and it's mean, 


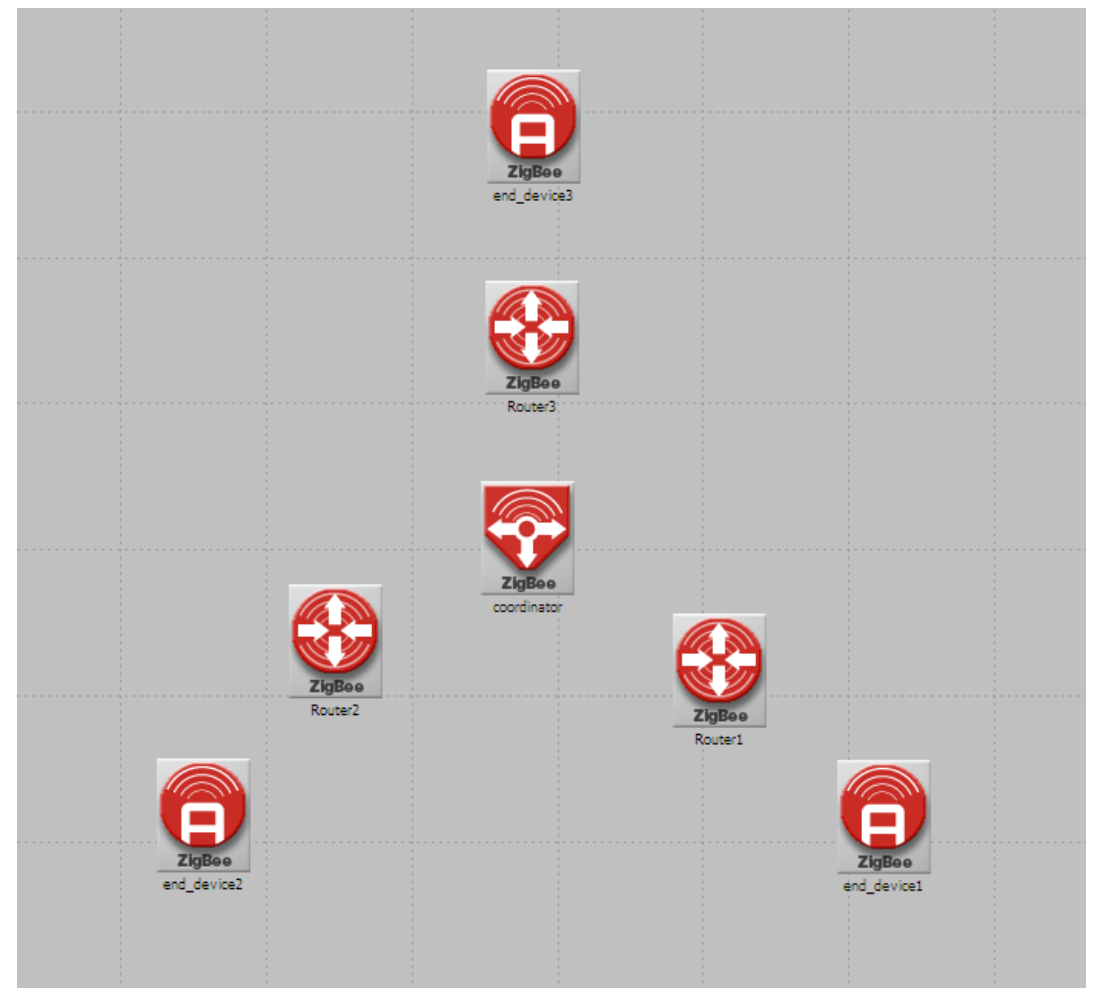

Figure 4-1: Network model: The Zigbee coordinator, three routers, and three end devices make this network in the OPNET environment, which is chosen an office in this case.

and all other network characteristics can be set. There are lots of node and link models in OPNET and you can make a network with the available models simply. If the required link or node models are not available, it is possible to make new link or node models such as dual-radio node model in our case. Each node is made up of different modules which are responsible for the tasks of a specific layer as in OSI model. Each module works based on it's process model which determines the module's tasks and characteristics.

Figure 4-1 shows a simple Zigbee network with three routers and three end devices in OPNET. As mentioned above, it is possible to select different environments such as an office or the world as your network environment. The distance between nodes can be set and the way you connect the nodes may be wireless or wired by choosing various links. Our network is a wireless Zigbee network. The ditances between nodes are chosen in a way that the nodes are within each others' communication range.

In Zigbee, the physical layer uses a certain radio channel according to a specific modulation and several spreading techniques for data transmission and reception [55]. The IEEE 802.15.4-2003 standard offers three unlicensed frequency bands. These bands are $2.4 \mathrm{GHz}$ (worldwide), $915 \mathrm{MHz}$ (North America), and $866 \mathrm{MHz}$ (Europe). There is one channel between 868 and $868.6 \mathrm{MHz}$. The number of channels between 902 and $928 \mathrm{MHz}$ are 10. There are also 16 channels between 2.4 and 2.483.5 GHz. 

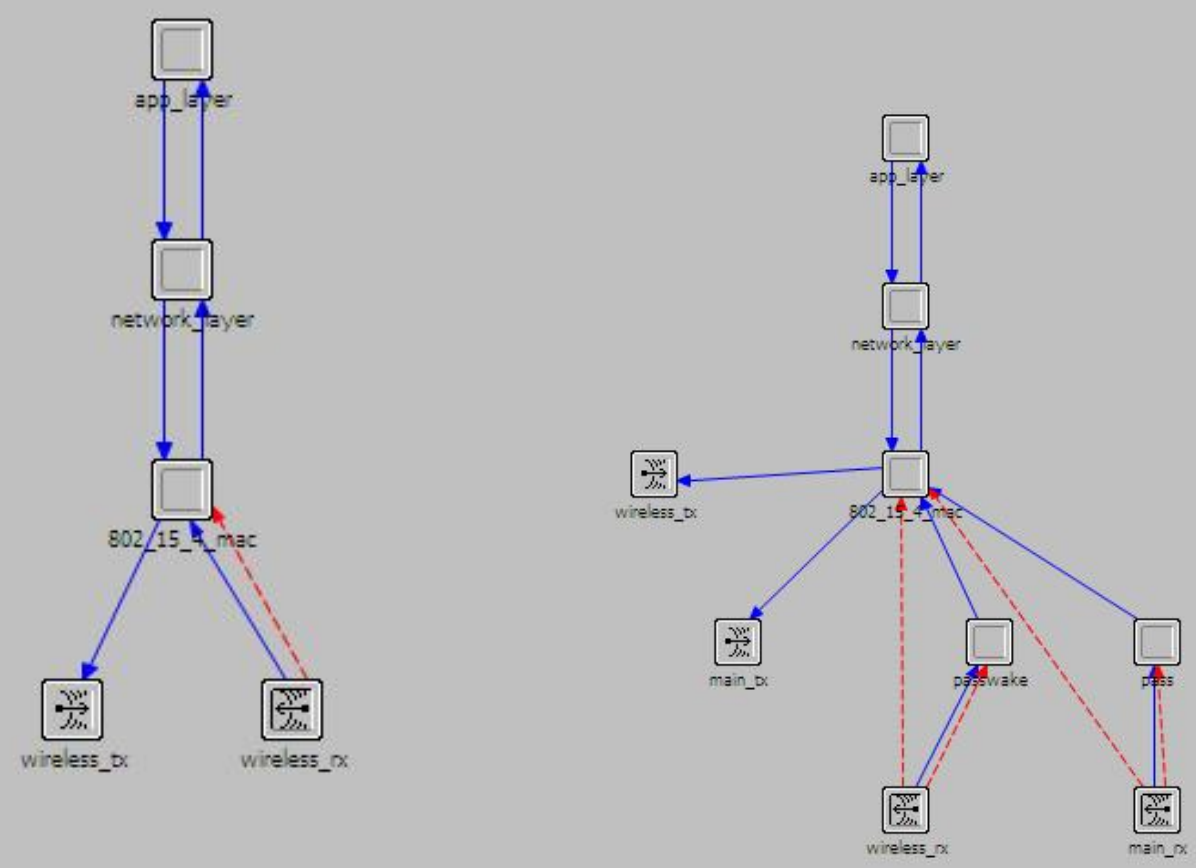

Figure 4-2: Zigbee node model: Three upper modules represent the application, network, and MAC layers, and wireless transmitter and receiver represent the physical layer.
Figure 4-3: Dual-radio node model: Two transmitters and two receivers are added to Zigbee node model in order to make the dualradio node model.

The MAC protocol supports two modes: beacon-enabled mode and non-beaconenabled mode. In beacon-enabled mode, the coordinator periodically generates beacons for synchronization, network identification, and superframe structure description. In non-beacon-enabled mode, nodes send their data by using unslotted CSMA/CA mechanism. Scalability and self-organization are the advantages of nonbeacon-enabled mode while lack of time guarantee to deliver data is it's disadvantage.

Figures 4-2 and 4-3 show the node model of Zigbee and dual-radio node model respectively. We see the wireless transmitter and receiver which make the physical layer of the Zigbee node model. Three other modules represent the MAC, network, and application layers of the node. The physical layer contains the transmission and reception components which are responsible for data exchange. The MAC module provides the functional and procedural means to transfer data and to detect and correct errors occured in the physical layer. The network layer is responsible for 


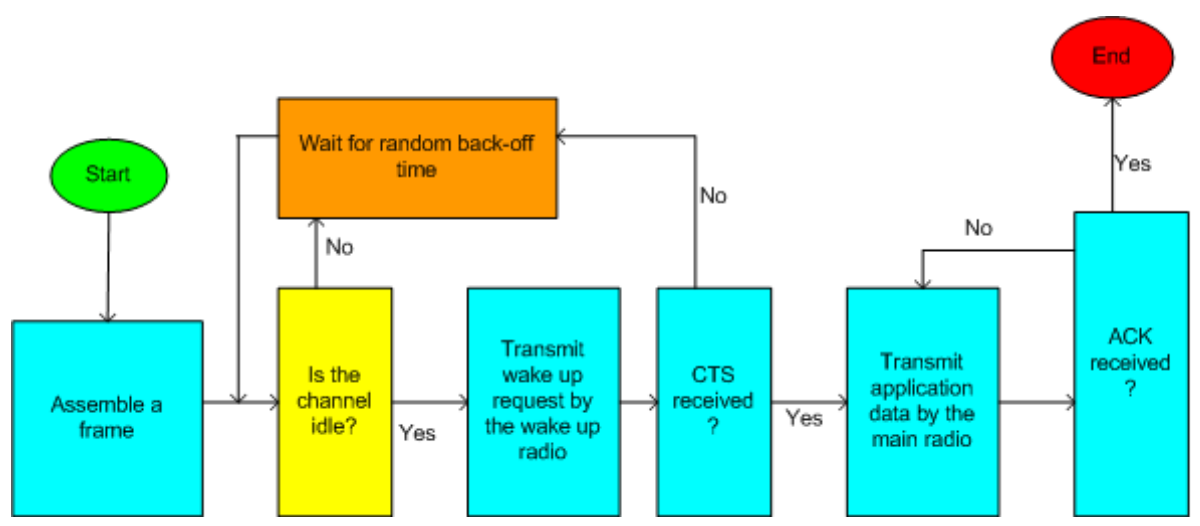

Figure 4-4: Dual-radio MAC block diagram: The dual-radio node invokes the CSMA/CA process for transmission of Wake up Request by the wake up radio, and sends data packet by the main radio after Clear To Send reception. If the transmitter does not receive the Acknowledgement, it will retransmit the data packet.

transferring variable length data, and for routing packets delivery including routing through intermediate routers. Finally, the application layer is responsible for identifying communication partners, determining resource availability, and synchronizing communication.

In order to design the dual-radio node we use the node model of Zigbee end device and add a radio transmitter and receiver to it. So, the dual-radio node has application layer, network layer, and MAC layer modules, and two wireless transmitters and two receivers which represent the wake up and main radio. The two new modules, PASS and PASSWAKE, are used to process the packets received by the main radio and wake up radio, before passing them to the MAC module.

Each of these modules work based on it's process model. In order to change the Zigbee node to dual-radio model, we should change the MAC module which defines it's working principles. The MAC of the Zigbee node works based on CSMA/CA (Carrier Sense Multiple Access with Collision Avoidance). A node which has data to send, invokes the CSMA/CA process. To send a packet, the CSMA/CA process first listens to the channel to check if it is free or not. If the channel appears free, the packet is sent. Otherwise, it waits for a random back off time and starts the process again. Figure 4-4 shows the CSMA/CA algorithm by using a simple block diagram.

For the dual-radio node, we use the CMSA/CA process to send Wake up Request by the wake up radio. When a node detects an event, it invokes the CSMA/CA mechanism. The Wake up Request message is sent by the wake up radio when CS$\mathrm{MA} / \mathrm{CA}$ is done successfully. Once the CSMA/CA process is finished successfully, the node turns it's main radio on.

The receiver of the Wake up Request message may be busy or the wake up message may be destroyed in the channel. In this case, there would be energy waste due to useless turning on of the main radio. On the other hand, if the node waits for the CTS message to turn it's main radio on, there will be increased delay and increased probability of collision of data packets since another node may start a transmission 


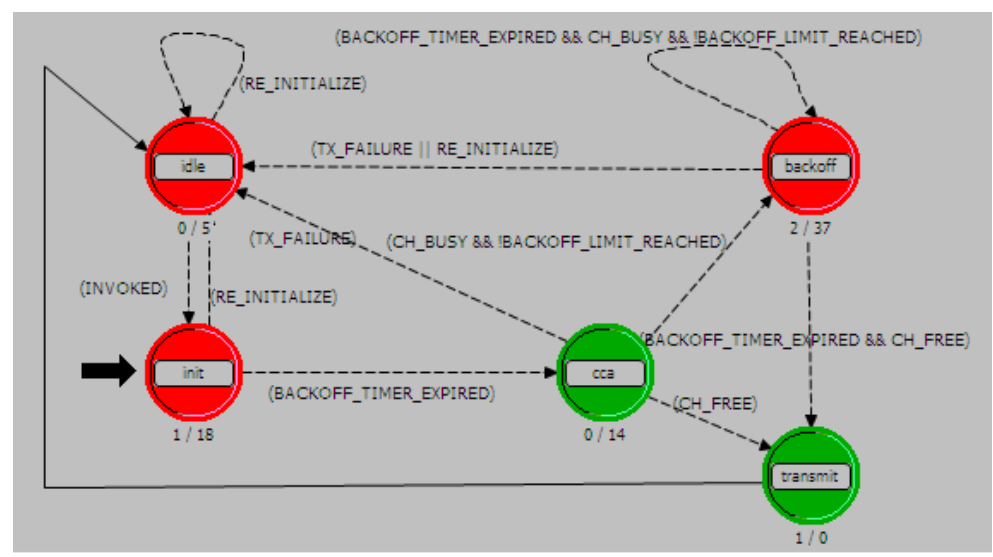

Figure 4-5: CSMA/CA process model: The MAC process model defines the CSMA/CA protocol. Initialization, idle state, channel checking, back-off state, and transmission are the five states defining the CSMA/CA process model.

session during this period. Considering all these, the successful notification of the CSMA/CA process is considered to be the best time of triggering the main radio on.

If the receiver of the Wake up Request message is not busy, it sends a message back to the sender indicating that it has received the Wake up Request, and it is waiting for the data packet on the main radio. At this time, it turns it's main radio from sleep mode to active state.

When the transmitter receives the CTS message, it sends the data packet by it's main radio. The session finishes with an acknowledgement from the receiver.

The CSMA/CA process model in OPNET Zigbee node MAC module is used as a child process. In fact, the MAC module of the Zigbee node has two process models: the main process and the CSMA/CA process which is called when needed.

Figure 4-5 shows the process model of CSMA/CA mechanism, which is used as a child process for the Zigbee protocol and dual-radio model. It is invoked by the Zigbee model to send data, and by the dual-radio model to send Wake up Request. It consists of five states which define the CSMA/CA process at each moment. The initial state is responsible for initializing the variables. The idle state is the default mode of the process and the state that the process goes to, after each transmission. When the CSMA/CA is invoked, the process goes from the idle state to the initial state for the initialization of the variables. The CCA state checks if the channel is free. If it finds the channel free, it sends the packet by going to the transmit state. Otherwise, it goes to the back-off state and stays there until the determined back-off time is finished.

The transitions that connect the states define the conditions for going from one state to another. These conditions and the tasks that should be done when they happen are defined in the process model's function block.

A green (forced) state is a state that the process goes to another state when the functions of this state are done. In contrast, the process remains in the red (un- 


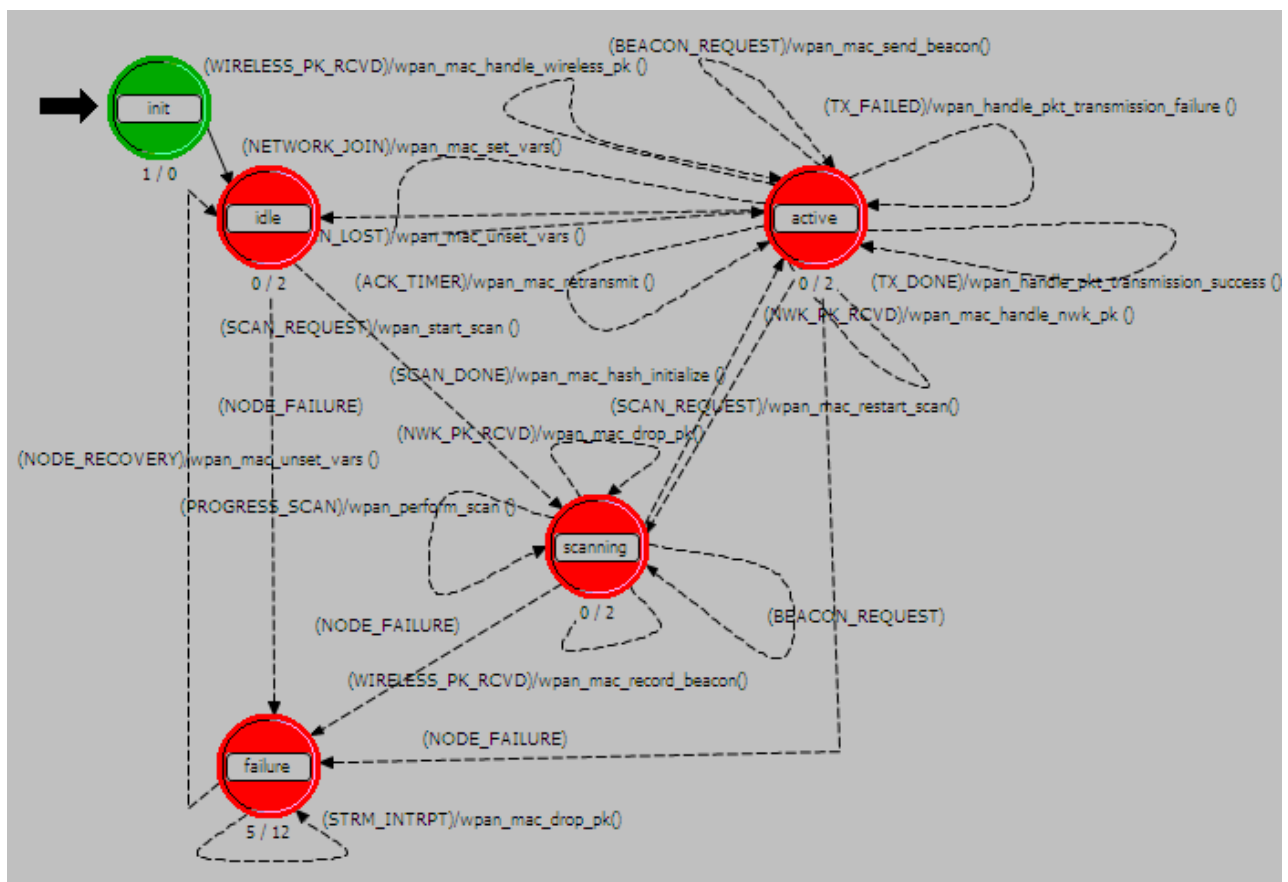

Figure 4-6: Zigbee MAC process model: Zigbee MAC module is made of a main process model which invokes the CSMA/CA as a child process whenever it intends to transmit data. Init state initializes the variables. The node is in active, scanning, and idle state when it is involved in transmission, channel detection, and idle mode respectively. Failure state is for errors and node failure.

forced) state, after finishing their tasks, until an instruction comes or an interrupt occurs indicating that the process should go to another state. For instance, in the CSMA/CA process model, the CCA state is a green state since after listening to channel, the process either goes to transmit state or back-off state automatically dependent on the result of the CCA state. But, it will remain in the idle state, as a red state, until an interrupt comes indicating that the process is invoked and there is a packet to send.

Figure 4-6 shows the main process model of the MAC module of Zigbee protocol. Like CSMA/CA, the initial state is responsible for initializing the variables. The failure state is responsible for the case of node failure which happens due to an error. Idle state represents the node state when it is idle. Active state is when the node is involved in a data exchange session. The scanning state is responsible for detecting the channels.

The MAC process model of the dual-radio node is the same as the Zigbee protocol. It only adds one new transition from the active state to itself for the Wake up Request timer. The whole changes are implemented in the function block of the MAC module. Now we discuss the features we have implemented in the dual-radio model to optimize it's performance. 


\section{4-2-1 Network Allocation Vector}

We add some features to the simple MAC protocol of the Zigbee node to improve it's performance. First, we define a section in the Wake up Request and CTS messages that contain the time that the channel will be occupied by the ongoing transmission. Hence, these two messages contain the transmitter and receiver address and the time that the transmission takes. This part of the Wake up Request and CTS messages is called Network Allocation Vector (NAV). The sender computes the NAV as the time required to receive CTS and send data by dividing the size of these packets by data rate of the radio which sends them. The receiver of the data uses the same method to compute the NAV of the CTS message. All nodes are monitoring the channel by their wake up radios continuously. Once they receive a Wake up Request destined for another node, they check the NAV and refrain from transmitting any packets until the NAV time of their neighbors is finished. This method decreases the number of collisions, which is a major source of energy waste and delay.

If a node intends to transmit more than one data packet, it can compute the NAV as the time required for transmission of all the packets. This method can be used so that the extra Wake up Request and CTS messages for each transmission are eliminated and the communication delay is decreased.

\section{4-2-2 Busy Mode}

Defining a Busy state is another feature of the designed dual-radio MAC. Each node which starts a transmission session, goes to a Busy state. If another packet comes from the application layer during this transmission state, the node queues this packet. After the data transmission, the transmitter checks it's queue to see whether there are any packets awaiting to be sent. If it finds one, it invokes the CSMA/CA process to send Wake up Request. The Busy mode is unset when the transmission session is done successfully. In order to prevent the node from staying in the Busy state due to packet lost, the Busy state is unset after the time required for a complete transmission of wake up, CTS, and data messages.

\section{4-2-3 Timers}

We define timers for both Wake up Request and data messages. When the CSMA/CA process sends the Wake up Request message successfully, a timer starts. If after the defined duration the CTS is not received, the CSMA/CA is invoked again to retransmit the Wake up Request.

There is another timer for the transmitter defined to ensure that the destined node receives the data packet. When a node sends data it starts a timer. Once the timer is expired and the acknowledgement is not received, the node retransmits the data packet.

Next section describes the simulation results. 


\section{4-3 Results}

In this section, we investigate the results of the tests and compare them with the analytical results discussed in the previous chapter. Setting different attributes for nodes and networks is possible in OPNET. As explained before, there are numerous existing node and link models in OPNET and we can design new models as well. Node attributes such as the application layer data packet size, the distribution and mean of the packet inter-arrival time, the destination of the packets, the number of children of a coordinator in a Zigbee network, the MAC parameters like the maximum number of back offs, and the back off exponent can be set.

There are many different parameters which affect the results. The energy consumption of the dual-radio node when switching from sleep mode to idle mode is one of the most important parameters in determining the energy efficiency of the wake up radio. The duty cycle of the Zigbee node has a significant effect on the trade-off between energy consumption and delay in the Zigbee network. The size of the packets and the data rate of the radios also affect the results. They have influence on the number of collisions, media access delay, and end-to-end delay. They determine the time that the radio spends in each state and affect the energy consumption of each node.

We used three radio types introduced in Tables 3-1, 3-3, and 3-3 as the main radio models. Simulation parameters are the same as what mentioned in Table 3-4. Again we do not take the ACK messages into account.

First, we focus on the energy efficiency issue.

\section{4-3-1 Energy Consumption}

We deploy the simple network in Figure 4-1 to make simulations in order to see the energy consumption of an end device. We test this network for Zigbee nodes and dual-radio nodes equipped with the radios which were introduced in Tables 3-1, 3-2, and 3-3. The packet inter-arrival time has exponential distribution. The data rate of the wake up radio is $1 \mathrm{kbps}$ and the size of the data packet is assumed 1024 bits. The confidence interval of the simulation results is depicted for each figure. This interval is computed as twice the standard deviation of the simulation results. The number of simulations for each individual node is 30 .

Figure 4-7 shows the energy consumption of a Zigbee end device in a one-hour period resulted from simulation and analytical model. The radio model used is Chipcon cc 1000. Figure 4-8 shows the result of the same simulation for a dual-radio node whose main radio is a Chipcon cc1000 radio. The simulation and analytical results are close to each other. The energy consumption of the dual-radio node is much less than the Zigbee node due to high power consumption of the Chipcon cc1000 radio in idle, reception and transmission modes in comparison to $50 \mu \mathrm{W}$ power consumption of the wake up radio.

The results show that for all assumed event rates, the energy saved compared to single-radio model, is more than 97 per cent. Therefore, if the main radio model 


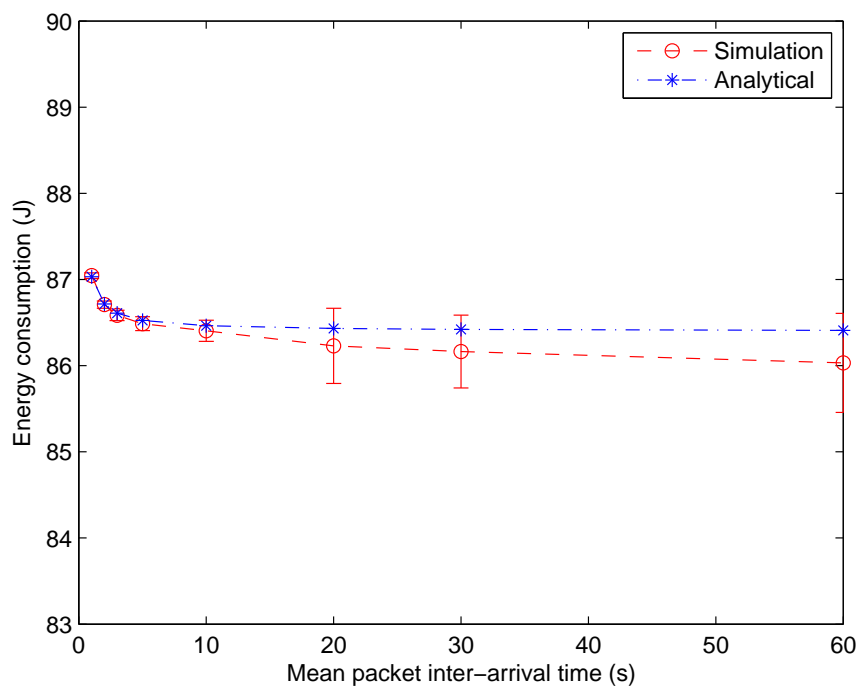

Figure 4-7: Zigbee node energy consumption (Chipcon cc1000): Large energy consumption is a result of large power consumption of the radio model in idle listening mode.

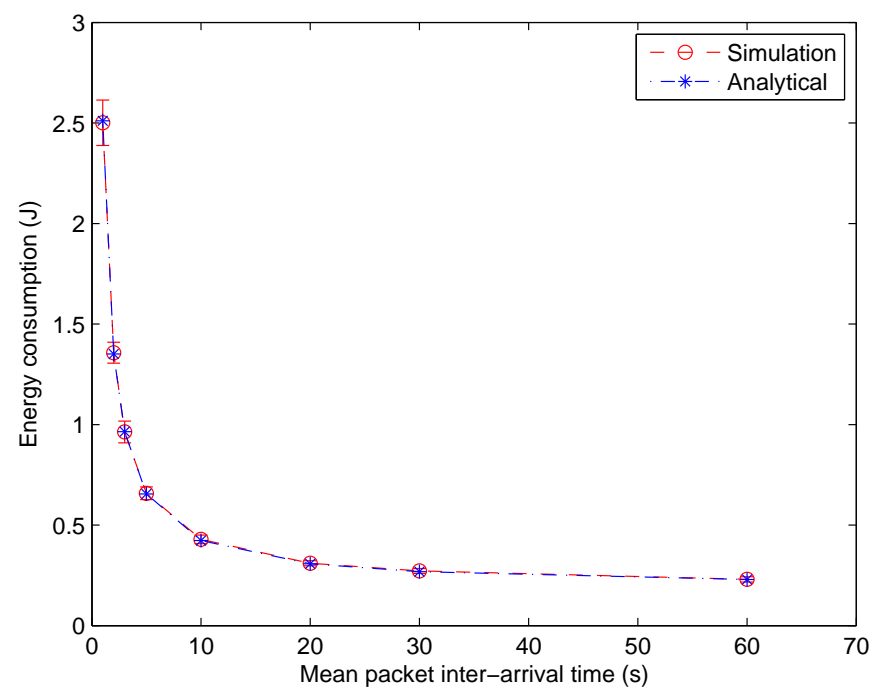

Figure 4-8: Dual-radio node energy consumption (Chipcon cc1000): Compared to the Zigbee node, tremendous energy saving (more than 97 per cent for all event rates) is achieved. This is a result of low power consumption of the wake up radio in idle listening in contrast to Zigbee node. 


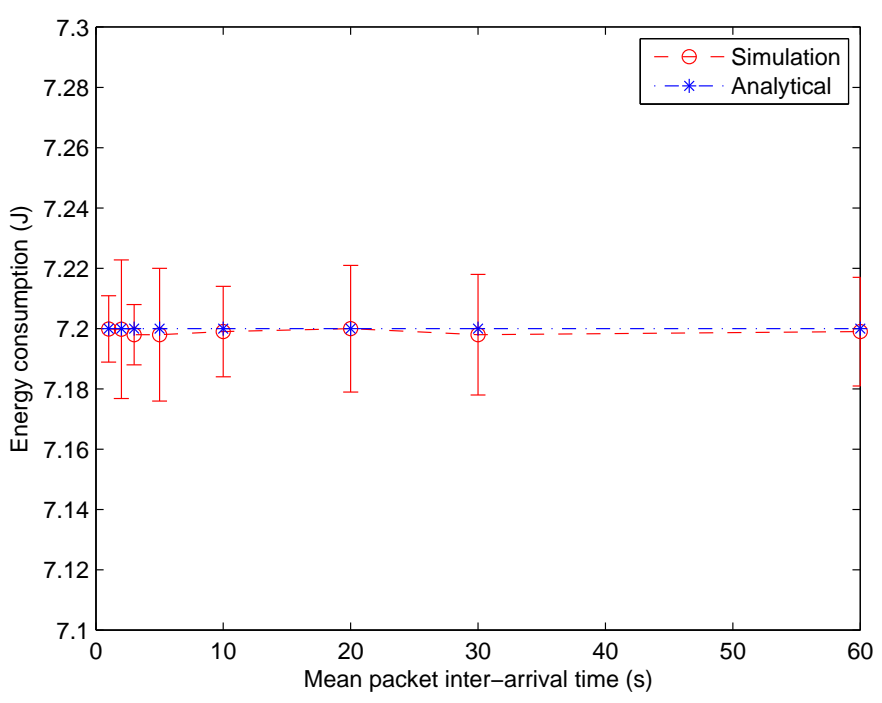

Figure 4-9: Zigbee node energy consumption (LP Radio): Energy consumption for different event rates is the same since we assumed that LP Radio has the same power consumption in transmission, reception, and idle state.

has much higher power consumption in comparison to the wake up radio, using the dual-radio node model decreases the node's energy consumption significantly.

Figures 4-9 and 4-10 show the same results for LP Radio. The Zigbee node is expected to consume the same energy for different event rates since LP Radio consumes the same power during Transmission, reception, and idle states and it does not have a sleep schedule. We see much better performance when the dual-radio node is used. However, the decrease in energy consumption is not the same as the case where we used Chipcon cc 1000, because LP Radio has less power consumption than the Chipcon cc1000.

The decrease in energy consumption is approximately 88 per cent for the largest assumed event rate, and 97 per cent for low event rates. This shows that using the wake up radio saves more energy in low event rates since the wake up radio keeps the main radio inactive when the channel is idle and wakes the main radio up only when it should transmit or receive data packets.

The next two Figures, 4-11 and 4-12, show the results for the case that we use IMEC as the main radio. The power consumption is the same for different states for this radio model. So, we expect the same energy consumption for various event rates. Since IMEC radio has low power consumption, we see that the energy consumption of the Zigbee node is close to the dual-radio node. For very high event rates the dual-radio node performs worse than Zigbee.

For large event rates, the performance of two radio models is close to each other. We see that for the event rate of one per second, the energy consumption of the single-radio Zigbee node is less than the dual-radio model. For low event rates the energy saving achieved by using the wake up radio is around 72 per cent. 


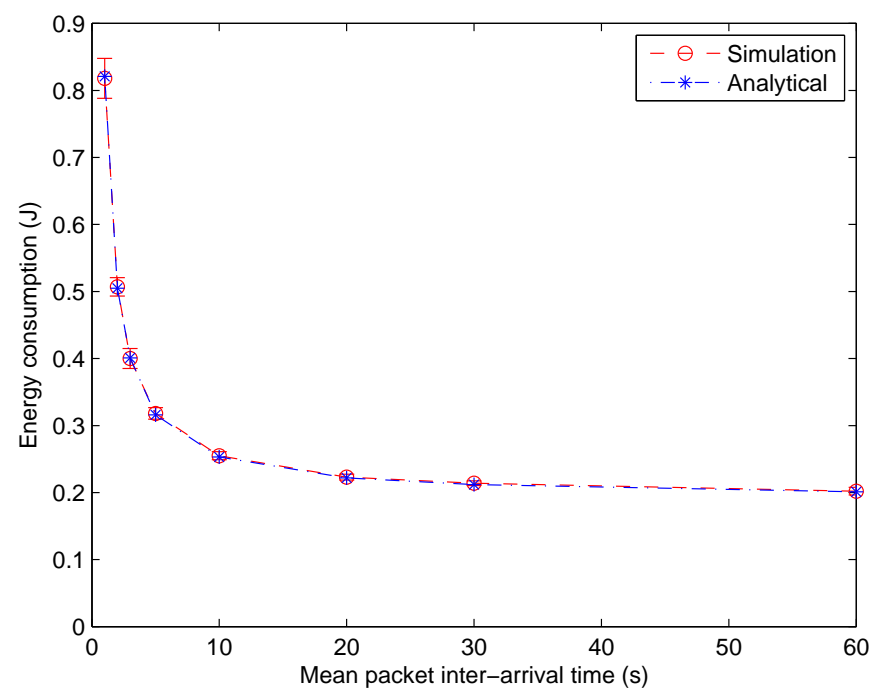

Figure 4-10: Dual-radio node energy consumption (LP Radio): Less energy consumption than the Zigbee node with the same radio model and good match between simulation and analytical results are deduced. Decrease in energy consumption is from 88 to 97 per cent for large to low event rates. Improvement in energy efficiency is larger for low event rates since the wake up radio eliminates the time that the main radio consumes a lot of energy, listening to the idle channel.

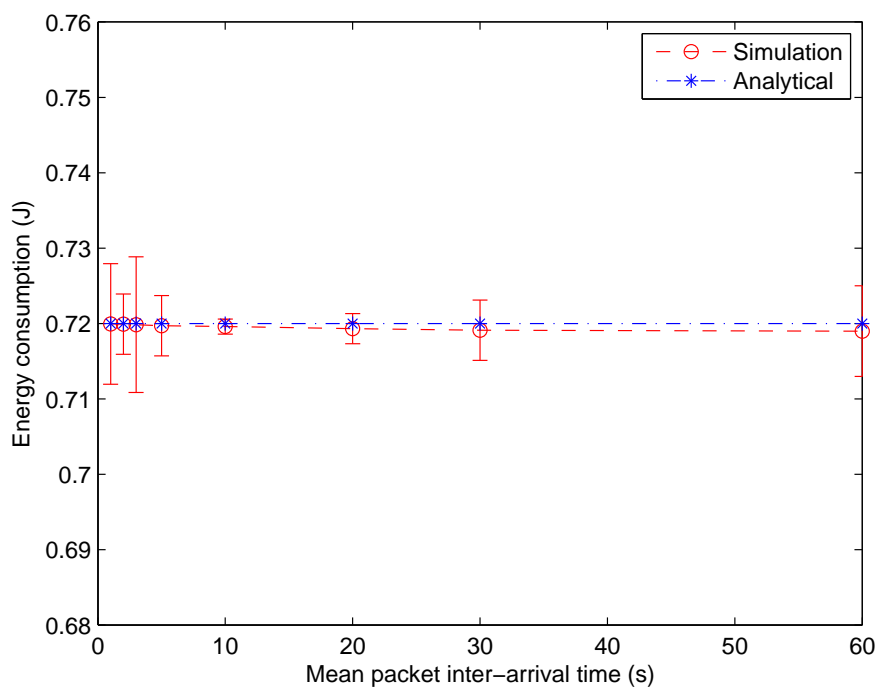

Figure 4-11: Zigbee node energy consumption (IMEC): Low energy consumption of the Zigbee node in comparison to the previous cases with LP and cc1000, is a result of low power consumption of IMEC. 


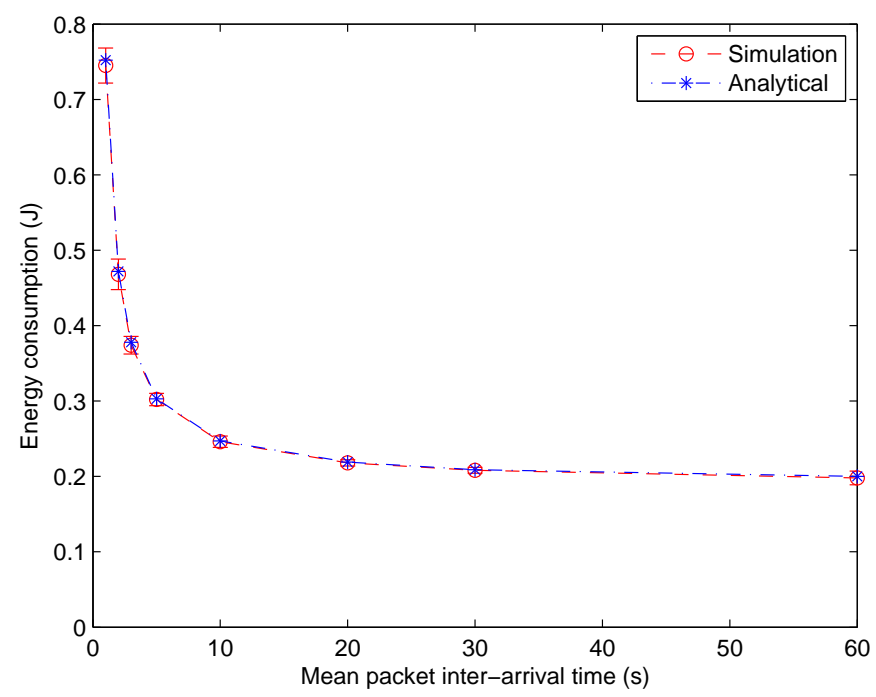

Figure 4-12: Dual-radio node energy consumption (IMEC): In very high event rates, dual radio model consumes more energy than the Zigbee node when the main radio model is IMEC. 72 per cent energy saving is achieved for low event rates compared to the single-radio model.

\section{Switching Energy}

We investigate the effect of the switching energy on the energy performance of the dual-radio node for different radio models. The switching energy is the energy required for turning the main radio from sleep to active state and vice versa. If the dual-radio uses internal energy source for triggering the main radio, the wake up circuitry takes the required switching energy from the power source. In the previous simulations, the switching energy was assumed to be $77.5 \mu \mathrm{J}$. In [56] a wake up circuitry is described for the wake up radio which consumes $217.5 \mu \mathrm{J}$ for each transition. We investigate the results for a reasonable range of switching energy from $77.5 \mu \mathrm{J}$ to $550 \mu \mathrm{J}$. Note that some methods extract the energy from the radio signals for generating wake up signals [56]. So, they do not use the internal power supply for this operation. In our investigation, we assume that this energy is consumed from the internal power source.

Figures 4-13 and 4-14 show the energy consumption of the dual-radio node when the main radio model is Chipcon cc1000 and the switching energy is $250 \mu \mathrm{J}$ and 550 $\mu J$ respectively. Comparing these figures with Figure 4-7, we see that the energy efficiency of the dual-radio node is much higher than the Zigbee node. Although the switching energy is increased compared to the case in Figure 4-8, the large energy consumption of the Zigbee node due to high power consumption of the main radio model, makes the performance of the two models different. The small increase in the total energy consumption, resulted from the increased switching energy, does not affect the dual-radio performance tremendously compared to Zigbee node.

When the switching energy is changed, the energy consumption of the node in high event rates is affected more. This is due to large number of switching the main radio on and off in high event rates. 


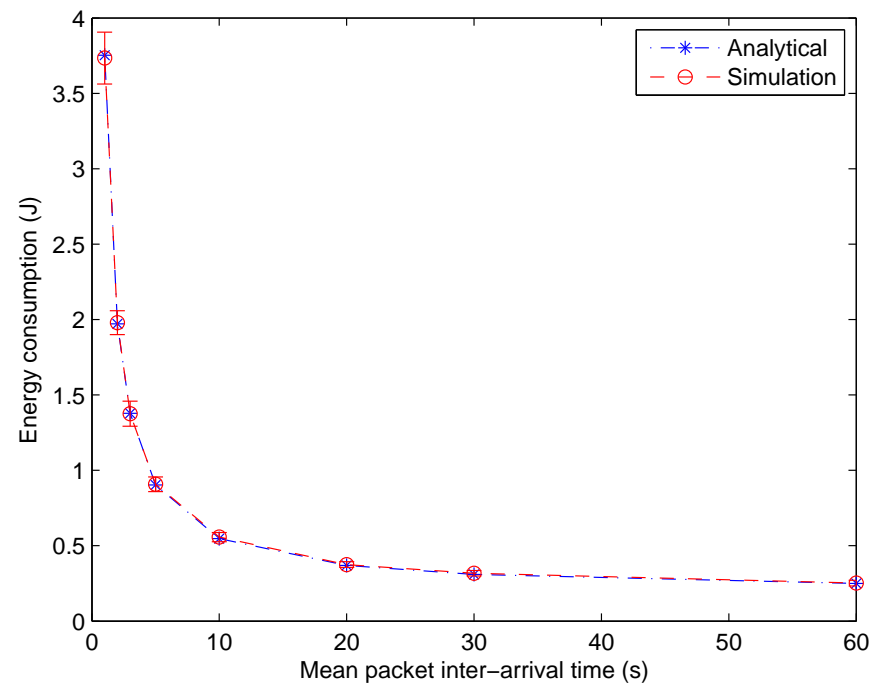

Figure 4-13: Dual-radio node energy consumption for $250 \mu J$ switching energy (Chipcon cc1000): The energy consumption is increased due to increase in switching energy, but is still much less than the Zigbee node. Improvement in energy efficiency is less than the dual-radio with $77.5 \mu \mathrm{J}$ switching energy for large event rates.

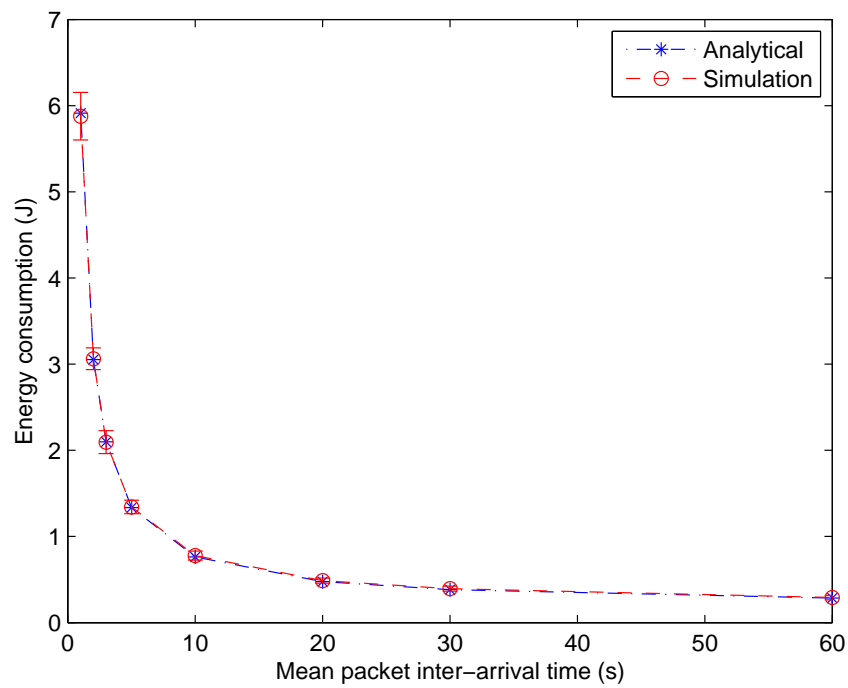

Figure 4-14: Dual-radio node energy consumption for $550 \mu J$ switching energy (Chipcon cc1000): When the switching energy is increased, the increase in node energy consumption is more for high event rates since larger number of switching is done. 


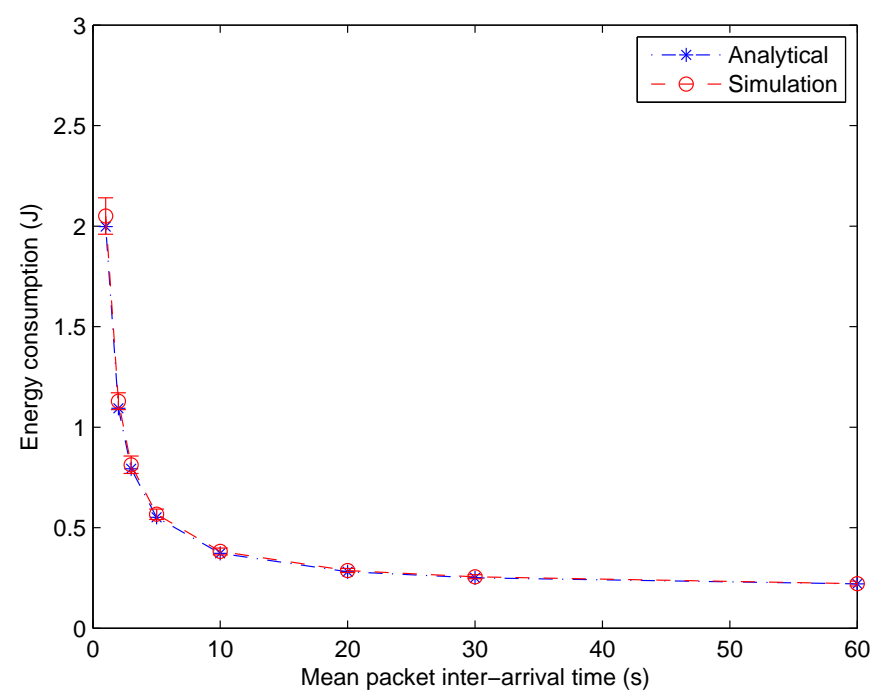

Figure 4-15: Dual-radio node energy consumption for $250 \mu \mathrm{J}$ switching energy (LP Radio): The increase in energy consumption, especially in high event rates, is a result of increase in switching energy. However, the dual-radio still performs better than the single-radio Zigbee node.

When the switching energy is increased to $250 \mu \mathrm{J}$, energy saved by using the wake up radio reduces from 97 to 95 per cent in large event rate of one event per second. For $550 \mu \mathrm{J}$, energy saved in comparison to single-radio is 93 per cent for this event rate. Energy consumption of the node in low event rates is not affected so much.

Figures 4-15 and 4-16 depict the results of LP Radio for increased switching energy. Since the difference between the energy consumption of the dual-radio and singleradio is less for LP Radio in comparison to Chipcon cc1000, increasing the transition energy makes the performance of the two radios close to each other. However, still for all logical values of switching energy, dual-radio consumes less energy than Zigbee single-radio model.

The improvement in energy efficiency for dual-radio node with $250 \mu \mathrm{J}$ switching energy is 72 per cent compared to Zigbee, for the average event rate of one per second. This value is 16 per cent when the switching energy is $550 \mu \mathrm{J}$.

As mentioned before, we assume that IMEC has a constant power consumption of $200 \mu W$. So, when we use this model as the main radio, dual-radio and single-radio has close energy consumption values. Because of this reason, we test the energy consumption of nodes equipped with IMEC for more values of switching energy.

Figures 4-17, 4-18, 4-19, 4-20, and 4-21 show the results. The figures are depicted separately so that the comparison between simulation and analytical results is simple and the confidence intervals are clear. Increasing the switching energy results in degraded performance of the dual-radio node. The increase in node energy consumption is more for high event rates. However, for low event rates, dual-radio model has better energy performance for all assumed values of switching energy. 


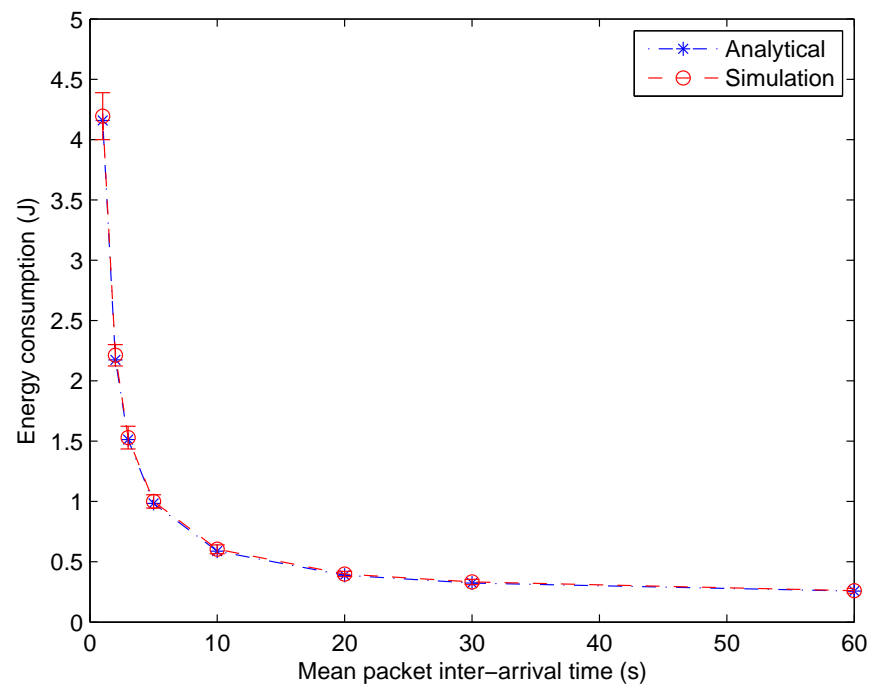

Figure 4-16: Dual-radio node energy consumption for $550 \mu \mathrm{J}$ switching energy (LP Radio): The dual-radio energy consumption gets close to the Zigbee energy consumption, which is $7.2 \mathrm{~J}$ for LP Radio.

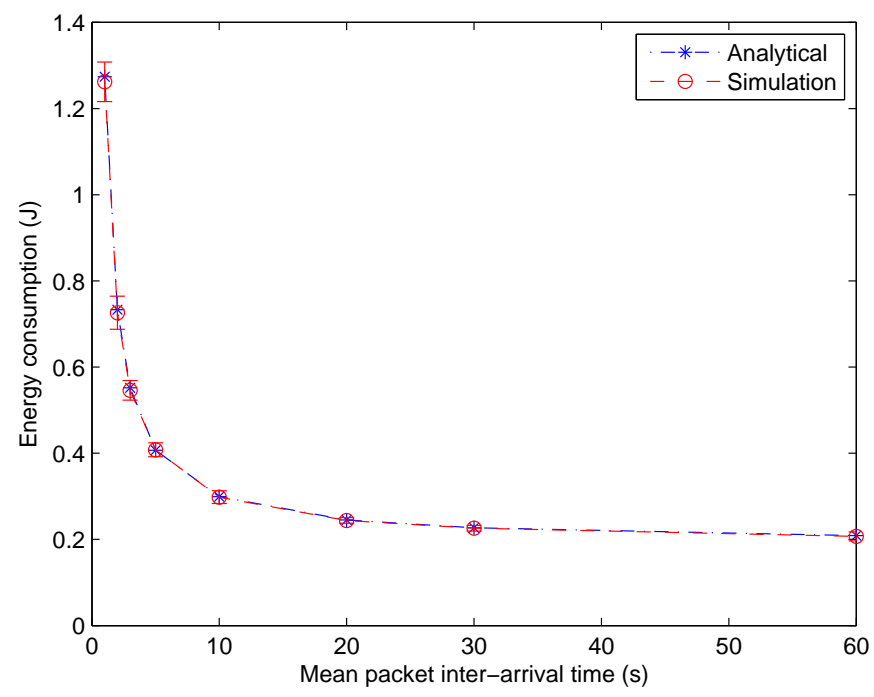

Figure 4-17: Dual-radio node energy consumption for $150 \mu J$ switching energy (IMEC): When IMEC is used as the main radio model, dual-radio node consumes more energy than the Zigbee node for high event rates. Increasing the switching energy decreases the critical event rate, above which the single-radio model performs better. 


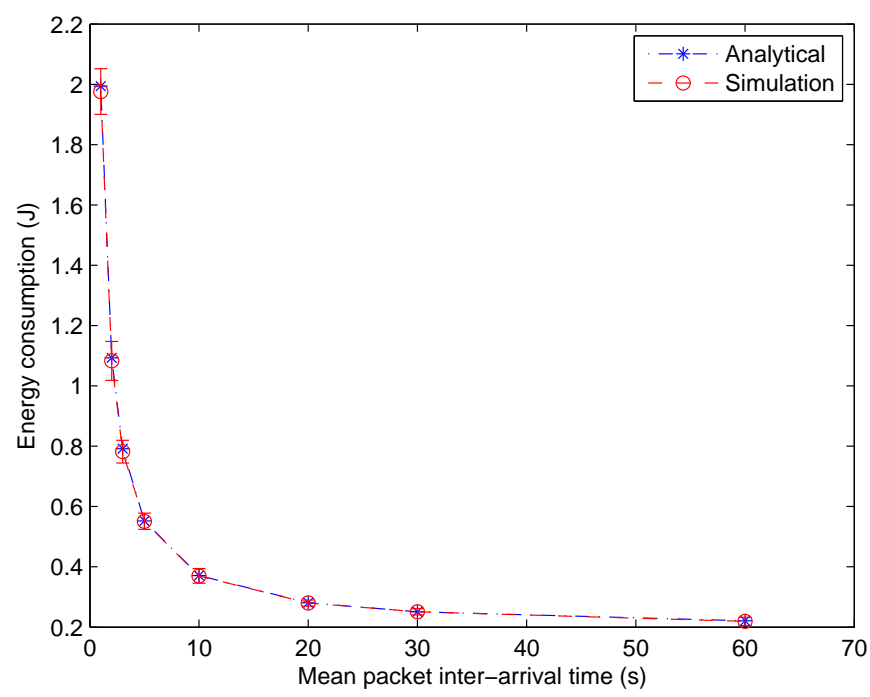

Figure 4-18: Dual-radio node energy consumption for $250 \mu \mathrm{J}$ switching energy (IMEC): Increasing the switching energy shifts the critical event rate to smaller values. For low event rates, dual-radio node consumes less energy than the Zigbee node although the switching energy is increased.

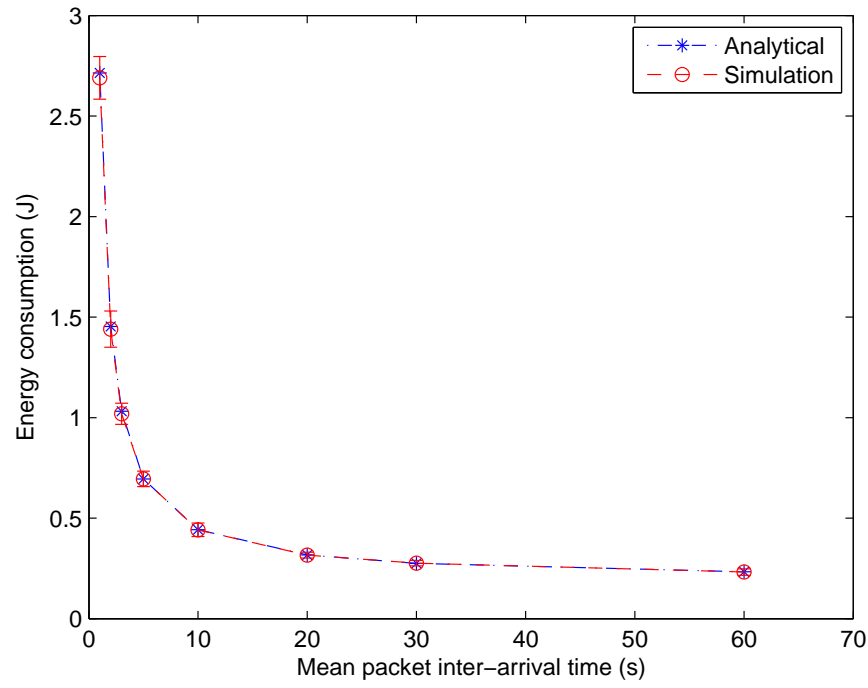

Figure 4-19: Dual-radio node energy consumption for $350 \mu \mathrm{J}$ switching energy (IMEC): Increasing the switching energy affects the energy consumption of the node in high event rates more. 


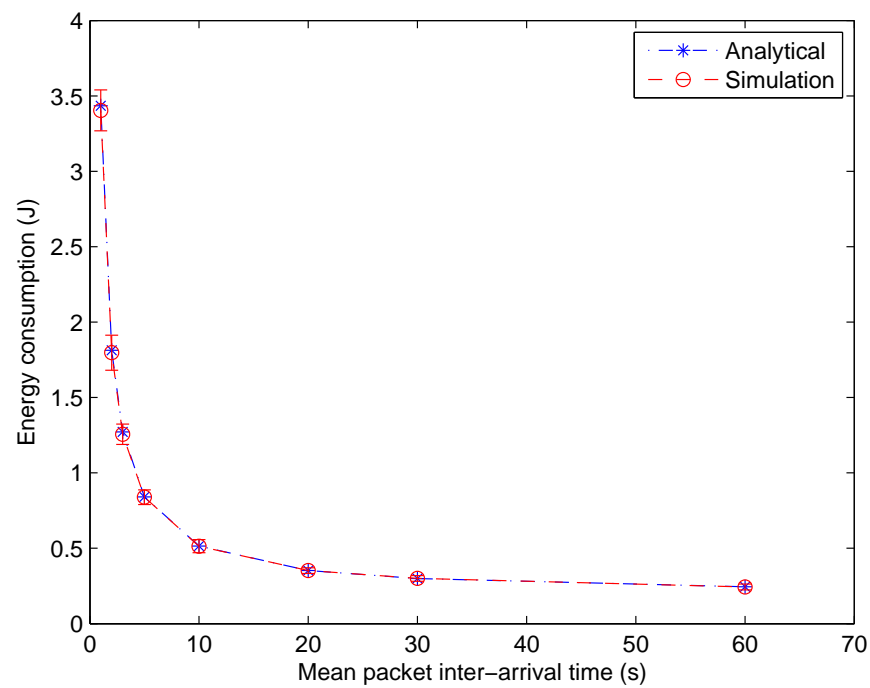

Figure 4-20: Dual-radio node energy consumption for $450 \mu J$ switching energy (IMEC): The critical event rate is around 10 events per minute for $450 \mu \mathrm{J}$ switching energy.

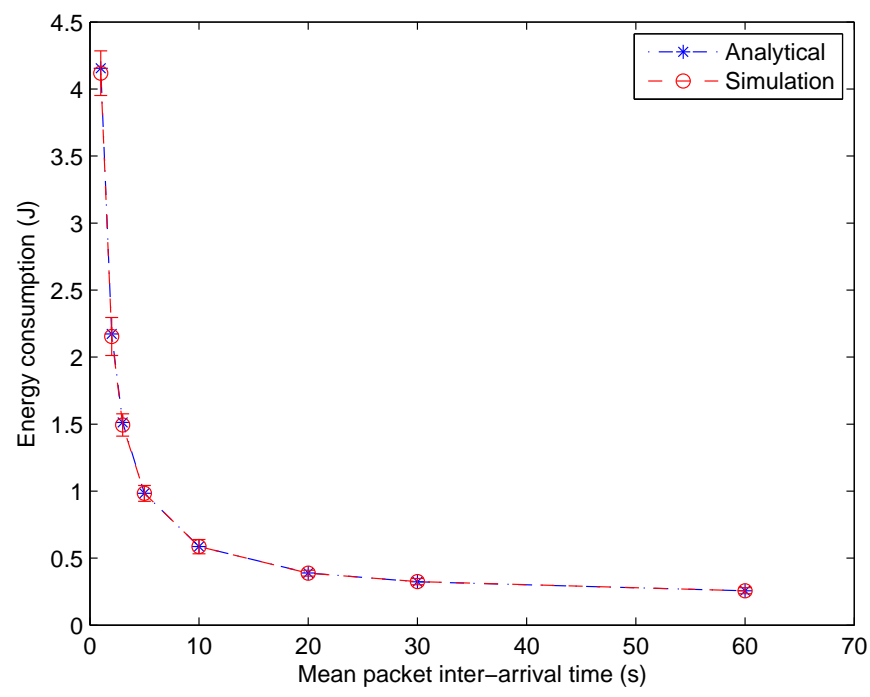

Figure 4-21: Dual-radio node energy consumption for $550 \mu \mathrm{J}$ switching energy (IMEC): For all logical values of switching energy, dual-radio node model results in energy saving in low event rates. 


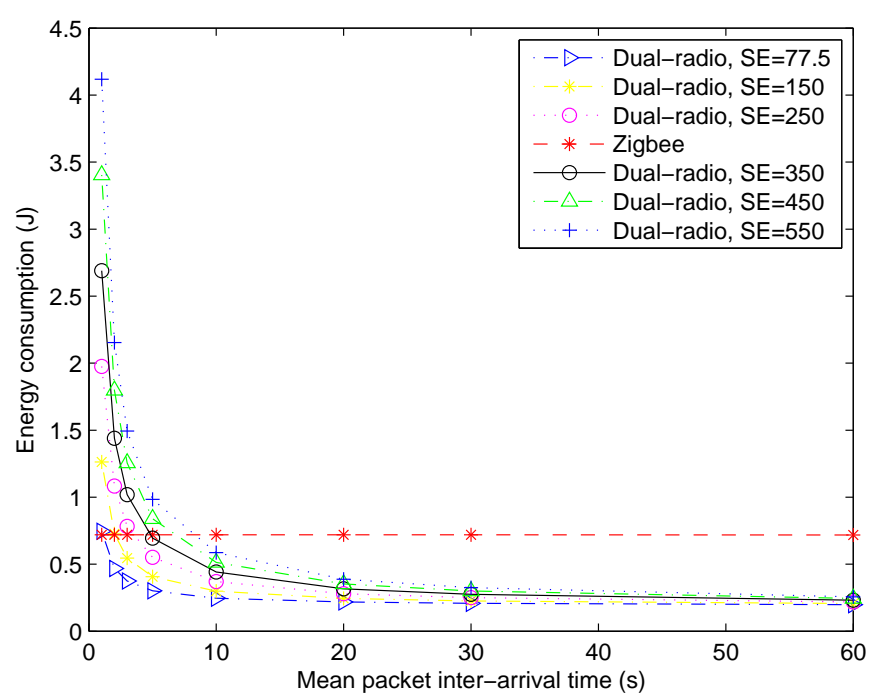

Figure 4-22: Node energy consumption for different Transition Energies using IMEC (simulation): $\mathrm{SE}$ is the Switching Energy and the values mentioned for SE are in $\mu J$. The intersection between the dual-radio and single-radio energy consumption represents the critical event rate resulted from simulation.

\section{Critical Event Rate}

Figures 4-22 and 4-23 sums up the previous results to show the intersection between dual-radio and single-radio node energy consumption for simulation and analytical calculation. The critical event rate is defined as the rate above which the singleradio model consumes less energy than the dual-radio node. The intersection point of energy values of these two models represents the critical event rate.

Considering the results, the switching energy has a significant effect on critical event rate. Again the results of mathematical calculation and simulation are very close to each other. We compute the critical event rate by detecting the intersection point of Zigbee energy consumption and dual-radio node for both simulation and analytical model and plot them in Figure 4-24. The critical event rate decreases as the switching energy increases. As an example, the critical event rate for switching energy of $77.5 \mu \mathrm{J}$ is around 55 events per minute. In other words, if nearly 1 event per second happens, the energy consumption of the dual-radio will become larger than the single-radio node.

If we assume a very small value for the switching energy, or consider the case in which the wake up radio extracts the energy of the radio signals for triggering the main radio, we still can find a critical event rate for the dual-radio model. This critical event rate is not related to energy efficiency. For very high event rates, dependent on the radios' data rate and packet sizes, it may be better to use the single-radio node model. Since a transmission session of dual-radio node takes more time than a Zigbee node, the maximum number of packets sent in the unit of time is less for the dual-radio model. Therefore, using the single-radio model may result in larger data exchange in the unit of time. In conclusion, defining the critical event 


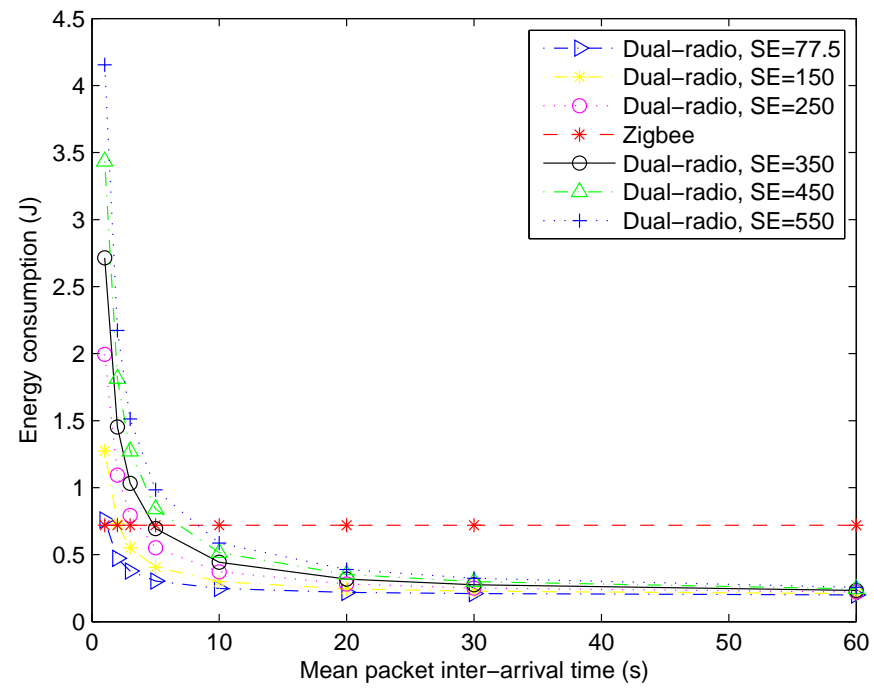

Figure 4-23: Node energy consumption for different Transition Energies using IMEC (analytical): $\mathrm{SE}$ is the Switching Energy and the values for SE are in $\mu J$. The intersection between the dual-radio and single-radio energy consumption represents the critical event rate resulted from analytical model.

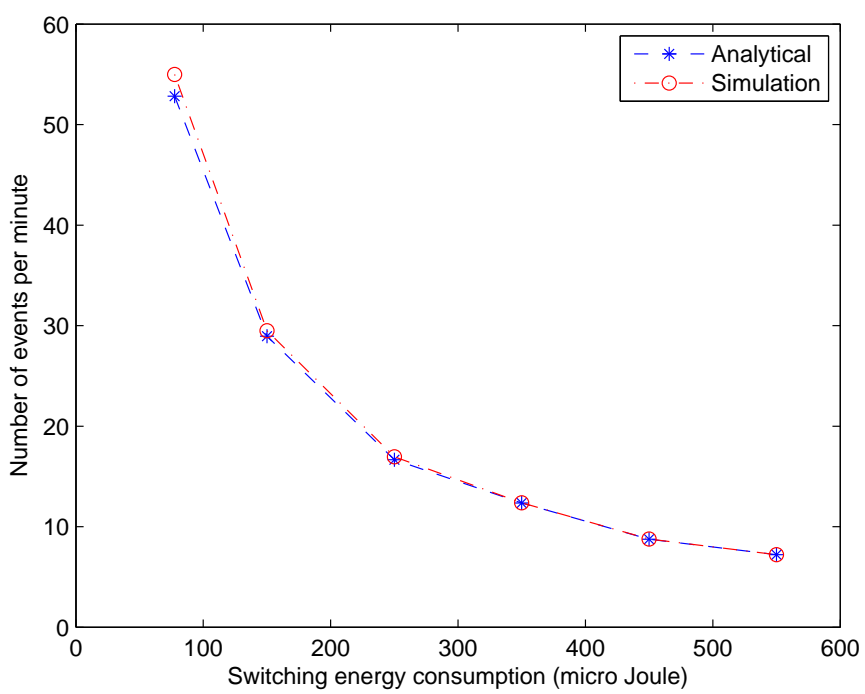

Figure 4-24: Critical event rate: Increasing the switching energy decreases the critical event rate. 


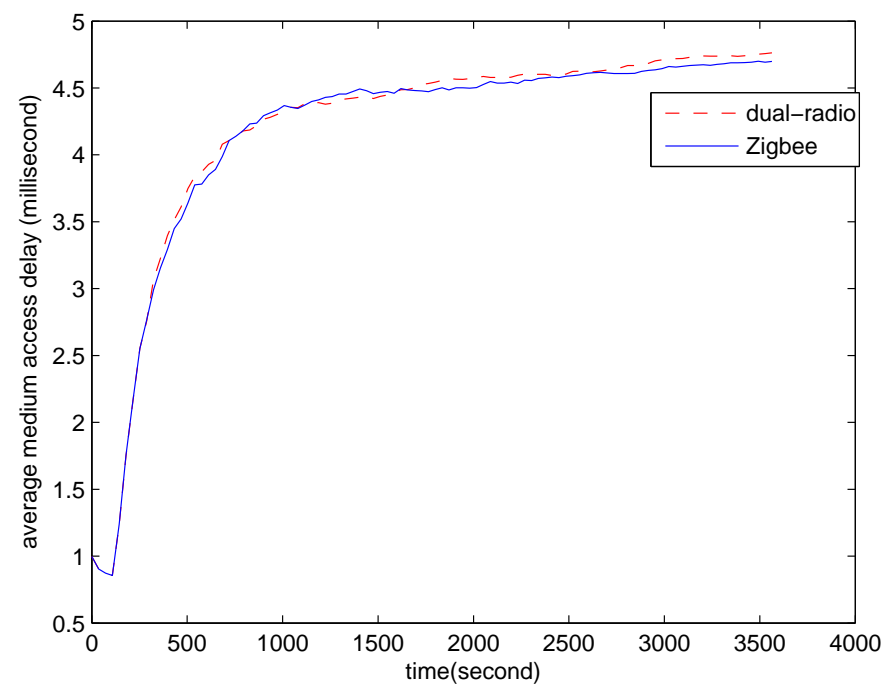

Figure 4-25: Medium access delay: The average medium access delay is approximately the same for two models. The $x$-axis represents the simulation time.

rate for this case results in more efficient communication.

\section{4-3-2 Delay}

In this part, we compare the delay of dual-radio network with Zigbee network. There is a trade-off between energy consumption and delay in wireless sensor networks. For instance, in a network which works based on SMAC we can decrease the energy consumption by increasing sleep periods. But this method increases delay in data transmission to a specific node which is mostly in sleep mode.

Figure 4-25 shows the average medium access delay for Zigbee and dual-radio node. As we see the average medium access delay is a bit larger for the dual-radio node in comparison to the Zigbee node. The Zigbee node sends the data by invoking the CSMA/CA process whenever it detects an event. But the dual-radio node does a Wake up Request and CTS transmission and reception before data transmission. So, the channel is not as free as the Zigbee network and it takes more time to access the channel for dual-radio node. As defined in OPNET, we considered the medium access delay as the time required to successful transmission of data and Wake up Request for Zigbee and dual-radio node respectively.

Figure 4-26 depicts the end-to-end delay. The end-to-end delay for dual-radio node is much less than the Zigbee node. When the dual-radio node sends data the channel is reserved for it's data transmission by the wake up radio. So, the probability of collision is decreased. The Zigbee node may need to retransmit the data packet, because it may collide with other nodes' packets. The channel is not reserved for the Zigbee node before the transmission starts, and the node only listens to the channel to check whether it is free before sending data. Note that the end-to-end 


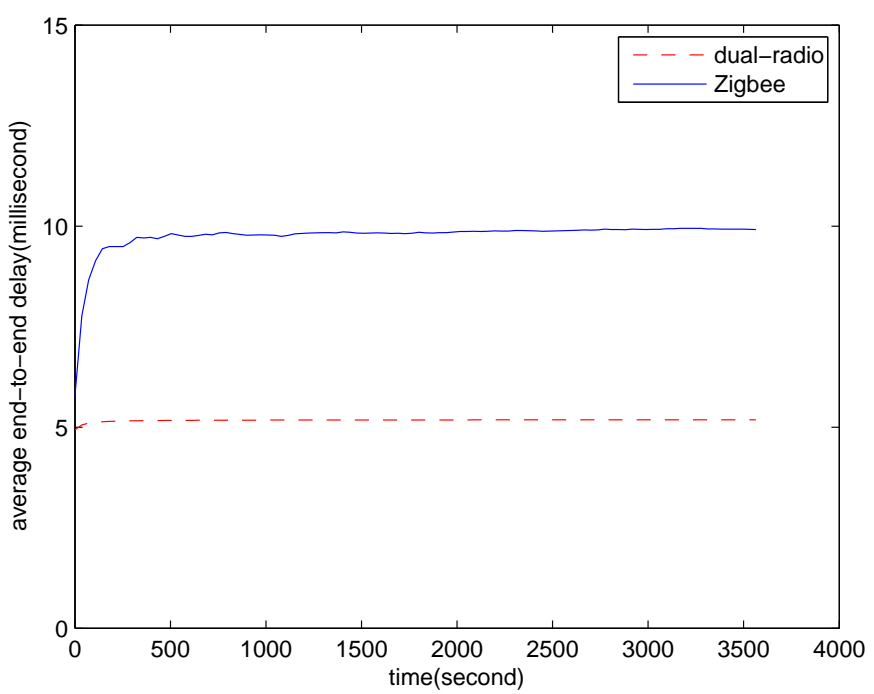

Figure 4-26: End-to-end delay: End-to-end delay for the dual-radio node is approximately half of the single-radio model. The $x$-axis represents the simulation time.

delay is defined as the time interval between the transmission and reception of a data packet.

\section{4-3-3 Zigbee Beacon-enabled Network}

In this section, we compare the energy consumption of the dual-radio node with Zigbee beacon-enabled node.

We have compared the dual-radio node model with non-beacon-enabled Zigbee network in the previous sections. The non-beacon-enabled Zigbee node does not have the ability to work based on a specific duty cycle and it should always be in the active state. Figure 4-27 represents the Zigbee beacon-enabled node model.

The node model is the same as the Zigbee non-beacon-enabled node with one difference: there is a separate battery module for computing the energy consumption. The details of the method of energy consumption calculation is different in this model from the previous node models. However, the working principles are the same.

In beacon-enabled mode, the beacon frames are periodically generated by the coordinator to identify the network, to synchronize devices that are associated with it, and to decribe the superframe structure [55].

The superframe structure is used by the coordinator to manage communication between the devices of the network. The superframe includes an active period and an optional inactive period. Each active period consists of a Contention Access Period (CAP) and an optional Contention Free Period (CFP). The CFP is activated when a request is sent from an end device to the coordinator. When the coordinator receives the request, it checks to see if there are sufficient resources. If it finds enough 


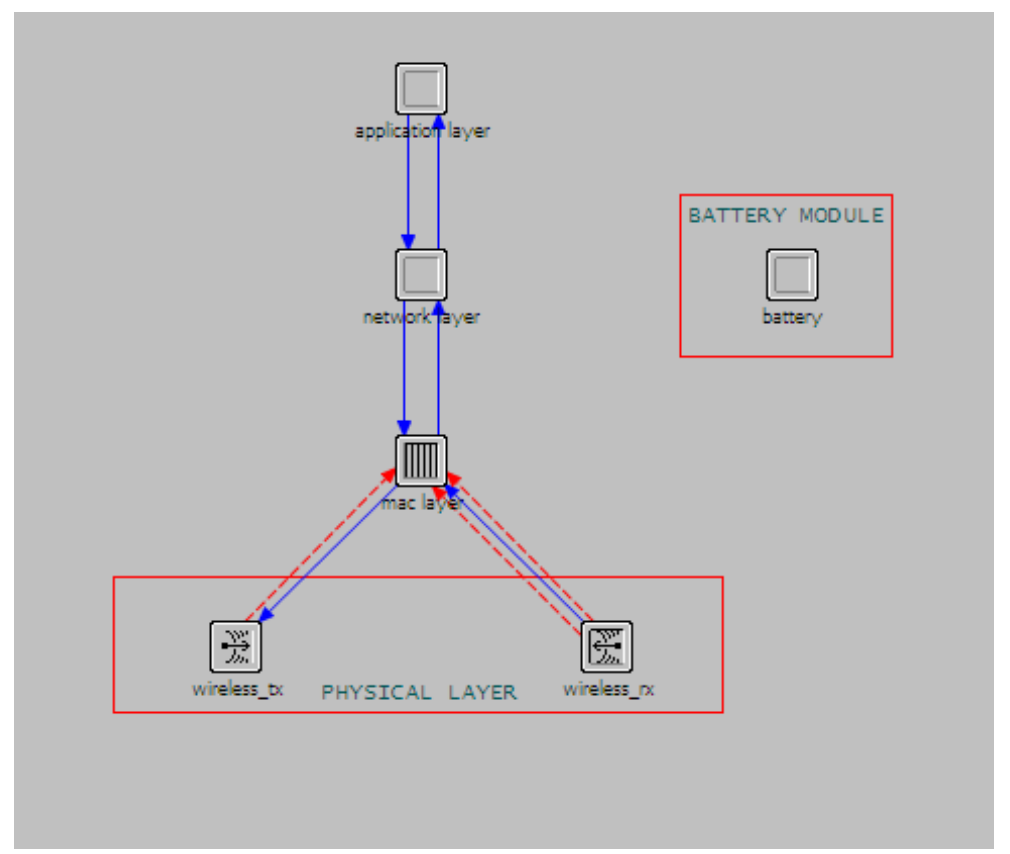

Figure 4-27: Zigbee beacon-enabled node model: A new battery module is added as the power source of the node. Application layer, network layer, data link layer, and physical layer are the four layers constituting the node model.

resources, it allocates the requested time slots. This requested group of time slots is named Guaranteed Time Slot (GTS). Figure 4-28 depicts the superframe structure.

Figure 4-29 shows the beacon-enabled MAC process model. It consists of seven states. The process model works based on slotted CSMA/CA for channel reservation. GTS-SLOT state defines the tasks of the node for the time that it is in contetion-free period.

In order to set the length of active and inactive intervals we should become familiar with the definition of some parameters of the Zigbee beacon-enabled mode. The duration of the active period (APR) is defined as:

$$
A P R=B S D \times 2^{S O}
$$

$S O$ is the Superframe Order which can be set as one of the attributes of the Zigbee node in the Zigbee beacon-enabled OPNET node model. $B S D$ is the duration of a base superframe.

The Beacon Interval (BI), which is the time between two consecutive beacons, is computed as:

$$
B I=B S D \times 2^{B O}
$$

$B O$ is the Beacon Order which again can be set as one of the attributes of the Zigbee node in the OPNET.

We define the duty cycle as the proportion of the active period to beacon interval. 


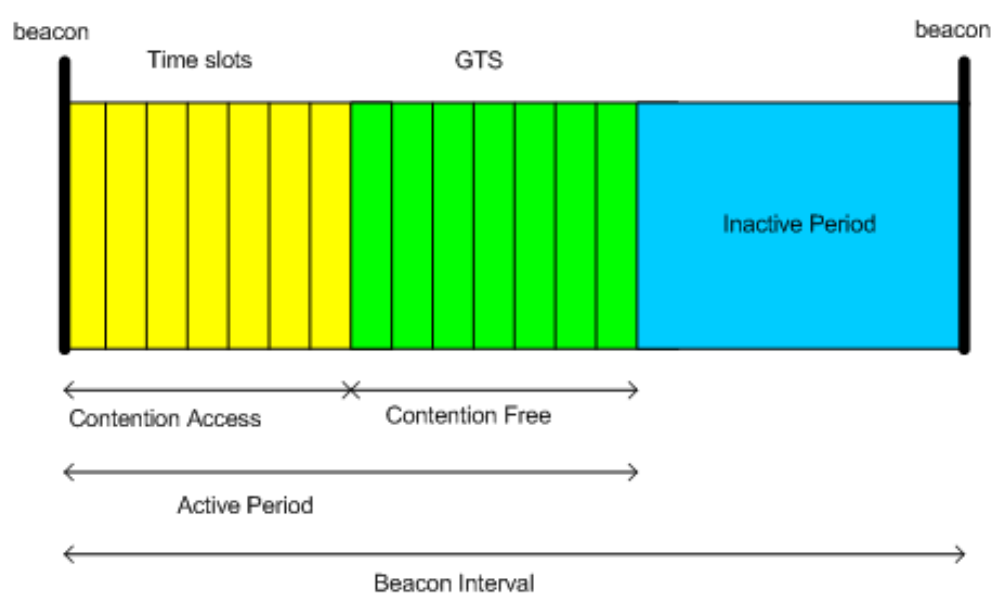

Figure 4-28: Superframe structure: Beacon interval is the time between two consecutive beacons. It consists of an active and an inactive period. The active period includes the contention free and contention access periods.

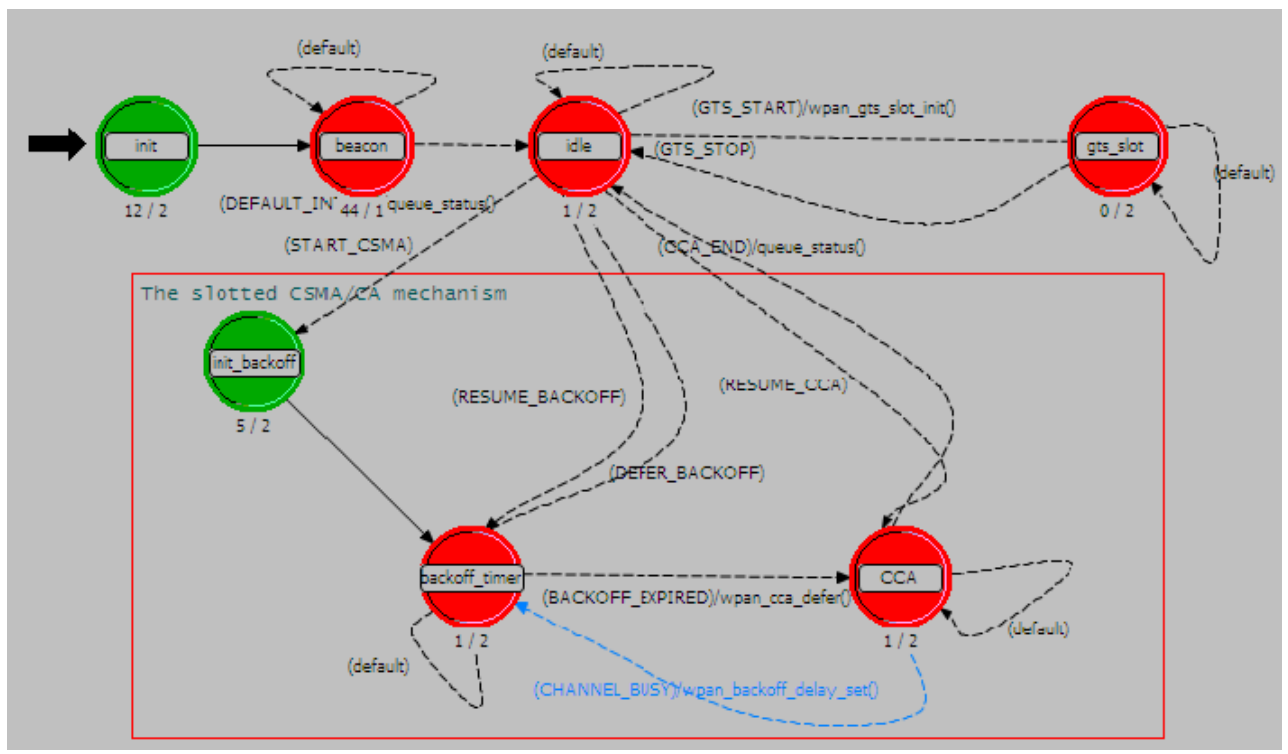

Figure 4-29: Zigbee beacon-enabled MAC process model: The process model defines contention access and contention free periods, and works based on slotted CSMA/CA mechanism. 


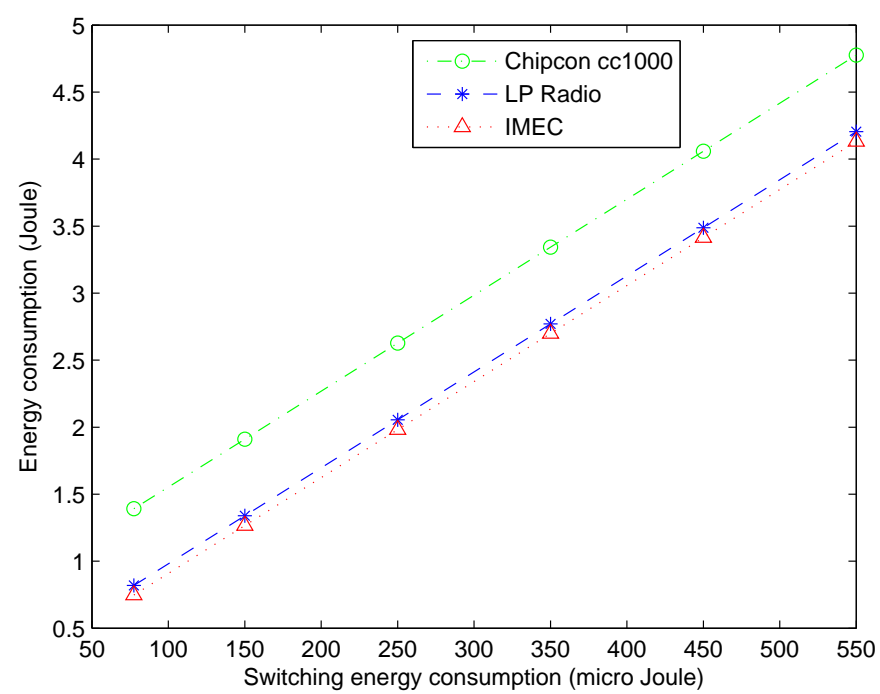

Figure 4-30: Node energy consumption for different Transition Energies: Since the mean event rate is one per second, increasing the switching energy has a large effect on the node's total energy consumption.

So, we can write:

$$
\text { dutycycle }=\frac{2^{S O}}{2^{B O}}=2^{S O-B O}
$$

For our simulation we set $B O$ to 8 and change $S O$ from 3 to 7 to have duty cycles from 3 to 50 percent.

Figure 4-30 shows the energy consumption of the three radios for different values of switching energy when the packet inter-arrival time has constant distribution with mean value 1 .

Figures 4-31, 4-32, and 4-33 show the energy consumption of Zigbe beacon-enabled end device for different duty cycles for Chipcon cc1000, LP Radio, and IMEC repectively.

For the case of Chipcon cc1000, the dual-radio performs much better than the Zigbee node. Only when the duyt cycle is $\frac{1}{32}$ and the switching energy is $300 \mu W$ or more, the Zigbee node consumes less energy than the dual-radio node. For all other assumed values of duty cycle and switching energy, dual-radio model has higher energy efficiency.

When LP Radio is used and the duty cycle is 50 percent, the dual-radio node performs better unless the switching energy is around $470 \mu J$ or more. This critical switching energy above which the Zigbee beacon-enabled node performs better than the dual-radio node is $180 \mu \mathrm{J}$ and $100 \mu \mathrm{J}$ for 25 and 12.5 percent duty cycles respectively. For lower duty cycles, the Zigbee node cosumes less energy than the dual-radio node even for the minimum switching energy that we assumed.

When IMEC is implemented in the nodes, the Zigbee node has higher energy efficiency for all assumed values of the switching energy. IMEC consumes low power 


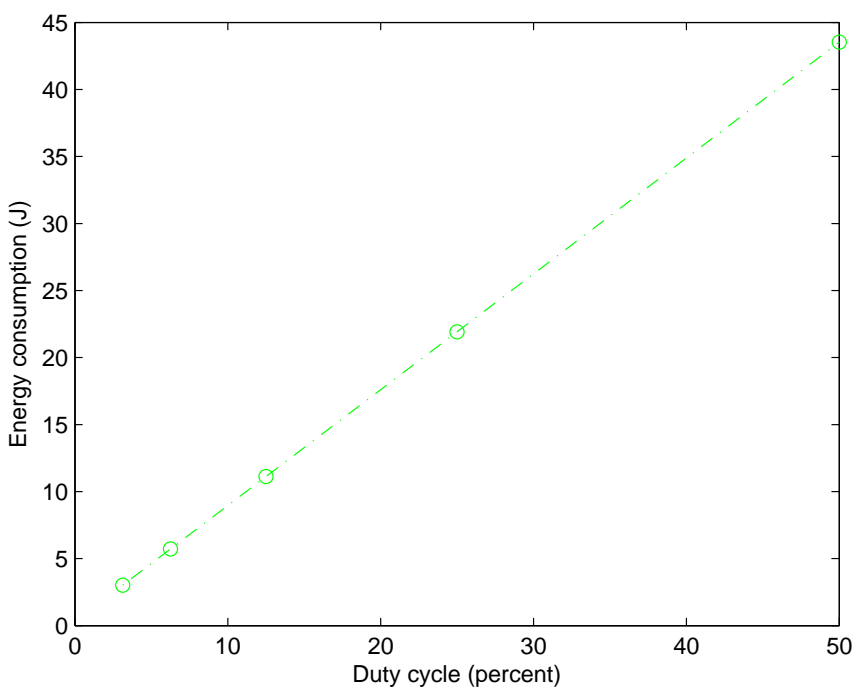

Figure 4-31: Node energy consumption for different duty cycles (Chipcon cc1000): Only when the duyt cycle is $\frac{1}{32}$ and the switching energy is $300 \mu W$ or more, dual-radio node performs worse than the single-radio Zigbee node.

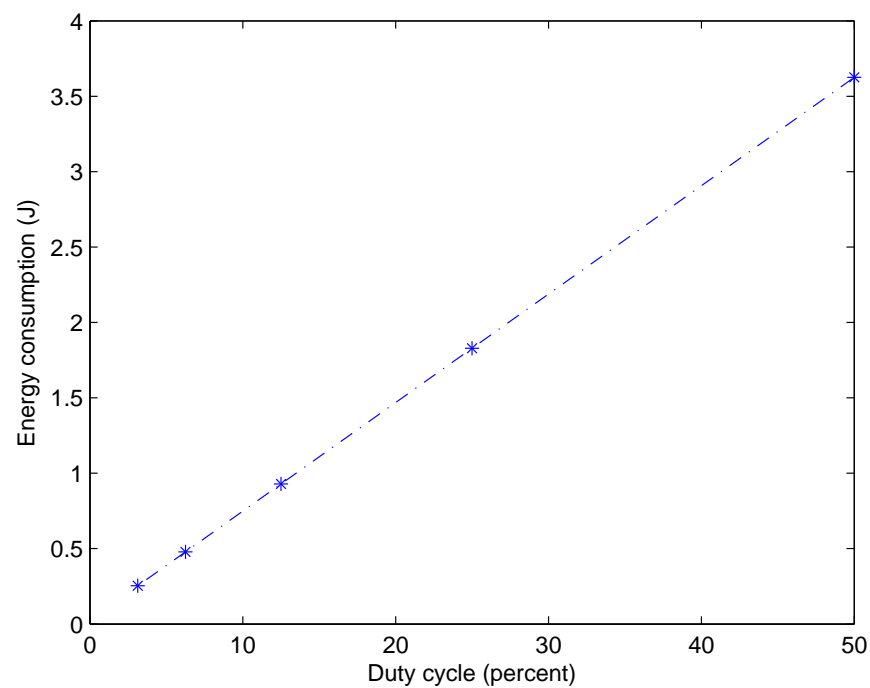

Figure 4-32: Node energy consumption for different duty cycles (LP Radio): For small duty cycles, $\frac{1}{32}$ and $\frac{1}{16}$, Zigbee node consumes less energy than dual-radio node. For larger duty cycles, there is a switching energy above which the dual-radio performs worse than Zigbee in terms of energy efficiency. This switching energy is $470 \mu J, 180 \mu J$, and $100 \mu J$ for 50,25 , and 12.5 per cent duty cycles respectively. 


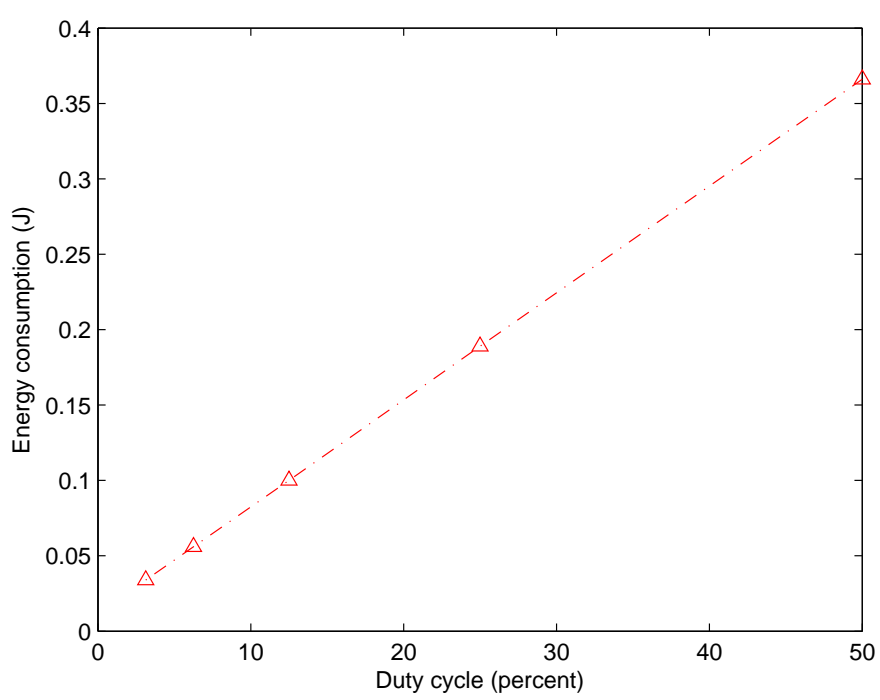

Figure 4-33: Node energy consumption for different duty cycles (IMEC): Due to low power consumption of the main radio model (IMEC), Zigbee node consumes less energy than the dual-radio node for all considered duty cycles.

consumption that is close to the power consumption of the wake up radio. Hence, when we consider it in the sleep mode for large proportions of the duty cycle, it's performance would be better than the dual-radio node.

\section{4-3-4 Discussion}

It is shown by simulation and analytically that the dual-radio node consumes less energy than Zigbee node in low event rates. Although our focus in this project is the energy issue, the efficiency of using the dual-radio node depends on some other parameters too. For instance, the duration of a transmission session is larger in a dual-radio network compared to a Zigbee network. For large event rates, this results in less data transmission. So, it may be not efficient to use dual-radio nodes in large event rates from this point of view even if the energy consumption is less for this model.

The overall improvement in power efficiency depends on many parameters. We briefly explain the most important ones.

\section{Radio Model}

First, the types of the main and wake up radio determine their power consumption during various states. If the main radio consumes much more power than the wake up radio during transmission, reception, and idle modes, the improvement of energy efficiecy caused by using a low-power wake up radio will be increased. As we saw in the results, the enery saved by using the wake up radio for the case that the main radio is Chipcon cc1000 is much more than the other two cases. 
The other characteristic of the main radio model that affects the consumed energy is it's data rate. The time that the node spends in transmission or reception of data is determined by the size of the packets and the radio data rate. The energy consumed during these states is the result of the multiplication of the time spent in each state, by the power consumption of the radio during them. So, lower data rate results in larger energy consumption. This is true for the wake up radio in case it does not work with the same power in all states.

The results depend on other characteristics of the wake up radio as well. For instance, using range-based or identity-based wake up radio has a large effect on the output. Range-based wake up radios consume extra energy for switching the main radio and waste energy in overhearing.

The mechanism used by the wake up radio to trigger the main radio into active or inactive mode affects the total energy consumption of the node. If the wake up radio extracts the energy required for this process from the radio signals, it will have higher energy efficiency. Otherwise, the energy consumption depends on the switching energy.

\section{Duty Cycle}

Second, using a protocol which works based on a duty cycle results in less energy consumption due to low power consumption of the radio during the sleep mode. We investigated the energy consumption of SMAC for different duty cycles analytically in the third chapter and Zigbee beacon-enabled node by simulation in the fourth chapter. Dependent on the radio model we use and the duty cycle, we see different results. For very low duty cycles, the node is in sleep mode most of the time and the dual-radio model consumes more energy especially when IMEC, which has a very low power consumption, is used. For large duty cycles depending on the main radio model, the dual-radio node has higher energy efficiency for small values of switching energy.

Note that the duty cycle scheme results in higher delay compared to dual-radio node since when a node has data to send for a specific target, it should wait until the active period of the destination. The dual-radio node solves this problem by using the wake up radio which is always active.

\section{Switching Energy}

Third, switching energy which is consumed to change the state of the main radio from active to inactive and vice versa, is another important factor affecting the energy efficiency of the dual-radio node. In very high event rates, the main radio is turned on and off a lot of times and this results in high energy consumption. Because of this point, in very high event rates the performance of the dual-radio node degrades and it would be better to use a single-radio model such as Zigbee non-beacon-enabled node. 


\section{Data Packet Size}

Data packet size affects the time that the main radio is transmitting and receiving data. Hence, it has an influence in the energy consumption of the main radio. Note that when the data rate of the main radio is very large, the effect of changing the data packet size would become negligible.

\section{4-3-5 Advantages and Disadvantages}

\section{Advantages}

Wake up radio results in significant energy consumption reduction in low event rate applications. The delay in data exchange and medium access is reasonable.

The idle listening time of the main radio which is a major source of energy waste is minimized. Nodes turn their main radio on only when they have to send or receive data by their main radio, and they monitor the channel by their low-power wake up radio.

Overhearing is decreased since the main radio is in sleep state by default. So, when two nodes transmit data packets to each other, the main radios of other neighbors is inactive and they do not receive the packet of other nodes. This way, the energy consumed to receive the messages of others is eliminated. This is not true for the range-based wake up radios which do not mention the address of the receiver in the wake up tone. They will receive the first packet sent by the main radio to identify the target node, and then switch their main radio to sleep mode.

Number of retransmissions due to collisions reduces. The channel is reserved by the wake up messages whose size is smaller than the data packets'. So, their collision probability is less than data packets' collision probability. If a collision happens, the always active wake up radio retransmits the wake up message and this does not extend the active time of the main radio which causes extra energy consumption.

Nodes send their packets to the neighbors. Thus, the overhead caused by routing is decreased.

\section{Disadvantages}

The use of an additional wake up radio requires extra implementation costs. Although the reduction in energy costs will be higher than this extra implementation costs, the funding needed to set up the network increases.

A transmission session lasts longer in a dual-radio network since there are additional wake up and CTS messages compared to the simple CSMA/CA process used for sending data in Zigbee. So, the delay is a bit larger in the dual-radio network especially in low event rates where the collision probability is really low.

The range of the wake up radio is less than the main radio. Hence, two nodes that are inside the main radio range of each other, but outside the wake up radio range, need additional messages and nodes to communicate to each other. 
Applications which have variable event rates may degrade the dual-radio system performance. In high event rates the energy efficiency of the dual-radio node degrades.

Since a transmission session takes longer time in the dual-radio node, the number of messages that can be transmitted during a specific time by the dual-radio node is less than the Zigbee node. So, when the event rate is very large, there may be packet losts due to full queues in dual-radio node.

\section{4-3-6 Wake-up Relay}

One problem with using a low-power wake up radio is its short range, which results in the need of a lot of additional hardware devices [5]. Moreover, this problem brings limitations for two nodes that are within each other's data transmission range, but out of wake up radio range [57].

An idea to solve this problem and optimize the system model is to change the MAC protocol so that the extra waking up of the main radios of the relaying nodes will be eliminated. It means that if a node wants to send data to a target which is within it's main radio range but outside the wake up radio range, sends a Wake up Request that contains the address of the final destination of the data. Then, the intermediate node which receives this message reads the destination address and checks if it is one of it's own neighbors. This intermediate node does not wake it's main radio up and just sends a Wake up Request by it's wake up radio to the target node. Once the target node receives the request, in case it is free, it sends the CTS message by it's wake up radio to the initial transmitter, using the intermediate node as a relay. Finally, the data transmission is done by the main radio. The Network Allocation Vectors included in Wake up Request and CTS messages should be set accoring to the time required for all these message exchanges to happen. The timers also should work based on the new model.

This method results in less energy consumption since some energy waste sources are decreased. First, the intermediate node does not consume extra energy for switching it's main radio since there is no need for it's main radio in this transmission session. Second, the number of transmissions and receptions done by the main radios is decreased. As we mentioned, the time that the main radio spends in active mode is a main source of energy consumption. By omitting the active time of the main radio of the intermediate node, and by decreasing the active time of the receiver and the transmitter of the data packet, the energy efficiency of the network increases.

The major problem of this scheme is the increased delay. The time that the channel is occupied by a specific transmission is increased since a transmission session consists of four wake up radio messages and a main radio data transmission and acknowledgement. While in the previous method a tranmission session includes two wake up radio messages and a main radio data transmission and acknowledgement. So, if a node which detects an event finds the channel busy, it should wait for more time to send it's data. 


\section{4-4 Dual-radio Cooperation}

In this section, we use the results of our work to propose a new rate-adaptive MAC protocol for dual-radio cooperation. The idea is to make use of the wake up radio in low event rates, and turn off the wake up radio and work with the main radio in high event rates.

For low event rates, the dual-radio node has higher energy efficiency compared to Zigbee node, and the use of wake up radio results in significant energy saving. For high event rates, dependent on the main radio model and switching energy, there is a critical point above which the performance of the dual-radio node degrades. It depends on many parameters to choose between the wake up radio or a singleradio node model for high traffic load applications. A great idea is to design a rate-adaptive MAC protocol which adapts to the event rate and changes it's MAC protocol when a specific threshold is reached. So, the dual-radio node is used and the MAC protocol should be changed in a way that for large event rates it does not make use of the wake up radio.

Implementing this idea in the MAC layer of the wireless radio is possible by adding a few instructions to it. First, we should compute the critical event rate of the wireless node based on the radio types and the switching energy. From this value, we compute the packet inter-arrival time below which the dual-radio system performs worse than the Zigbee protocol. Then, a condition is set so that if the packet interarrival time of a few consecutive incoming packets or events is below the computed threshold, the node takes it as the time to switch to the Zigbee MAC protocol.

The MAC protocol of the dual-radio node works based on the CSMA/CA process like the Zigbee protocol. The difference is that the dual-radio node invokes the CSMA/CA to send the Wake up Request by the wake up radio while the Zigbee MAC invokes CSMA/CA to send data by the main radio. So, when the threshold is reached, the dual-radio node should invoke the CSMA/CA to send data.

There are some other points that should be considered. The threshold is not reached for all the nodes at the same time. A node which expects to receive a Wake up Request and it's main radio is in sleep mode can not handle the data packet that is sent by a neighbor which has switched to Zigbee protocol. A method to solve this problem is to define a single bit in the Wake up Request message which shows the MAC protocol used for the next transmission. For instance, a zero is sent as the identifying bit when the event rate is low. Whenever a node intends to switch the MAC protocol, it sends one as the identifying bit in the last message sent by the wake up radio. The receiver of this message understands that it should turn it's main radio to active state and keep it on for the next data messages.

In the OPNET dual-radio node model (Figure 4-3), two modules, PASS and PASSWAKE, separate the MAC layer from the radios. These two modules can be used to discard the messages received by the wake up radio when the Zigbee protocol is working.

Dual-radio cooperation optimizes the operation of the wireless sensor network by taking advantage of the wake up radio in low event rates, and single-radio node in 
large event rates. In conclusion, by using dual-radio cooperation and the wake up relay idea, the energy efficiency of the wireless sensor network is maximized. 


\section{Chapter 5}

\section{Conclusion and Future Work}

\section{5-1 Conclusion}

Using a low-power wake up radio is a great idea to increase the energy efficiecy of wireless sensor networks. It achieves satisfactory communication delay while decreasing the node energy consumption significantly. Although the implementation cost is increased, the energy saving caused by minimizing the idle listening time of the main radio results in reduction of overall costs.

The idea of using the dual-radio node model focuses on the energy waste sources related to the data link layer. Reducing the energy dissipation in idle listening mode, decreasing overhearing and overhead, and reducing the number of retransmissions due to collisions are the goals of using a low-power wake up radio beside the node's main radio.

Idle listening is one of the major sources of energy waste in wireless sensor networks, especially in applications with low event rates. The main contribution of the wake up radio is minimizing the energy consumption during the idle listening. The lowpower wake up radio consumes much less power than the main radio model during the idle state. When an event is detected, and the node should send or receive data packets, the wake up radio triggers the main radio to active mode (from the default mode of sleeping) for data exchange. Therefore, the main radio is active only when data exchange occurs, and the method minimizes the active time of the main radio.

Identity-based wake up receivers minimize overhearing since a node is active only when it is involved in a transmission, and it does node receive the data packets destined for other nodes because it's main radio is in sleep mode by default. The communication overhead decreases, because each node wakes up it's neighbors. Number of collisions is reduced as channel is reserved by Wake up Request messages which are small in size. Before the main radio starts transmitting data packets, the channel is reserved by the wake up radio. Hence, the probability of collision of data 
packets is extremely low, and the main radio is not involved in channel reservation. This decreases the active time of the main radio.

Triggering the main radio into active and inactive states consumes energy. If the wake up radio uses the internal power source for this action, in large event rates the performance of the dual-radio model degrades. So, for large event rates, dependent on the radio models used and network parameters, the single-radio model may perform better than the dual-radio node. Another point to consider is that since a transmission session in dual-radio model consists of Wake up Request/Clear To Send exchange by the wake up radio, and data and Acknowledgement by the main radio, the time to transmit one packet is longer than the single-radio model. Another source of delay is the time required to trigger the main radio. Thus, the maximum number of packets that can be sent in the unit of time is less for the dual-radio model. Considering all these, it may be better to use the single-radio model for large event rates. Critical event rate is defined as the rate above which single-radio model performs better than the dual-radio node.

In this project, we have compared the dual-radio model performance with singleradio node model which works based on CSMA/CA protocol. An analytical model for computing the wireless node's energy consumption is described. We have tested the energy efficiency for three different radio types as the main radio, and confirmed the analytical model with the simulation results. The results show that when a radio with much higher power consumption than the wake up radio is used as the main radio model, there is huge energy saving by the dual-radio model. For radios with lower power consumption, the difference is smaller, and dependent on the switching energy, the single-radio may consume less energy in large event rates. However, for low event rates, energy efficiency of dual-radio model is larger. We investigated the effect of switching energy on the critical event rate. We also showed that the communication delay of the dual-radio model is satisfying.

Dual-radio cooperation takes the benefits of the wake up radio in low event rates and the critical event rate concept into account, and proposes a rate-adaptive model to optimize the performance of the wireless sensor network. The idea is to use nodes equipped with a low-power wake up radio in the wireless network. The dualradio node model works based on the Wake up Request/Clear To Send exchange mechanism by the wake up radio, and data and Acknowledgement exchange by the main radio in low event rates; For event rates larger than the critical threshold, nodes use their main radio for data exchange and do not use their wake up radio. In fact, they work as a single-radio node which invokes CSMA/CA process to send data. This method results in high energy efficiency for both large and low event rates.

\section{5-2 Future Work}

Future work will be implementing and testing the rate-adaptive MAC protocol to optimize the designed model. Dual-radio cooperation is a novel idea to maximize the energy efficiency of wireless sensor networks. It makes use of the wake up radio 
in low event rates and switches to the single-radio operation in large event rates. Implementing the MAC protocol of the dual-radio cooperation by considering the critical event rate concept, optimizes the performance of the huge wireless sensor networks.

The problem with this idea is the communication delay. Using dual-radio cooperation may result in extra delay around the critical event rate. Finding solutions to optimize the energy/delay trade-off and designing a new MAC protocol for dualradio cooperation to decrease the communication delay for the applications in which minimizing delay is the prominent concern will be the future research topic. 


\section{Bibliography}

[1] I. Demirkol, C. Ersoy, and E. Onur, "Wake-up receivers for wireless sensor networks: benefits and challenges," Wireless Communications, IEEE, vol. 16, no. 4 , pp. $88-96,2009$.

[2] E.-Y. Lin, J. Rabaey, and A. Wolisz, "Power-efficient rendez-vous schemes for dense wireless sensor networks," in Communications, 2004 IEEE International Conference on, vol. 7, 2004, pp. 3769 - 3776 Vol.7.

[3] C. Schurgers, V. Tsiatsis, S. Ganeriwal, and M. Srivastava, "Optimizing sensor networks in the energy-latency-density design space," Mobile Computing, IEEE Transactions on, vol. 1, pp. 70 - 80, 2002.

[4] J. Mathews, M. Barnes, A. Young, and D. K. Arvind, "Low power wake-up in wireless sensor networks using free space optical communications," in Proceedings of the 2010 Fourth International Conference on Sensor Technologies and Applications, ser. SENSORCOMM '10. Washington, DC, USA: IEEE Computer Society, 2010, pp. 256-261. [Online]. Available: http://dx.doi.org/10.1109/SENSORCOMM.2010.47

[5] E. Shih, P. Bahl, and M. J. Sinclair, "Wake on wireless: An event driven energy saving strategy for battery operated devices," in Proceedings of the 8th annual international conference on Mobile computing and networking, ser. MobiCom '02. New York, NY, USA: ACM, 2002, pp. 160-171. [Online]. Available: http://doi.acm.org/10.1145/570645.570666

[6] L. Huang, M. Ashouei, F. Yazicioglu, J. Penders, R. Vullers, G. Dolmans, P. Merken, J. Huisken, H. de Groot, C. Van Hoof, and B. Gyselinckx, "Ultralow power sensor design for wireless body area networks: Challenges, potential solutions, and applications," JDCTA: International Journal of Digital Content Technology and its Applications, vol. 3, no. 3, pp. 136-148, 2009.

[7] R. Jurdak, A. Ruzzelli, and G. O'Hare, "Multi-hop rfid wake-up radio: Design, evaluation and energy tradeoffs," in Computer Communications and Networks, 
2008. ICCCN '08. Proceedings of 17th International Conference on, aug. 2008, pp. $1-8$.

[8] L. Song and D. Hatzinakos, "A cross-layer architecture of wireless sensor networks for target tracking," Networking, IEEE/ACM Transactions on, vol. 15, pp. $145-158$, feb. 2007.

[9] Y. Zhang, G. Dolmans, L. Huang, X. Huang, M. Lont, D. Milosevic, and P. Baltus, "Imec narrowband mac proposal," Holst Centre/IMEC-NL, Eindhoven University of Technology, Tech. Rep., 052009.

[10] C. Guo, L. C. Zhong, and J. Rabaey, "Low power distributed mac for ad hoc sensor radio networks," in Global Telecommunications Conference, 2001. GLOBECOM '01. IEEE, vol. 5, 2001, pp. $2944-2948$ vol.5.

[11] T. Walteyne, A. Bachir, M. Dohler, D. Barthe, and I. Auge-Blum, "1-hopmac: An energy-efficient mac protocol for avoiding 1 -hop neighborhood knowledge," in Sensor and Ad Hoc Communications and Networks, 2006. SECON '06. 2006 3rd Annual IEEE Communications Society on, vol. 2, sept. 2006, pp. $639-644$.

[12] A. Bachir, M. Dohler, T. Watteyne, and K. Leung, "Mac essentials for wireless sensor networks," Communications Surveys Tutorials, IEEE, vol. 12, pp. 222 $-248,2010$.

[13] Y. Tay, K. Jamieson, and H. Balakrishnan, "Collision-minimizing csma and its applications to wireless sensor networks," Selected Areas in Communications, IEEE Journal on, vol. 22, no. 6, pp. 1048 - 1057, aug. 2004.

[14] L. Kleinrock and F. Tobagi, "Packet switching in radio channels: Part i-carrier sense multiple-access modes and their throughput-delay characteristics," Communications, IEEE Transactions on, vol. 23, no. 12, pp. 1400 - 1416, dec 1975.

[15] F. Bouabdallah, N. Bouabdallah, and R. Boutaba, "On balancing energy consumption in wireless sensor networks," Vehicular Technology, IEEE Transactions on, vol. 58, no. 6, pp. 2909 -2924, july 2009.

[16] P. Karn, "Maca-a new channel access method for packet radio," in $A R R L C R R L$ Amateur radio 9th computer networking conference, vol. 140, 1990, pp. 134-140.

[17] A. Woo and D. E. Culler, "A transmission control scheme for media access in sensor networks," in Proceedings of the 7th annual international conference on Mobile computing and networking, ser. MobiCom '01. New York, NY, USA: ACM, 2001, pp. 221-235. [Online]. Available: http://doi.acm.org/10.1145/381677.381699

[18] C. S. Singh, S. Raghavendra, "Pasmas - power aware multi access protocol with signaling for adhoc networks," in ACM SIGCOMM Computer Communication Review, vol. 28, 1998, pp. 1048-1057.

[19] L. Pister, K. Doherty, "Tsmp: Time synchronized mesh protocol," in Parallel and Distributed Computing Systems (PDCS), 2008. 
[20] S. Ergen and P. Varaiya, "Pedamacs: power efficient and delay aware medium access protocol for sensor networks," Mobile Computing, IEEE Transactions on, vol. 5, no. 7, pp. $920-930$, july 2006 .

[21] M. Ringwald and K. Romer, "Bitmac: a deterministic, collision-free, and robust mac protocol for sensor networks," in Wireless Sensor Networks, 2005. Proceeedings of the Second European Workshop on, jan.-2 feb. 2005, pp. 57 69.

[22] M. Brownfield, K. Mehrjoo, A. Fayez, and I. Davis, N.J., "Wireless sensor network energy-adaptive mac protocol," in Consumer Communications and Networking Conference, 2006. CCNC 2006. 3rd IEEE, vol. 2, jan. 2006, pp. $778-782$.

[23] K. Sohrabi, J. Gao, V. Ailawadhi, and G. Pottie, "Protocols for selforganization of a wireless sensor network," Personal Communications, IEEE, vol. 7 , no. 5 , pp. $16-27$, oct 2000 .

[24] V. Rajendran, K. Obraczka, and J. Garcia-Luna-Aceves, "Energy-efficient, collision-free medium access control for wireless sensor networks." ACM Press, 2003, pp. 181-192.

[25] V. Rajendran, J. Garcia-Luna-Aveces, and K. Obraczka, "Energy-efficient, application-aware medium access for sensor networks," in Mobile Adhoc and Sensor Systems Conference, 2005. IEEE International Conference on, nov. 2005, pp. 8 pp. -630 .

[26] L. van Hoesel, T. Nieberg, H. Kip, and P. Havinga, "Advantages of a tdma based, energy-efficient, self-organizing mac protocol for wsns," in Vehicular Technology Conference, 2004. VTC 2004-Spring. 2004 IEEE 59th, vol. 3, may 2004, pp. 1598 - 1602 Vol.3.

[27] I. Cheong, P. Oppermann, "An energy-efficient positioning-enabled mac protocol(pmac) for uwb sensor networks," in EURASIP IST, Dresden, Germany, 2005.

[28] G. Pei and C. Chien, "Low power tdma in large wireless sensor networks," in Military Communications Conference, 2001. MILCOM 2001. Communications for Network-Centric Operations: Creating the Information Force. IEEE, vol. 1, 2001, pp. $347-351$ vol.1.

[29] J. Li and G. Lazarou, "A bit-map-assisted energy-efficient mac scheme for wireless sensor networks," in Information Processing in Sensor Networks, 2004. IPSN 2004. Third International Symposium on, april 2004, pp. 55 - 60.

[30] M. Ali, T. Suleman, and Z. Uzmi, "Mmac: a mobility-adaptive, collision-free mac protocol for wireless sensor networks," in Performance, Computing, and Communications Conference, 2005. IPCCC 2005. 24th IEEE International, april 2005, pp. $401-407$. 
[31] W. Lee, A. Datta, and R. Cardell-Oliver, "Fleximac: A flexible tdma-based mac protocol for fault-tolerant and energy-efficient wireless sensor networks," in Networks, 2006. ICON '06. 14th IEEE International Conference on, vol. 2, sept. 2006 , pp. $1-6$.

[32] T. Zheng, S. Radhakrishnan, and V. Sarangan, "Pmac: an adaptive energyefficient mac protocol for wireless sensor networks," in Parallel and Distributed Processing Symposium, 2005. Proceedings. 19th IEEE International, april 2005.

[33] H. Cao, K. Parker, and A. Arora, "O-mac: A receiver centric power management protocol," in Network Protocols, 2006. ICNP '06. Proceedings of the 2006 14th IEEE International Conference on, nov. 2006, pp. $311-320$.

[34] Y. Kim, H. Shin, and H. Cha, "Y-mac: An energy-efficient multi-channel mac protocol for dense wireless sensor networks," in Proceedings of the 7th international conference on Information processing in sensor networks. Washington, DC, USA: IEEE Computer Society, 2008, pp. 53-63. [Online]. Available: http://dx.doi.org/10.1109/IPSN.2008.27

[35] J. E. D. Ye, W. Heidemann, "An energy-efficient mac protocol for wireless sensor networks," in Annual Joint Conference of the Computer and Communication Societies (INFOCOM), 2002, pp. 1567-1576.

[36] K. Van Dam, T. Langendoen, "An adaptive energy-efficient mac protocol for wireless sensor networks," in Proceedings of the 1st international conference on Embedded networked sensor systems, 2003, pp. 171-180.

[37] S.-H. Yang, H.-W. Tseng, E.-K. Wu, and G.-H. Chen, "Utilization based duty cycle tuning mac protocol for wireless sensor networks," in Global Telecommunications Conference, 2005. GLOBECOM '05. IEEE, vol. 6, dec. 2005, pp. 5 pp. -3262 .

[38] A. Warrier, J. Min, and I. Rhee, "Z-mac: a hybrid mac for wireless sensor networks." ACM Press, 2005, pp. 90-101.

[39] S. H. Lee, J. H. Park, and L. Choi, "Amac: Traffic-adaptive sensor network mac protocol through variable duty-cycle operations," in Communications, $200 \%$. ICC 'O\%. IEEE International Conference on, june 2007, pp. 3259 -3264.

[40] V. Jain, R. Biswas, and D. P. Agrawal, "Energy-efficient and reliable medium access in sensor networks," in World of Wireless, Mobile and Multimedia Networks, 200\%. WoWMoM 200\%. IEEE International Symposium on a, june 2007, pp. $1-8$.

[41] G. Weber, V. Vickery, "Smart sensor networks: Technologies and applications for green growth," OECD, Tech. Rep., 2009.

[42] C.-Y. Chong and S. Kumar, "Sensor networks: evolution, opportunities, and challenges," Proceedings of the IEEE, vol. 91, no. 8, pp. 1247 - 1256, aug. 2003. 
[43] I. Akyildiz, W. Su, Y. Sankarasubramaniam, and E. Cayirci, "A survey on sensor networks," Communications Magazine, IEEE, vol. 40, no. 8, pp. $102-$ 114, aug 2002.

[44] "Intelligent street lighting," Jennic Ltd, Sheffield UK, Tech. Rep., 042009.

[45] S. Le-Huy, P. Roy, "Low-power wake-up radio for wireless sensor networks," Mobile Networks and Applications - MONET, vol. 15, pp. 226-236, 2010.

[46] M. Hefeeda and M. Bagheri, "Wireless sensor networks for early detection of forest fires," in Mobile Adhoc and Sensor Systems, 200\%. MASS 200\%. IEEE Internatonal Conference on, oct. 2007, pp. $1-6$.

[47] L. Yu, N. Wang, and X. Meng, "Real-time forest fire detection with wireless sensor networks," in Wireless Communications, Networking and Mobile Computing, 2005. Proceedings. 2005 International Conference on, vol. 2, sept. 2005, pp. $1214-1217$.

[48] A.-A. Al-Habashneh, M. Ahmed, and T. Husain, "Adaptive mac protocols for forest fire detection using wireless sensor networks," in Electrical and Computer Engineering, 2009. CCECE '09. Canadian Conference on, may 2009, pp. 329 -333 .

[49] K. W. van der Doorn, B. and K. Langendoen, "A prototype low-cost wakeup radio for the $868 \mathrm{mhz}$ band," Int. J. Sensor Networks, vol. 5, no. 1, pp. 22-32, 2009 .

[50] H. wei Tseng, S.-H. Yang, P.-Y. Chuang, H.-K. Wu, and G.-H. Chen, "An energy consumption analytic model for a wireless sensor mac protocol," in Vehicular Technology Conference, 2004. VTC2004-Fall. 2004 IEEE 60th, vol. 6, sept. 2004, pp. 4533 - 4537 Vol. 6.

[51] D. M. P. Ansari, J. Pankin, "Radio-triggered wake-ups with addressing capabilities for extremely low power sensor network applications," International Journal of Wireless Information Networks, vol. 16, pp. 118-130, 2008.

[52] S. Drago, F. Sebastiano, L. Breems, D. Leenaerts, K. Makinwa, and B. Nauta, "Impulse-based scheme for crystal-less ulp radios," Circuits and Systems I: Regular Papers, IEEE Transactions on, vol. 56, no. 5, pp. 1041 -1052, may 2009.

[53] L. Z. Y. Dolmans, G. Huang, "Preliminary imec proposal," Holst Centre/IMEC-NL, Tech. Rep., 032009.

[54] [Online]. Available: www.opnet.com

[55] A. Jurcik, P. Koubaa, "The ieee 802.15.4 opnet simulation model: Reference guide v2.0," Polytechnic Institute of Porto (ISEP-IPP), Tech. Rep., 2007.

[56] L. Gu and J. A. Stankovic, "Radio-triggered wake-up for wireless sensor networks," Real-Time Syst., vol. 29, pp. 157-182, March 2005. [Online]. Available: http://portal.acm.org/citation.cfm?id=1063921.1063946 
[57] N. Yang, X. Vaidya, "A wakeup scheme for sensor networks: Achieving balance between energy saving and end-to-end delay," in Proceedings of the 10th IEEE Real-Time and Embedded Technology and Applications Symposium. Washington, DC, USA: IEEE Computer Society, 2004, pp. 19-. [Online]. Available: http://portal.acm.org/citation.cfm?id=998685.1006949 